\title{
Agent-Based Models of the Tumor Microenvironment: Predicting Flow-Mediated Invasion and Cancer Viability in Response to Interstitial Flow, Chemotherapy, and Stromal Cell Density
}

\author{
Daniel Kendrick Logsdon
}

A thesis submitted to the faculty at the University of Virginia in partial fulfillment of the requirements for the degree of Master of Science (M.S.) in Biomedical Engineering in the School of Engineering and Applied Sciences.

University of Virginia

$04 / 10 / 2017$ 


\section{Table of Contents}

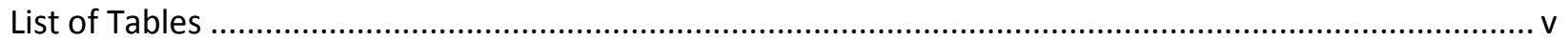

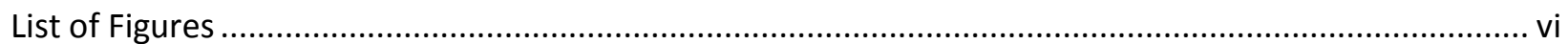

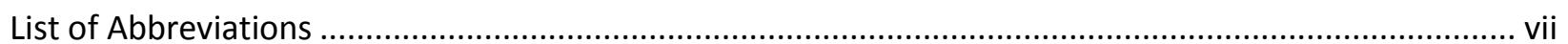

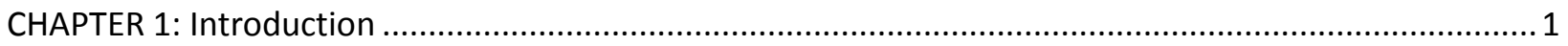

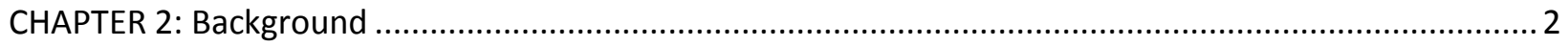

2.1 The Tumor Microenvironment …...........................................................................................

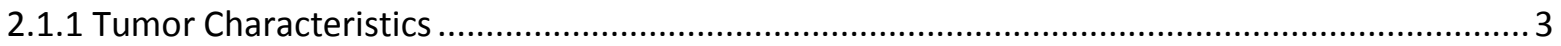

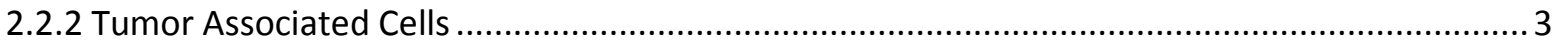

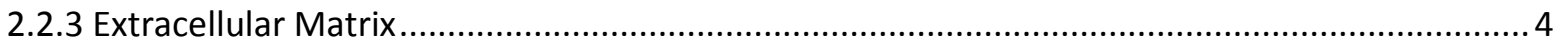

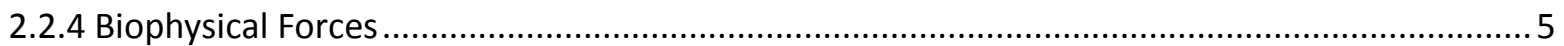

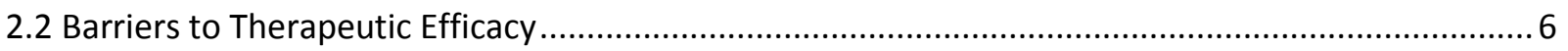

2.2.1 Physical Barriers to Therapeutic Efficacy ............................................................................. 6

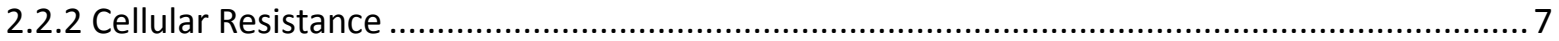

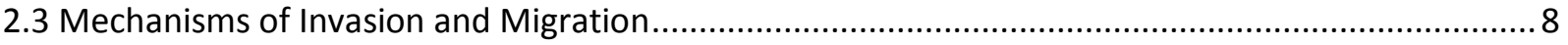

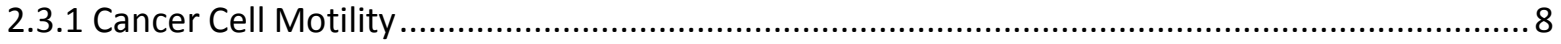

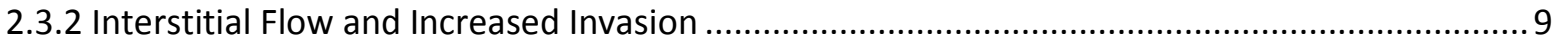

2.4 3D In Vitro Models of the Tumor Microenvironment .................................................................. 10

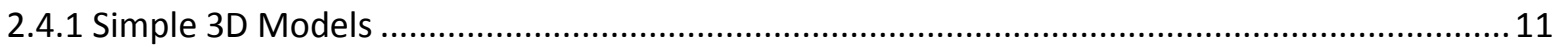

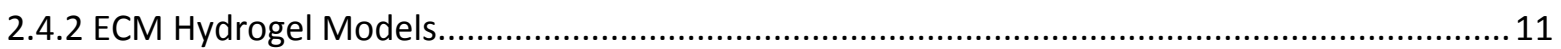

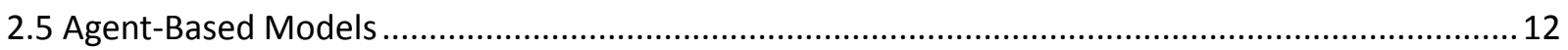

2.5.1 An Introduction to Agent-Based Models ........................................................................... 12

2.5.2 Agent-Based Models of Cellular Motility …............................................................................... 13

2.5.3 Agent-Based Models of Pharmacometrics............................................................................ 14

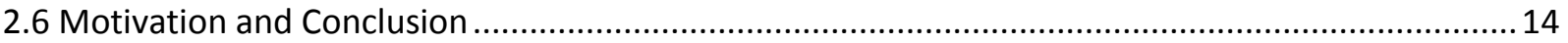

CHAPTER 3: An Agent-Based Model of Cancer Cell Invasion and Migration ........................................... 16

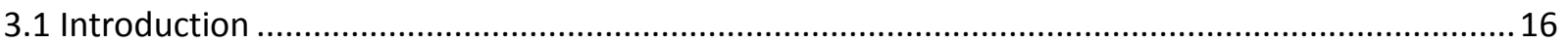

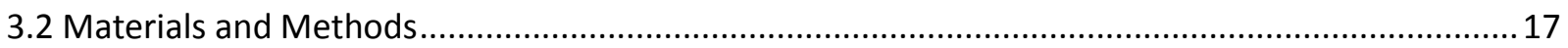

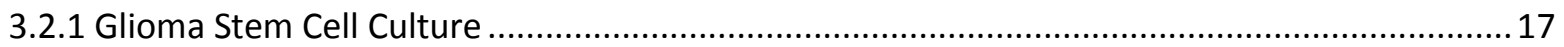

3.2.2 Three-Dimensonal (3D) In Vitro Interstitial Flow Model ...................................................... 17

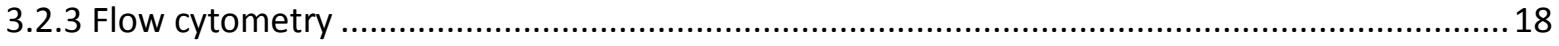

3.2.4 Agent-Based Cell Migration Model Construction .................................................................. 19

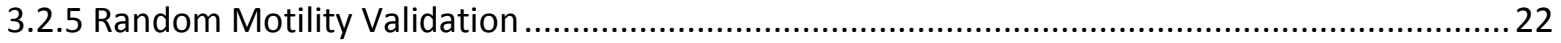




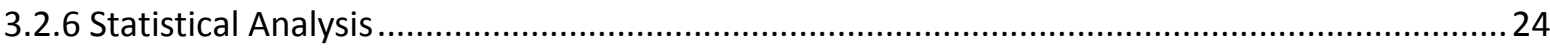

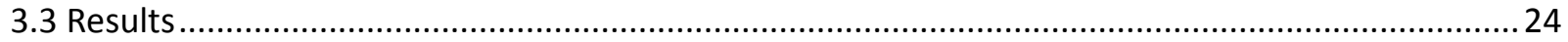

3.3.1 Simulated random motility is representative of experimental cellular motility in the absence

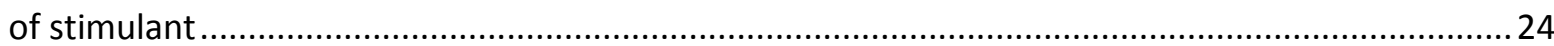

3.3.2 Mechanotransduction and autologous chemotaxis mediate flow responsive invasion ........... 26

3.3.3 Two subpopulations account for increased motility in the presence of interstitial flow.......... 28

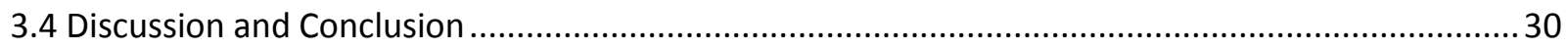

3.4.1 Subpopulation protein expression mediates flow responsive invasion ...................................30

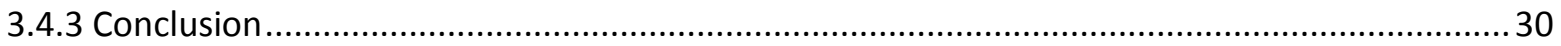

CHAPTER 4: An Agent-Based Model of the Tumor Border Transitional Microenvironment......................32

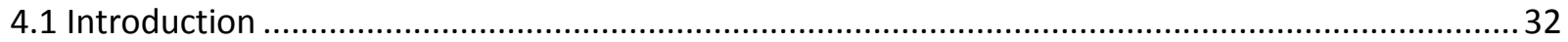

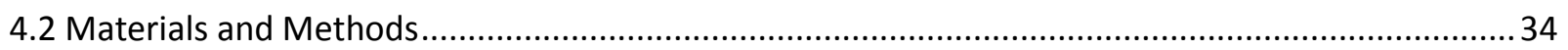

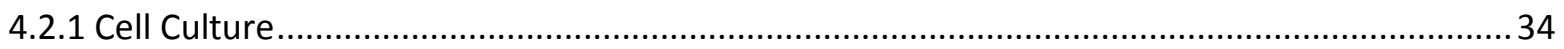

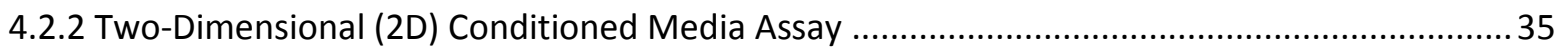

4.2.3 Two-Dimensional (2D) Hanging Well Coculture Assay ........................................................ 35

4.2.4 Two-Dimensional (2D) Ratiometric Coculture Assay .............................................................. 35

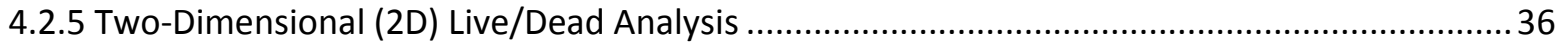

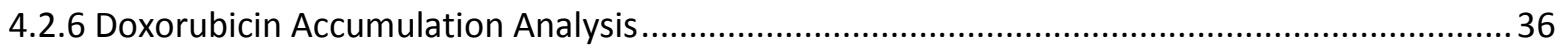

4.2.7 Three-Dimensional (3D) Homogenous In vitro Interstitial Flow Model .................................. 37

4.2.8 Three Dimensional (3D) In vitro Tumor to Stroma Transition Hydrogel Model ........................37

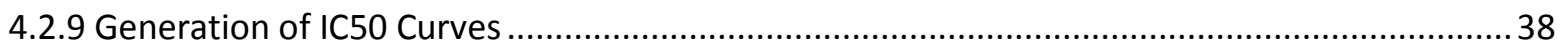

4.2.10 Development of Concentration Gradients using Comsol ...................................................39

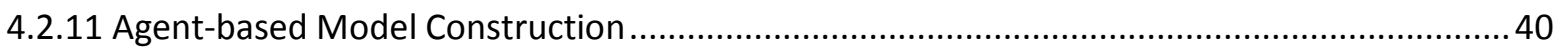

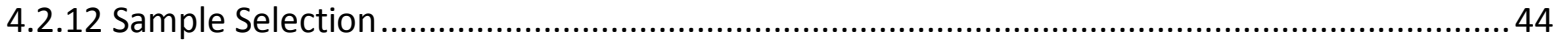

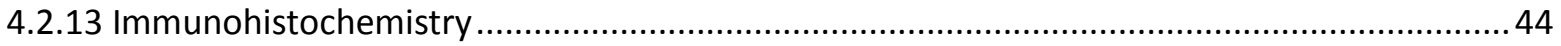

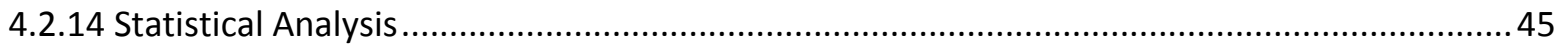

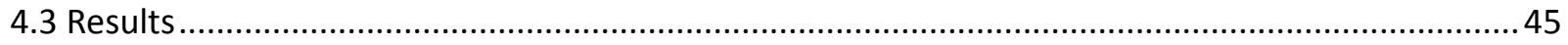

4.3.1 Fibroblasts reduce cancer cell death in response to doxorubicin and lowered levels of

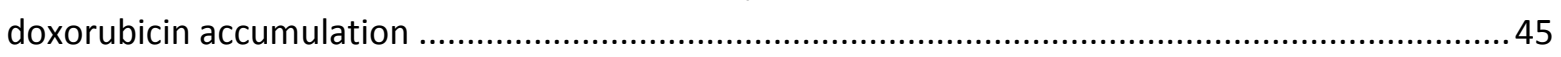

4.3.2 Cancer cell response to doxorubicin is dependent on the ratio of tumor cells:fibroblasts ...... 47

4.3.3 Ratiometric effects are conserved when cells are cultured in a breast-mimetic 3D

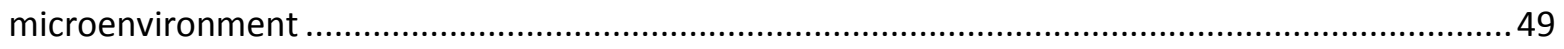

4.3.4 Gradients of chemotherapy form across invading edges of tumors .................................... 51 
4.3.5 High tumor cells to fibroblasts ratios reduce cancer cell response in an Agent-Based Model of

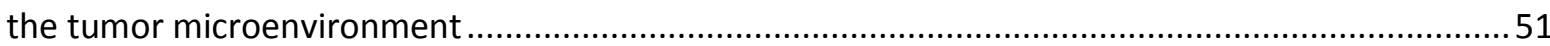

4.3.6 Development of an in silico tumor to stromal transition (TST) model ABM shows response across the invasive edge of tumors

4.3.7 A corresponding in vitro model of the invasive edge indicates similar region specific cancer cell response

4.3.8 The interaction of transport properties with cellular interactions in the ABM shows the importance of tissue- level changes in cancer cell response to chemotherapy...............................56

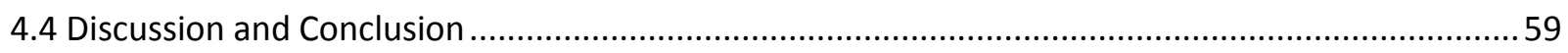

4.4.1 Mechanisms behind fibroblast induced chemotherapy resistance .........................................59

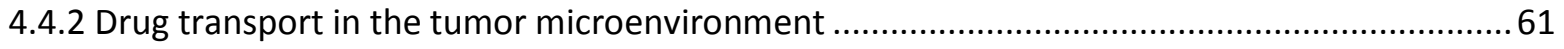

4.4.3 Implications of our models to therapeutic efficacy and clinical response ..............................62 62

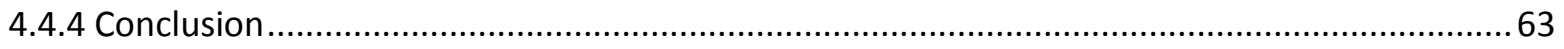

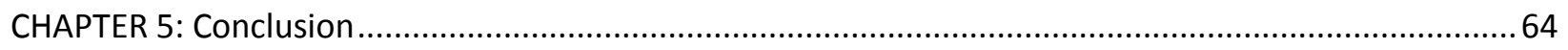

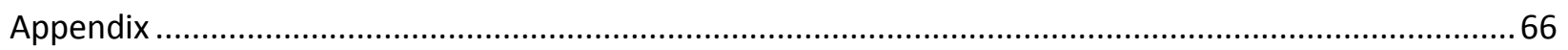

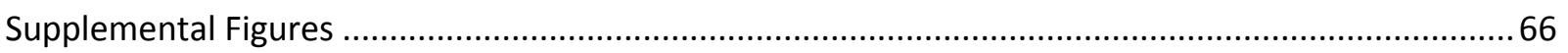

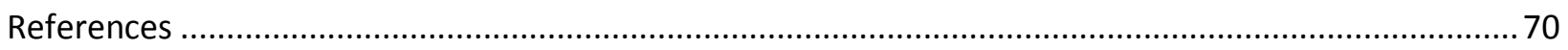




\section{List of Tables}

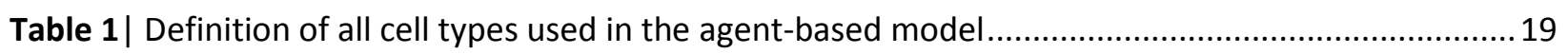

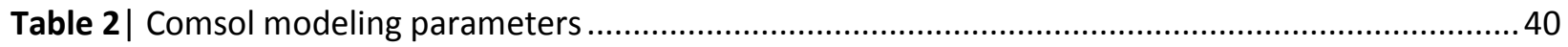

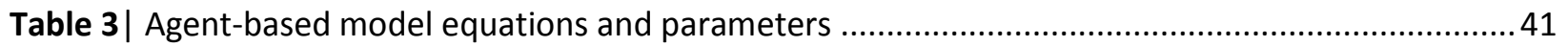

Table 4| Antibodies used for immunohistochemical staining............................................................. 44 


\section{List of Figures}

Figure 1|Components of the tumor microenvironment.

Figure 2| Proposed dominant mechanisms for increased invasion with interstitial flow. ....................... 10

Figure 3| Experimental setup of in vitro hydrogels for invasion studies.............................................. 18

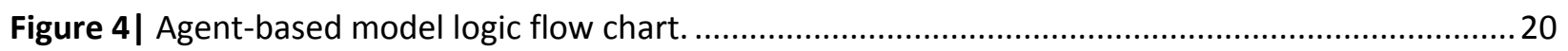

Figure 5| Specific ABM methods flow chart. ................................................................................. 21

Figure 6| Replication of supplemental Figure 5 from Emonet et al. for validation of agent model...........25

Figure 7| Random motility validation with experimental data.........................................................26

Figure 8| Development of an agent-based model predicts flow-stimulated invasion response to blocking

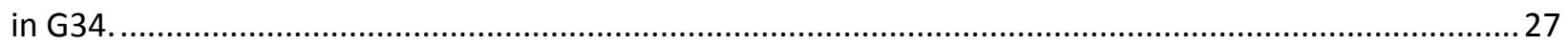

Figure 9| Sensitivity analysis of ABM indicates the importance of cell type subpopulations...................29

Figure 10| Development of an agent-based model to predict cancer cell response to doxorubicin in 3D

tumor bulk to stroma transition environments..................................................................................... 42

Figure 11| Logic flow charts for in silico modelling.......................................................................... 43

Figure 12| Doxorubicin is less cytotoxic to breast cancer cells cultured in fibroblast-conditioned media or

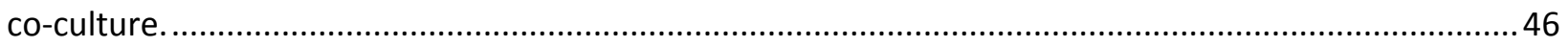

Figure 13 In single co-culture, the ratio of tumor cells to fibroblasts alters the viability of tumor cells in

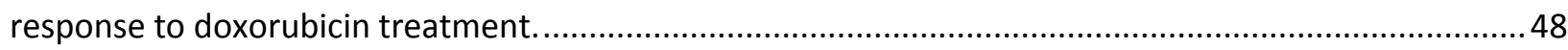

Figure 14| Ratiometric response of tumor cells occurs when cells are co-cultured in a breast-mimetic collagen I matrix............................................................................................................................ 50

Figure 15| Agent-based model (ABM) predicts overall and distance-dependent response of tumor cells

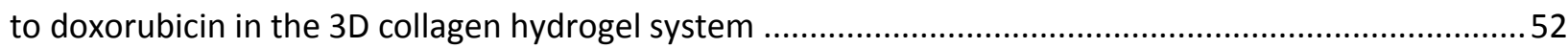

Figure 16| Layered in silico and in vitro models of the tumor to stroma transition zone indicate that tumor cells in the bulk are more viable after doxorubicin than those in the stroma. ..............................54

Figure 17| The agent-based model reveals complex interactions between transport properties and cellular response to chemotherapy across the TST Zone. ..................................................................5

Figure 18| The agent-based model reveals complex interactions between transport properties and concentration profiles............................................................................................................ 58

Figure S1| Fibroblast viability is lessened in the presence of cancer cells within hanging-well 2D culture.

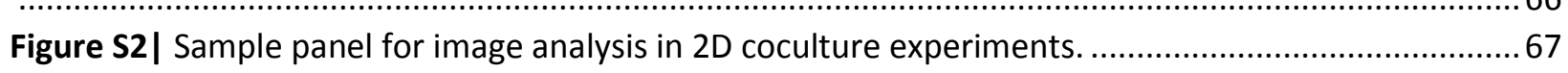

Figure S3| Sample panel for image analysis in 3D coculture experiments. ...........................................68

Figure S4| 2D doxorubicin EC50 curves for HDFs and MDA-MB-231 incorporated into the agent-based models. 


\section{List of Abbreviations}

\begin{tabular}{|l|l|}
\hline ABM & Agent-based model \\
\hline CAF & Cancer-associated fibroblast \\
\hline DOX & Doxorubicin \\
\hline EPR & Enhanced permeability and retention \\
\hline Fb & Fibroblast \\
\hline HDF & Human dermal fibroblast \\
\hline IFP & Interstitial fluid pressure \\
\hline MDA-MB-231 & Human breast adenocarcinoma cell line \\
\hline TC & Tumor Cell \\
\hline TME & Tumor microenvironment \\
\hline TSTM & Tumor to stroma transition model \\
\hline
\end{tabular}




\section{CHAPTER 1: Introduction}

The tumor microenvironment (TME) is composed of varied factors. Cancer cells display unique characteristics that lead to the development of disordered vasculature, hypoxia, elevated $\mathrm{pH}$, and necrosis. Additionally, the developing tumor is affected by several local environment factors including cancer associated cell signaling, extracellular matrix remodeling, and interstitial fluid flow. Together, these TME factors lead to pro-metastatic phenomena.

Therapeutic delivery within the tumor is attenuated by static pressure and the permeability of the tissue, which is mediated by the extent of ECM remodeling. Additionally, cellular signaling from stromal fibroblasts limits the efficacy of treatment. This phenomenon is of particular relevance at the tumor border, where there is a transitional region from predominantly cancer cells to predominantly stromal cells. These factors promote cancer growth and select for a potentially metastatic subpopulation.

Furthermore, interstitial fluid flow at the tumor border affects the migration characteristics of cancer cells. Interstitial fluid flow has been implicated in an increase in cancer cell invasion, which is the major effector of cancer spread and decreased patient viability. In brain cancer, two different mechanisms have been implicated in this increased invasion, and this invasion is mediated by both CXCR4 and CD44.

Therefore, TME complexity necessitates utilization of robust models to elucidate the effects of the TME on cancer development and progression. The current work defines two novel agent-based models that describe and predict cancer specific outcomes within in vitro TME mimetic systems. Our models indicate that brain cancer cell invasion is increased in the presence of interstitial flow, and this increased invasion is driven by two specific mechanisms, CXCR4-CXCL12 autologous chemotaxis and CD44 mechanotransduction. Additionally, in vitro and in silico models of the tumor border transition zone predict that regional viability within the breast tumor border is affected by both fibroblast signaling and transport mechanisms, and this affect could be selecting for important resistant subpopulations. Together, our data describe varied TME factors that mediate cancer cell response, and the utilization of agent-based models has facilitated the prediction, characterization, and explanation of in vitro findings. 


\section{CHAPTER 2: Background}

\subsection{The Tumor Microenvironment}

The tumor microenvironment is generally defined as the local factors that influence the growth and progression of cancer. While the components of the tumor microenvironment are cancer type and context specific, there are several common constituents inherent to tumors. Cancer associated cells (Figure 1F/E), the physical adherent matrix or extracellular matrix (Figure 1D), and biophysical forces (Figure $1 \mathrm{H})$ are several key components of the tumor microenvironment ${ }^{1,2}$, while vasculature is additionally important for drug delivery considerations (Figure 1A). Characterization of the tumor microenvironment provides insight into the mechanisms of cancer migration, proliferation, metastasis, and chemotherapy resistance, thus facilitating better standard of care and increased treatment efficacy.

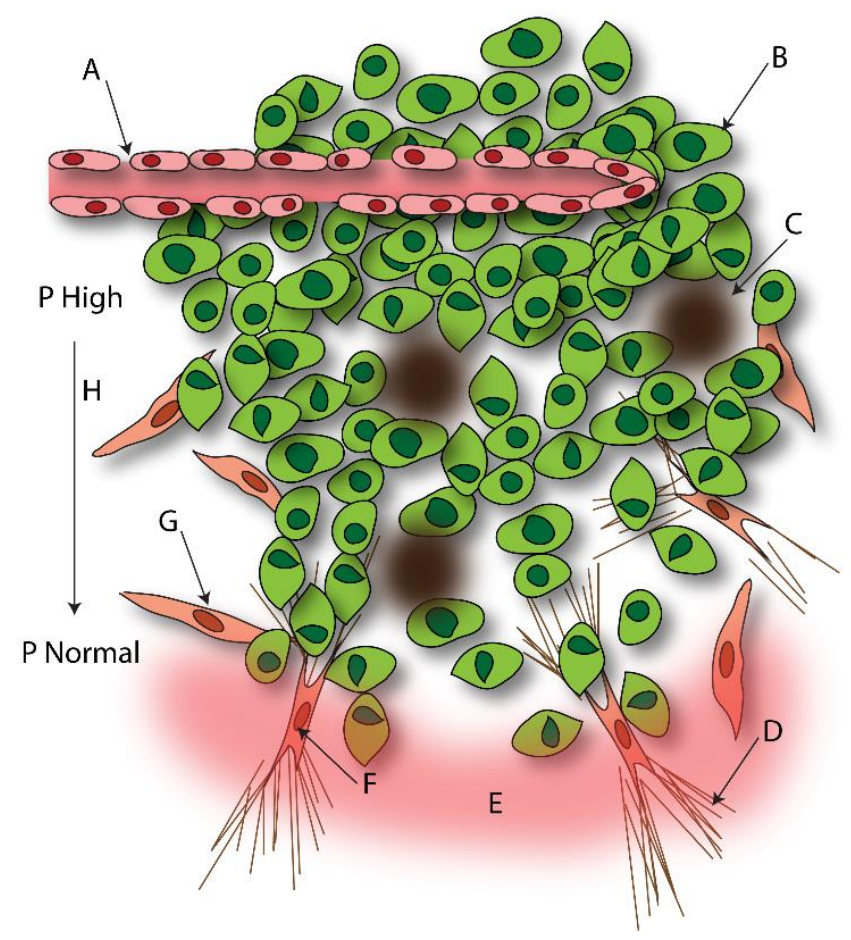

Figure 1|Components of the tumor microenvironment.

The tumor microenvironment is characterized by (A) fenestrated vasculature, (B) cancer cells, (C) necrotic regions, (D) extracellular matrix, (E) chemokine gradients, (F/G) cancer associated cells (activated fibroblasts and normal fibroblasts respectively), and $(\mathbf{H})$ interstitial fluid pressure. 


\subsubsection{Tumor Characteristics}

The most abundant component of the tumor microenvironment is cancer cells (Figure 1B). These cells are defined by several universal hallmarks, classically defined by Hanahan and Weinberg, including growth suppression evasion, mutation, replicative immortality, inflammation, invasion mechanisms, and stimulating angiogenesis ${ }^{3,4}$. These ubiquitous cancer hallmarks facilitate the development of unique tumor characteristics. Unchecked growth coupled with replicative immortality yields increased metabolic activity and associated decrease in $\mathrm{pH}^{5}$ within the tumor microenvironment. Increased metabolic activity additionally causes oxygen depletion ${ }^{6}$ and regions of hypoxia. Therefore, unchecked growth creates a detrimental environment for the cancer cells and subsequent regions of necrosis (Figure 1C). Additionally, this environment creates a selective pressure that yields regional subpopulations that are more likely to proliferate and invade ${ }^{7}$.

The lack of oxygen within tumors causes cancer cells to secrete pro-angiogenic factors to stimulate the growth of new blood vessels ${ }^{8}$ (Figure 1A), however, this tumor vasculature, or neovasculature, is significantly altered from normal tissue blood vessels. Neovasculature is defined by abnormal blood vessel shapes, and large fenestrations caused by misalignment of endothelial cells ${ }^{9}$. These gaps allow cells to intravasate into the vasculature, which has been proposed as a mechanism of metastasis ${ }^{10}$. Interestingly, these neovasculature abnormalities also lead to a distinct phenomenon known as the enhanced permeability retention (EPR) effect. Treatment of tumors with large molecule drugs is enhanced over small molecule treatment due to accumulation into the tumor by extravasation from the leaky vasculature into the tumor bulk, while simultaneously being blocked from extravasation from the vasculature into other organs due to normal epithelial junction size exclusion ${ }^{10}$.

\subsubsection{Tumor Associated Cells}

While cancer cells are the most abundant cell type in the TME, they do not act alone. Propinquity to surrounding stroma yields infiltration of several cell types into the tumor bulk, which then interact with the cancer cells. These cell types include immune cells, such as T lymphocytes and B lymphocytes, 
myeloid cells, such as neutrophils and macrophages, adipocytes, and fibroblasts ${ }^{11}$. T lymphocytes and B lymphocytes either help promote or hinder the growth of cancer depending on the particular secreted factors and the cancer context ${ }^{12,13}$. Tumor associated macrophages are generally considered to be protumorgenic ${ }^{13}$, and these macrophages have been shown to enhance cancer cell migration ${ }^{14}$. Adipocytes recruit cancer cells in specific via adipocyte specific secreted factors ${ }^{15}$.

However, perhaps the most important cell type in the development and progression of cancer is the fibroblast (Figure 1G). Normally, fibroblasts are active in the native wound healing response. After an injury, platelets in the blood activate fibroblasts by releasing transforming growth factor beta (TGF- $\beta$ ); the fibroblasts then facilitate the remodeling and repair of local tissue. Activated fibroblasts secrete proinflammatory and pro-angiogenic cytokines that further stimulate remodeling and repair, and these fibroblasts deposit the structural components of the tissue, or the extracellular matrix $(\mathrm{ECM})^{16}$. After the wound has been healed, the fibroblasts are inactivated or removed from the injury site.

Contrastingly, in chronic wounds such as ulcerative colitis, where fibroblasts remain activated for extended periods of time, the risk of cancer development is much greater than in normal tissue due to the pro-tumorgenic properties of these cells ${ }^{17}$. Interestingly, developing tumors displays many of the characteristics of a chronic wound as inflammation, increased angiogenesis, and matrix remodeling by fibroblasts (Figure 1D) are all present in tumor growth ${ }^{18}$. Cancer associated fibroblasts (CAFs) are the most abundant cell type in the tumor stroma, and cancer cells have been shown to secrete fibroblast activating factors including TGF- $\beta$. Activated fibroblasts in the TME (Figure 1F), in turn, secrete mitogenic growth factors (Figure E), and secrete their own TGF- $\beta$, which suppresses cells in the immune system $^{19}$. Finally, fibroblast-cancer interactions have been directly implicated in decreased chemotherapy efficacy ${ }^{20}$, leading to sustained tumor growth and the potential for subsequent metastasis.

\subsubsection{Extracellular Matrix}

The extracellular matrix $(\mathrm{ECM})$ is defined by the various macromolecules that the give tissue structural integrity and rigidity. These molecules include proteins, glycoproteins, proteoglycans, and 
polysaccharides, with unique physical properties that allow for chemical crosslinking and tissue cellular development ${ }^{21}$. The ECM provides a unique biological scaffold for cell adherence, growth, and development. Cells utilize several signaling mechanisms to respond to biomechanical forces and sense the rigidity of the matrix. The extent of ECM elasticity allows cells to sense external stresses and modulate their behavior, and the elasticity is also driver for cell differentiation and stem cell maintenance ${ }^{22}$. The ECM also provides a structural framework for cellular motility. Signal transduction is modulated indirectly by the ECM because the ECM can act as a signal reservoir. Secreted factors, adhere to the ECM, and cells interact with the bound ligands ${ }^{23}$. The major components of the ECM are variable depending on the tissue of interest, which facilitates varied tissue development and growth. For example, the major component of the breast ECM is collagen 1, while the major component of the brain ECM is hylaronan ${ }^{24}$. Fibroblasts are the main mediators of ECM alteration and remodeling, and they facilitate the deposition and degradation of ECM components; however, this process is precisely controlled by transcriptional and translational regulation ${ }^{25}$.

In tumors, the ECM is significantly altered from normal tissue ECM. Normal regulatory function is disrupted in CAFs, and these fibroblasts actively deposit collagen in the TME, leading to fibrosis and increased stromal density ${ }^{26}$. In addition to upregulation of collagen deposition, matrix degradation enzymes are concurrently downregulated, which leads to further accumulation of ECM in the TME. For example, breast cancer tissue is 10 times stiffer than normal tissue ${ }^{27}$. The orientation of fibers is also altered in tumors. While normal tissue is characterized by random non-oriented fibers, the ECM in tumors is linearized parallel to epithelium, which has been hypothesized to support cellular migration and invasion. In fact, metastatic cancer progression is defined by upregulation of ECM sensing mechanisms such as CD44, and cancer cell populations with these receptors are more likely to survive and spread ${ }^{28}$.

\subsubsection{Biophysical Forces}

Increased ECM deposition, unchecked cellular growth, and compromised vasculature all lead to elevated pressure in the tumor bulk. Interestingly, as classically described by Jain and colleagues ${ }^{29,30}$, this 
pressure is consistently high throughout the tumor bulk, as compared to the tumor border. At the tumor border, the pressure is normal compared to the elevated pressure in the tumor bulk, which drives interstitial fluid from the tumor bulk into the surrounding stroma (Figure 1H). The extent of pressure gradient formation is modulated by the leakiness of the vasculature, and vascular normalization has been shown to decrease IFP $^{29}$. This interstitial flow has implications for disease spread and metastasis as well as therapeutic efficacy. Both of these topics are discussed below.

\subsection{Barriers to Therapeutic Efficacy}

Tumors are notoriously difficult to treat. The combination of leaky vasculature, elevated interstitial fluid pressure, abundant extracellular matrix deposition, adaptive cellular resistance, and CAFtumor cell interactions all lead to attenuated chemotherapeutic efficacy. In general, the obstacles to therapy can be described by physical barriers and cell-mediated barriers. In order to be effective, therapeutic agents must reach the tumor cells; however, several factors mitigate the transport of solute in the TME. Therapy that does reach tumor cells must then overcome barriers of adaptive tumor cell immunity and tumor-associated cell signaling. Together, these factors lead to detrimental patient outcomes and the possibility for disease recurrence.

\subsubsection{Physical Barriers to Therapeutic Efficacy}

As discussed previously, matrix deposition, rapid cellular growth, and disrupted vasculature lead to elevated pressure within tumors. This pressure leads to two distinct physical barriers. Firstly, while large molecule drugs, such as nanoparticles or antibodies, accumulate in the tumor due to the EPR effect, this accumulation is mostly perivascular. Drug penetration into the tumor from the vasculature is limited by a relatively constant pressure throughout the tumor bulk, meaning that diffusion dominates convection in this context. Since large molecules do not readily diffuse, the distribution is localized, and particles are more likely to get trapped in the matrix ${ }^{31,32}$. However, it should be noted that tumor heterogeneity could 
yield a moderate amount of drug penetration depending on the distribution of vasculature and the shape of the tumor ${ }^{33}$.

Secondly, at the tumor border the differential in pressure between the tumor bulk and the surrounding stroma yields fluid efflux from the tumor into the surrounding stroma ${ }^{30,34,35}$. This means that any drug that diffuses to the tumor border, or any drug released by vasculature near the border, will leave the tumor via convective transport. As large molecule delivery is dominated by convection, this is especially problematic for many chemotherapeutics delivered via nanoparticles or targeted antibodies.

However, the ECM composition also affects the extent of interstitial flow independently of the interstitial pressure, as permeability and porosity of the tissue of interest affect the likelihood of fluid transport. Neoplastic tissue ECM displays remarkable heterogeneity ${ }^{36}$, and the resistance to convective flow in the interstitium is dependent on the abundance and type of matrix molecule in the particular TME context. For example, transport in chondrosarcoma, a cartilage tumor, vs hepatoma, a liver tumor, would be significantly different due to greater relative abundance of ECM molecules in cartilage as compared to the liver ${ }^{34}$. Mass transfer of a molecule of molecular weight of $10^{3} \mathrm{~g} / \mathrm{mol}$ would be dominated by diffusion in chondrosarcoma and convection in hepatoma due solely to the abundance of ECM components. Increased ECM secretion by fibroblasts in the TME, therefore, significantly hinders solute transport.

\subsubsection{Cellular Resistance}

In addition to physical barriers, therapeutic efficacy is attenuated by cellular dynamics. Cancer cells can be inherently resistant to drugs due to mutations and adaptations. For example, the above mentioned interstitial fluid flow at the tumor border causes gradients of drugs to form near the tumor edge. These drug gradients provide a selective pressure for adaptive immunity ${ }^{37}$. Several mechanisms have been implicated for multi-drug resistance in cancer cells including upregulation of DNA repair mechanisms, drug metabolism, and drug alteration ${ }^{38}$, however, two of the major effectors of classical drug resistance in cancer is increased drug efflux or decreased drug uptake ${ }^{39}$. ATP binding cassette transporters 
or $\mathrm{ABC}$ transporters have been characterized as major mediators of drug removal from the cell, thus limiting cytotoxic effects. $\mathrm{ABC}$ transporter expression is upregulated in multi-drug resistance, and this mechanism non-specifically yields broad resistance to multiple and varied drugs ${ }^{40}$. Additionally, some cancer cells have observable decrease in drug uptake due to specific downregulation of plasma membrane binding proteins ${ }^{41}$.

While, cancer cells can develop resistance independently, cancer associated cells also play a role in tumor resistance to chemotherapy, and fibroblasts, specifically, have been implicated in cancer cell chemotherapy immunity. Marusyk and colleagues describe cancer cell insensitivity to lapatanib due to coculture and proximity to fibroblasts. This resistance is mediated by decreased drug accumulation and drug interaction with fibroblast secreted factors ${ }^{42}$. Cancer associated fibroblasts also secrete pro-survival factors that limit cancer cell apoptosis and could act to protect cancer cells from chemotherapeutic effects ${ }^{20}$. In the context of neoadjuvant breast cancer therapy, the presence of cancer associated fibroblasts signatures has been directly implicated in poorer survival outcomes ${ }^{43}$, and targeted therapy against these stromal cells has been shown to improve intratumoral uptake of doxorubicin within in vivo murine models ${ }^{44}$.

\subsection{Mechanisms of Invasion and Migration}

Cancer cells are not stationary. These cells utilize several mechanisms to interact with the ECM and migrate. More severe forms of cancer are characterized by the invasive nature of the cancer cells, as cancer progression by metastasis is mediated by cellular invasion and motility. Gliomas, brain tumors, are composed of particularly invasive cell populations, which are highly metastatic, leading to extremely poor patient outcomes. However, the mechanisms of this migration are relatively unknown, necessitating further research and experimentation.

\subsubsection{Cancer Cell Motility}

In general, the motility of cancer is mediated by a distinct phenotypic switch from epithelial cell types to mesenchymal cell types. This switch is defined by lack of E-cadherin expression. Cadherins are 
cell-cell adhesion proteins that allow cells to adhere to surrounding cells, and disruption of this particular cadherin leads to disruption of tight junctions and cell polarity, which frees the cancer cell from close contact with surrounding cells ${ }^{45}$. Disruption of E-cadherin function has also been implicated with the loss of cell-matrix adhesion mechanisms, thus freeing the cancer cells and promoting unhampered migration and associated metastasis ${ }^{46}$.

In addition to a decrease in adhesion, migratory cancer cells also display an alteration of gene expression related to cancer cell invasion. CXCR4, a receptor associated with directed cellular motility toward the complementary ligand CXCL12, is over-expressed in many cancers ${ }^{47}$. Vimentin, a filament protein associated with mesenchymal motility ${ }^{48}$, cathepsin and $\mathrm{MMPs}^{49}$, proteases that degrade matrix, integrins ${ }^{50}$, ECM adhesion molecules associated with signaling, $\mathrm{CD} 44^{51}$, a matrix adhesion protein and signal transducer, and $\mathrm{RhoA} / \mathrm{C}^{52}$, proteins that generate contractile force, are all overexpressed in invasive cancers. The combination of extracellular matrix degradation, increased motility, contractile force generation, directed motility, and heightened signal transduction could, therefore, all mediated cancer motility and metastasis.

\subsubsection{Interstitial Flow and Increased Invasion}

While many mechanisms have been implicated with migration in general, research indicates that interstitial flow enhances the invasion of cancer cells $\mathrm{s}^{53,54,55}$ as compared to normal motility levels. Since interstitial flow is dominant at the tumor border, this increased invasion could be a major factor mediating cancer metastasis $^{53}$. Two mechanisms have been proposed for this increased invasion with interstitial flow. Firstly, it is known that the migratory receptor CXCR4 expression is upregulated in invasive cancer cells $s^{55,47}$, however, certain cell populations have both the receptor (CXCR4) and secrete the ligand for that receptor (CXCL12). In flow conditions, it is hypothesized that secreted CXCL12 is convectively driven away from the cell secreting the ligand, thus forming a gradient of CXCL12 in front of the cell in the flow direction (Figure 2A) ${ }^{54,55}$. If a cell has the receptor for CXCR4, it will migrate in the direction of flow in response to the stimulatory gradient. This directed invasion is termed autologous chemotaxis ${ }^{54}$. 
It has also been shown that CD44 expression is upregulated in migratory cancer cells ${ }^{51}$. CD44 mediates migration via signal transduction due to interaction with the ECM, and it has been shown that blocking of this receptor can alleviated increased invasion in the presence of interstitial flow ${ }^{56}$. Rousseau further describes the importance of CD44 as a mediator of dimerization and activation of other receptors, notably CXCR4, through interaction with the ECM and mechanotransduction ${ }^{57}$. It is therefore possible that increased invasion in the presence of interstitial flow is also mediated by a mechanotransduction mechanism and concurrent phosphorylation of CXCR4 (Figure 2B).

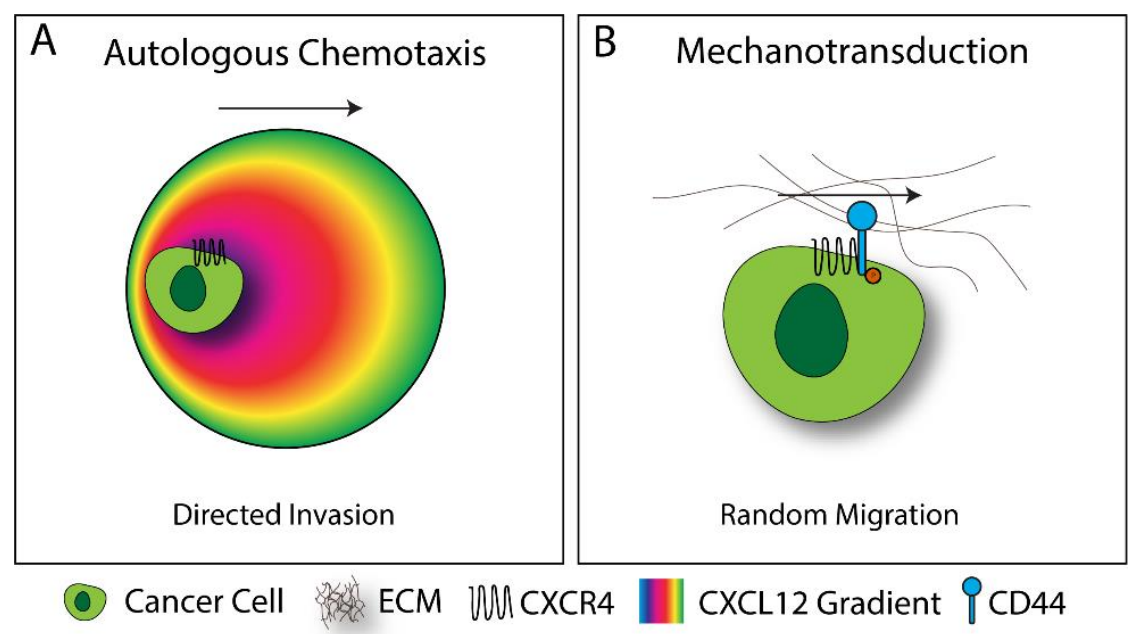

Figure 2| Proposed dominant mechanisms for increased invasion with interstitial flow. (A) Directed invasion of cells via CXCL12 gradient development under flow conditions and response by CXCR4. (B) $\mathrm{CD}_{4} 4^{+} / \mathrm{CXCR} 4^{+}$dimerization and signal transduction for random migration under flow conditions.

\subsection{D In Vitro Models of the Tumor Microenvironment}

As the tumor microenvironment is very complex, and there are many factors that influence the progression and spread of cancer, it is necessary to develop relevant experimental models to study cancer in an appropriate environment. 2D culture models are the easiest method to study cancer, however, research has shown that $2 \mathrm{D}$ culture models are not always relevant for the unique $3 \mathrm{D}$ environment observed in vivo. ${ }^{58}$ Murine models are the current standard for experimental and preclinical cancer research, however, these models are complex and are less controllable than in vitro models. Therefore, 
bridging this experimental gap with simple and relevant 3D culture models provide medium throughput results comparable to in vivo conditions. Several in vitro systems have been developed to study cancer within the 3D context.

\subsubsection{Simple 3D Models}

Perhaps the simplest 3D models utilized to study cancer are tumor spheroids. Spheroids are 3D masses or cellular clusters within a cell culture dish that are grown from single cell suspensions. These masses replicate many of the properties inherent to in vivo tumors including cell to cell interactions, regions of hypoxia, elevated $\mathrm{pH}$, and natural ECM deposition ${ }^{42}$. Spheroids can also be utilized for coculture studies as other cell types can be incorporated into the tumor spheroids ${ }^{59}$. Spheroids are especially optimal for drug screening studies, as the 3D spheroids display regional heterogeneity replicative of the actual tumor ${ }^{60}$.

Despite several advantages, tumor spheroids are also limited. Firstly, several types of cancer do not form spheroids ${ }^{60}$; therefore, these models cannot be utilized. Secondly, while ECM components can be incorporated into spheroids, the abundance and type of components are not always replicative of the real tumor environment ${ }^{61}$. Additionally, the morphology and structure of the spheroids is highly variable. While this is a benefit in terms of developing varied tumor-like structures, it is not advantageous if the composition of the tumor is to be tuned experimentally ${ }^{60}$. Finally, the method of spheroid formation does not account for interstitial flow dynamics.

\subsubsection{ECM Hydrogel Models}

In order to address the limitations of simple 3D models, hydrogel scaffolds have been developed to replicate the 3D tumor microenvironment. Natural hydrogel scaffolds are characterized by the utilization of physiologically relevant ECM components to create a structurally stable 3D growth medium for cancer cell development and study ${ }^{62}$. These models are robust, as the ECM composition can be tuned to relevant concentrations and the dominant types of ECM components can incorporated to create 
hydrogels with mechanical properties similar to the tissue of interest ${ }^{63}$. Additionally, cancer associated cell types can be co-cultured in these hydrogels for cell to cell interaction characterization, and the ratios of cells can be tuned to values observed in vivo ${ }^{64}$. Finally, incorporation of fluid on top of these hydrogels introduces a flow rate through the gel comparable to the interstitial flow rate observed within tumors, thus providing an experimental model for the study of interstitial flow effects on cancer cells $\mathrm{s}^{54,55,64}$.

\subsection{Agent-Based Models}

The same complexities that necessitate robust in vitro models also demand appropriate in silico modeling platforms. In silico research methodologies provide a supplementary data analysis context that can enhance and predict biological phenomena. Agent-based models (ABMs) are particularly suited for biological applications, as they can predict and describe complex spatial and temporal biological interactions ${ }^{65,66}$. In particular, the benefit of agent-based models is the emergent behavior that is possible through incorporation of specific model components. In essence, the sum of many components yields results that would not be predicted by the individual components separately.

\subsubsection{An Introduction to Agent-Based Models}

Agent-based models are composed of several key components. The most important components of these models are the agents, and there are two types of agents utilized in these models, patches and turtles. Patches are equivalently sized squares that make up the coordinate grid of the agent-based model. The user defines a spatial framework for the model that has dimensions $\mathrm{N} \times \mathrm{N}$ where $\mathrm{N}$ is the number of patches in either a row or a column. For example, a spatial framework of 4 x 4 would have a total of 16 patches in a symmetric square grid. Each patch is unique, and can be utilized within the model. For example, a patch can be programmed to store a specific variable and the user can reference that variable later.

The second component of these models are turtles. Turtles are the objects that interact on top of the patch grid. Turtles are dynamic objects that can move, grow, shrink, die, etc based on the desired 
outcome and the programmed rules of the model. Turtles can also store variables, like the patches, and the turtles can also receive variables from patches beneath them or transfer them to other turtles. The user can specify as many types of turtles as desired, and each individual turtle or each type of turtle can be programmed with different governing behavior.

The behavior of the agents is updated at successive intervals determined by the user and outputted to a display screen. Much like individual frames in a movie or animation, the location, behavior, size, etc of the turtles and/or patches is updated dynamically according to the specific programming methods. This yields a viewable dynamic model with temporal aspects controlled by the degree of change between frames. For example, if the user desired to create an agent-based model of a car moving rightward at a speed of $20 \mathrm{mph}$, the user could specify a patch grid of $20 \times 20$ with each patch being 5 miles long. At each individual frame, the user specified program could tell the car turtle to move rightward by a one patch distance on the coordinate grid. If the time between two frames was specified as 15 minutes, iterating this method for four frames would move the car rightward by 20 miles in 60 minutes or $20 \mathrm{mph}$. This method could then be iterated as necessary.

\subsubsection{Agent-Based Models of Cellular Motility}

As agent-based models are inherently spatial, agent-based models characterizing cellular motility have been previously utilized. Emmonet et al. describe an agent-based model of bacterial chemotaxis with and without chemo-attractant using the predictive equations developed from classical microbiology. Here, motility in the absence of attractant is defined by random motility synonymous to chemical diffusion ${ }^{67}$. Enderling and colleagues developed an agent-based model of tumor development that incorporates migration programming methods in order to predict tumor morphology ${ }^{68}$. Interestingly, Heiko and Enderling also explore tumor development as a result of random migration and chemoattractant-mediated migration. Here the morphology is dependent on the directionality of migration ${ }^{69}$. 


\subsubsection{Agent-Based Models in Pharmacology}

While agent-based models are not utilized as readily for the dynamics of chemotherapy, as other modeling methodologies are more ubiquitous, model-based descriptive techniques are being utilized in the area of pharmacology. These models help to facilitate hypothesis testing and characterize complex dynamics in a simple and replicable platform ${ }^{70}$. For example, an agent-based model has been used to predict the effect of novel drug targets on the fungal infections based on either inhibition of fungal growth or phenotypic transition ${ }^{71}$. Agent-based models have also been utilized concurrently with descriptive pharmacokinetics and pharmacodynamics to predict the effect of drugs at the cellular level ${ }^{72}$. In general, however, it appears that many models are context and hypothesis specific, and the utilization of agentbased models to describe treatment dynamics is an emerging field ${ }^{70}$.

\subsection{Motivation and Conclusion}

The tumor microenvironment is very diverse; the combination of cellular factors, ECM, biophysical forces, and therapeutic barriers all contribute to complexity, which is difficult to characterize and difficult to study. Therefore, robust research methods must be employed to elucidate the effects of this unique environment on the development and spread of cancer. In vitro hydrogel systems utilized concurrently with robust in silico agent-based models facilitate the discovery of TME specific tumor outcomes in the context of interstitial flow and chemotherapy treatment. Here we describe two agentbased models that predict the effects of interstitial flow on brain cancer invasion and the effects of fibroblasts co-culture on cancer cell viability in the presences of chemotherapy.

While agent-based models have been utilized to describe cellular motility ${ }^{67,68,69}$, these models have not incorporated cellular invasion as a specific outcome measure. Additionally, interstitial flow was not a factor in any of the above models. Here we describe a novel agent-based model that replicates our in vitro invasion assay and predicts mechanisms of interstitial flow-mediated increased invasion of glioma cells. 
Agent-based models describing the effects of therapy are limited, however, it is clear that these models provide insight into complex systems, and can be utilized in this context ${ }^{70}$. Here we describe a novel agent-based model that predicts the region specific response of cancer cells to chemotherapy within the tumor border to stroma transition region based on the kinetics of therapeutic transport and the protective effect of fibroblasts. We have concurrently developed a layered hydrogel system of the tumor border to stroma transition zone. Regional variability in this in vitro model, in response to chemotherapy, further elucidate the importance of the TME in mediating cancer cell resistance to chemotherapy. 


\section{CHAPTER 3: An Agent-Based Model of Cancer Cell Invasion and Migration}

\subsection{Introduction}

Cancer cell motility is linked to decreased patient survival due to metastasis and evasion of treatment. Glioblastoma, brain cancer, is particularly deadly as the cancer cells are characteristically invasive $^{73,74}$. This effect is enhanced by interstitial flow at the tumor border. Interstitial flow develops due to a pressure differential between the tumor bulk and the surrounding tissue, which leads to fold increases in flow velocities ${ }^{75}$. This interstitial flow has been shown to increase the invasion of the cancer cells ${ }^{55}$. Since the standard of care involves surgical resection of the primary bulk, which often misses cells at the invasive edge of the tumor, this population is optimally primed for further invasion ${ }^{76}$.

Increased invasion due to interstitial flow has been predicted by two distinct mechanisms. Firstly, autologous chemotaxis, mediated by cell populations expressing both the migratory receptor CXCR4 and secreting the ligand to that receptor CXCL12, has been proposed by Munson et al. ${ }^{55}$ as a dominating mechanism for increased invasion. These cells secrete CXCL12 which is convectively drive by interstitial flow. This mediates the creation of autologous gradients of CXCL12, which the cell can respond to with its CXCR4 receptor.

Tarbell and colleagues have also described an alternative mechanism in several cancer cell lines $^{56}$. This mechanism involves CD44 mechanotransduction, where cancer cells respond to interstitial flow indirectly by mechanical sensing by the CD44 receptor. It has further been shown that CD44 can phosphorylate other membrane proteins, including CXCR4, which indicates that the increase in flowmediated invasion could also be mediated by CXCR4 phosphorylation by CD44. Here, both in vitro and in silico experimental models have been utilized to describe flow-mediated invasion, and they have been utilized to predict the dominating mechanism of this motility. Additionally, specific subpopulations have been implicated in mediation both mechanotransduction and autologous chemotaxis. 


\subsection{Materials and Methods}

\subsubsection{Glioma Stem Cell Culture}

G34 cells were primary derived as described and were provided as a kind gift from Dr Jakub Godlewski (Cleveland Clinic) and have been previously published and characterized. ${ }^{77}$ Cells were cultured following the previously described protocol. ${ }^{77}$ Briefly, GSCs were cultured in Neurobasal media (ThermoFisher) with N2 and B27 without vitamin A supplements (ThermoFisher), human recombinant bFGF and EGF (50 ng/mL, ThermoFisher), Glutamax (ThermoFisher) and Penicillin-Streptomycin (ThermoFisher) in low-adhesion tissue culture flasks (Grenier). [Methods modified from Kingsmore et al.]

\subsubsection{Three-Dimensonal (3D) In Vitro Interstitial Flow Model}

Hyaluronan is the most abundant extracellular matrix element in the brain and was therefore chosen to be a primary matrix component of our 3D in vitro model. ${ }^{24}$ This matrix gel replicates both the stiffness and composition of the brain, but also allows us to achieve comparable flows that have been reported in vivo. ${ }^{55,78}$ Glioblastoma stem cells were resuspended in hyaluronan-collagen matrix at a density of 1 million cells $/ \mathrm{ml}$ as previously described.$^{55}$ Briefly, cells were resuspended in $50 \mu 1$ of $0.2 \%$ hyaluronan (ESIBIO) /0.12\% rat tail collagen I (Corning) and cross-linked with PEGDA (ESIBIO). This solution was applied to an 8-micron pore 96-well tissue culture insert system (Corning), and allowed to gel for 2 hours. ${ }^{55}$ Interstitial flow was applied by creating a pressure head atop the gel of approximately $1 \mathrm{~cm}$ with GSC media to yield an average superficial velocity of $0.15-2 \mu \mathrm{m} / \mathrm{s}$ (Figure 3B). For static conditions (Figure 3A), the pressure head was removed. After 18 hours in a $37^{\circ} \mathrm{C} / 5 \% \mathrm{CO}_{2}$ incubator, gel containing nonmigrated cells was removed and inserts fixed in $2 \%$ paraformaldehyde. Tissue culture insert bottoms were stained with DAPI (Sigma, St. Louis, MO) and counted in five representative images. \% Invasion was calculated as previously described..$^{55,79}$ 
A

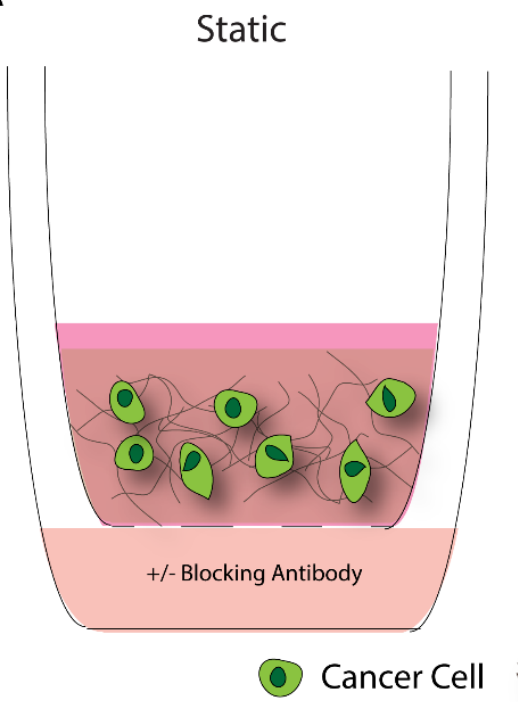

B

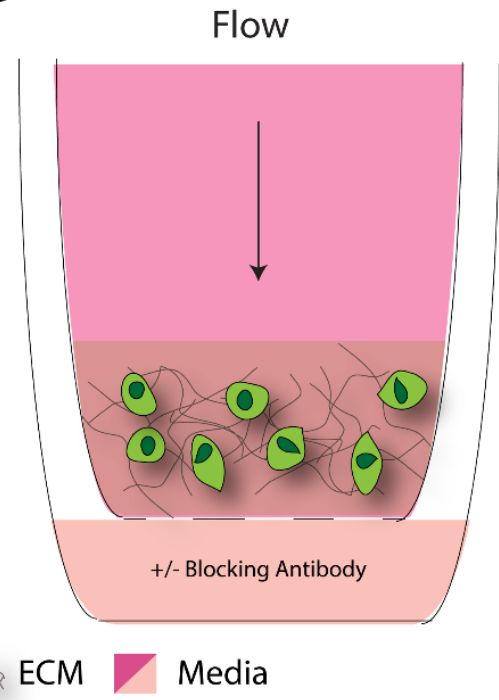

Figure 3| Experimental setup of in vitro hydrogels for invasion studies

(A) Static control for baseline motility without flow. (B) Hydrogel system with pressure induced flow

For blocking antibody studies, anti-CXCR4 (R\&D, clone 44716, $5 \mu \mathrm{g} / \mathrm{mL})$, anti-CXCL12 (R\&D, clone 79014, $50 \mu \mathrm{g} / \mathrm{mL}$ ), anti-CD44 (ThermoFisher, clone 5F12, $10 \mu \mathrm{g} / \mathrm{mL}$ ), or Isotype Control (R\&D, clone $11711,50 \mu \mathrm{g} / \mathrm{mL}$ ) antibodies were added to gel solution and media. All experiments were run independently at least three times (as defined in figure captions), and each experiment had three technical replicates per experimental condition averaged to yield the value for a single $\mathrm{n}$ used in statistical analyses. [Methods modified from Kingsmore et al.]

\subsubsection{Flow cytometry}

Cells in suspension cultures were incubated for 14 hours with Brefeldin A ( $5 \mathrm{ug} / \mathrm{ml}$, Sigma). To identify cell populations, flow cytometric analysis was performed with Fixable Live/Dead Stain (Life Technologies), APC- mouse anti-human CXCR4 (12G5, eBioscience), PE-rat anti-human CD44 (IM7, eBioscience), and PerCP- mouse anti-human CXCL12 (79018, R\&D) along with appropriate isotype controls. Flow cytometry was run using a Millipore Guava 8HT system with data analyzed and graphs 
generated using the accompanying proprietary software (EasyCyte). [Methods modified from Kingsmore et al.]

\subsubsection{Agent-Based Cell Migration Model Construction}

The agent-based model (ABM) was constructed using the Repast Java framework and the Relogo Java package. Cell types were determined by the permutation of all receptor and/or ligand conditions (Table $1)$.

Table 1| Definition of all cell types used in the motility agent-based model

\begin{tabular}{cccc} 
Cell Type & CXCR4 $^{+}$ & CD44 $^{+}$ & CXCL12 $^{+}$ \\
\hline A & + & + & + \\
B & + & + & - \\
C & + & - & - \\
D & - & + & + \\
E & - & - & + \\
F & - & - & - \\
G & + & - & + \\
H & - & + & - \\
\hline
\end{tabular}

Migration behavior was defined by the receptors and/or chemokines associated with each defined cell type, the presence or absence of interstitial flow, and the blocking conditions (Figure 4). Of note, all CXCR $4^{+} \mathrm{CXL} 12^{+}$double positive cells (Type A/G) invade semi-randomly toward the bottom of the gel in the presence of interstitial flow, while all $\mathrm{CD} 44^{+} \mathrm{CXCR} 4^{+}$double positive cells (Type $\left.\mathrm{A} / \mathrm{B}\right)$ migrate randomly in the presence of interstitial flow. By contrast, all CXCR $4^{+} \mathrm{CXL} 12^{+}$double positive cells migrate randomly under static conditions due to autocrine CXCL12 signaling; while, $\mathrm{CD} 44^{+} \mathrm{CXCR} 4^{+}$double positive cells, similar to other cell types (Type $\mathrm{C} / \mathrm{D} / \mathrm{E} / \mathrm{F} / \mathrm{H}$ ), only display baseline motility. Baseline movement was established as $10 \%$ random motility of all cell types regardless of the model conditions, to account for inherent cellular mechanisms of migration other than our receptor-ligand pairs, and it was assumed that CD44 does not activate CXCR4 in the absence of flow. Therefore, cell types $A / B / G$ have 
increased motility under flow as compared to static conditions, with all cells, of these types, moving either randomly or semi-randomly.

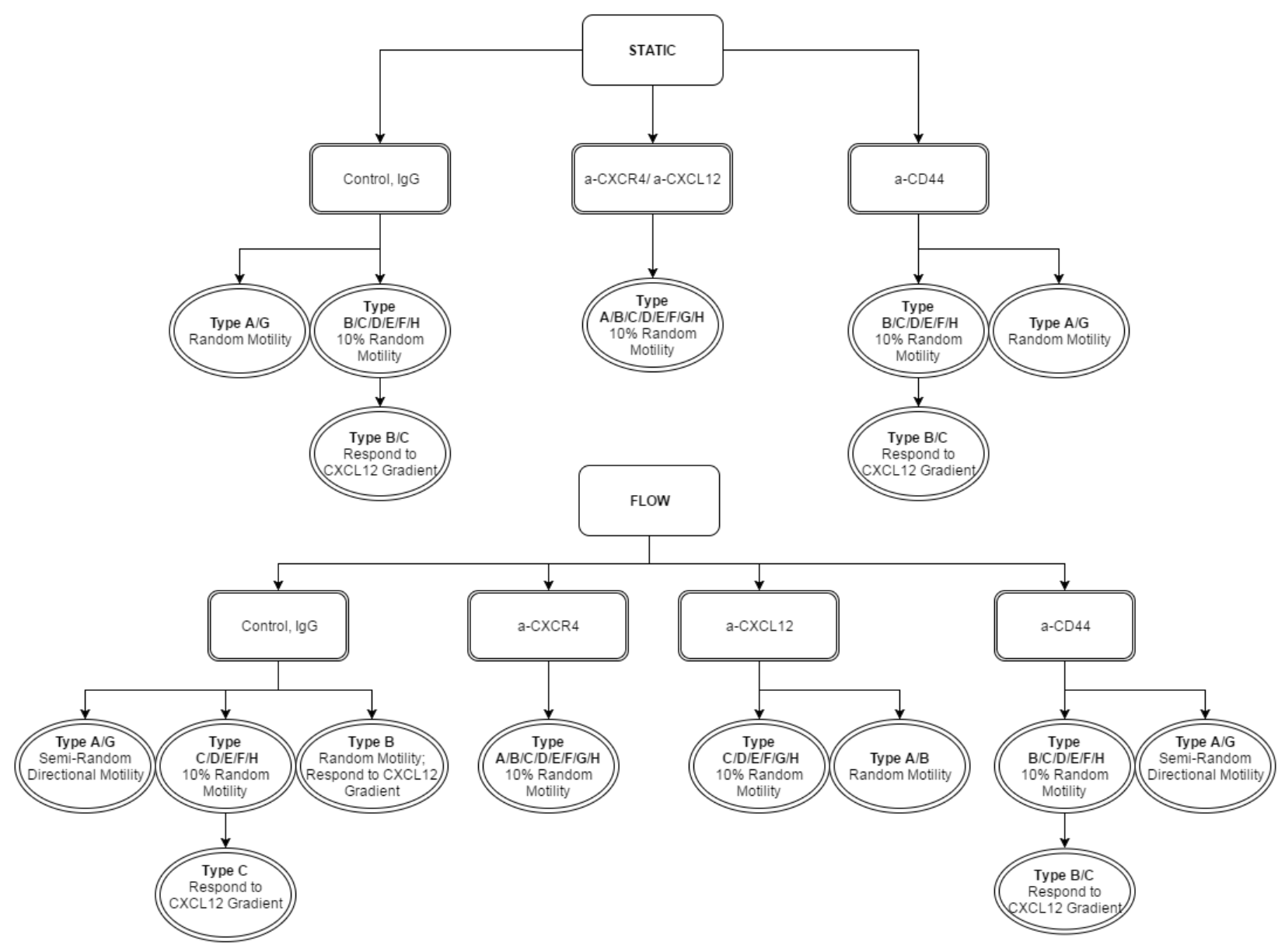

Figure 4| Agent-based model logic flow chart.

Model simulations include static and flow conditions coupled with simulated blocking of relevant receptors or chemokines.

Semi-directional invasion was implemented by introducing one movement in the flow direction for every 13 random movements (Figure 5). Each tic of the scheduling method was defined as one minute, and invasion percentages were produced after an 18 hour timespan. The velocity of the cells was defined as 7 $\mu \mathrm{m} / \mathrm{min}$ as calculated by Munson et al. previously, and the $2 \mathrm{D}$ coordinate grid was initiated to model a vertical cross-section of a tissue culture insert with dimensions of $4.5 \mathrm{~mm}$ x $3.5 \mathrm{~mm}$ ( 7 by 9 patch box grid with side length equal to $0.5 \mathrm{~mm}) .{ }^{55}$ The number of total cells (2800) was established by the number of cells 
in a finite slice of an experimental gel. The percentage of each type of receptor positive/negative cell was determined from flow cytometry data, and the baseline brain cancer model was created from the range of the percentages for all glioma stem cells.

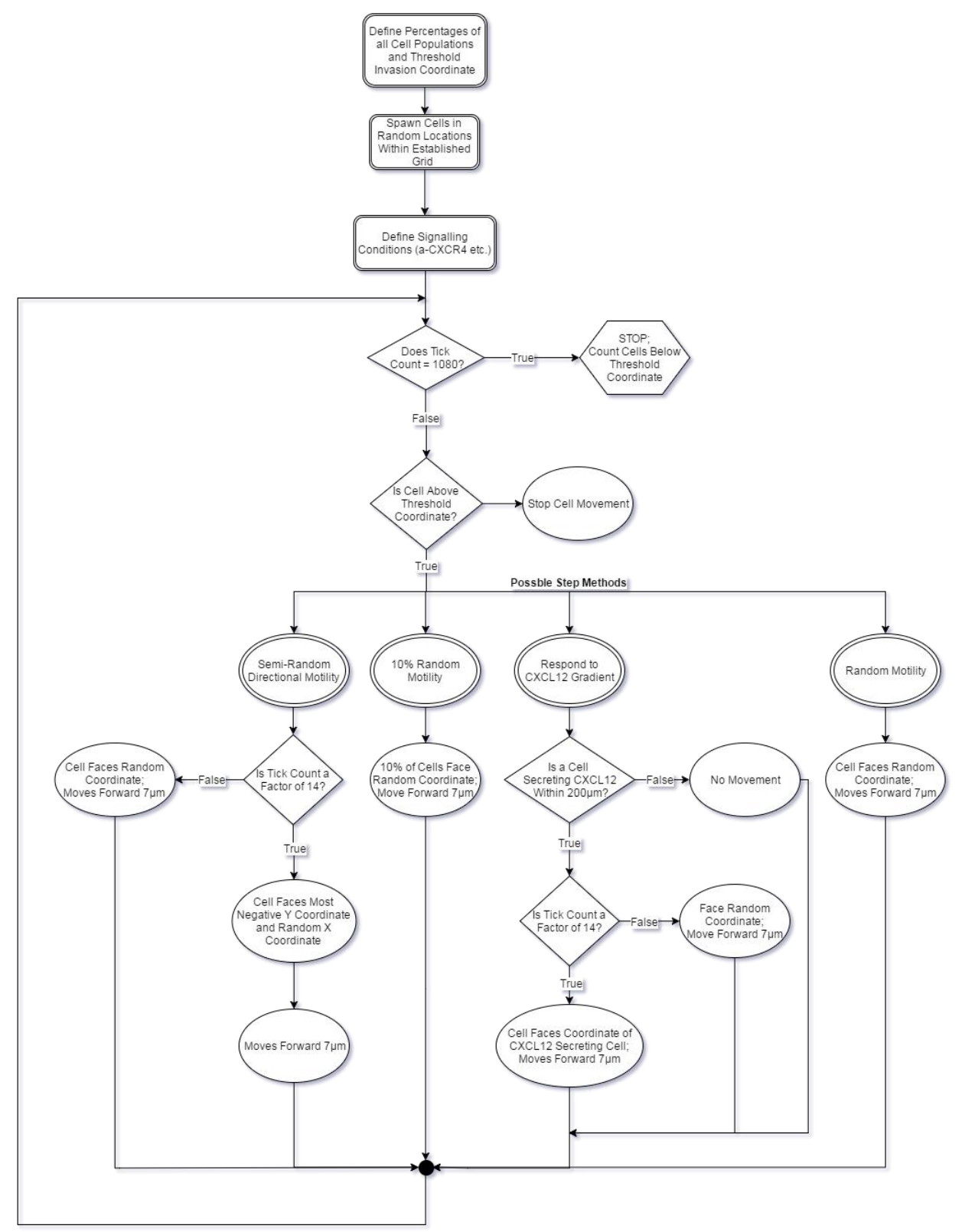

Figure 5| Specific ABM methods flow chart.

Programming methods for each type of motility utilized in the agent-based model. 


\subsubsection{Random Motility Validation}

As random motility was an essential component of the computational model, the random motility programming method, described by facing cells in random directions at each scheduling tic and moving them forward a distance related to their average velocity, was validated using protocols established to describe bacterial motility in the absence of attractant.

In 1967 Adler and Dahl pioneered a method for determining bacterial motility by relating displacement to a motility coefficient synonymous to a diffusion coefficient. ${ }^{80}$ The authors described a general equation for the diffusion of glucose in a gel based on experimentally observed behaviors

$$
\ln \left(\frac{c}{c^{0}}\right)=-\frac{1}{2} * \ln (\pi D t)-\left(\frac{x^{2}}{4 D t}\right)
$$

where $c^{0}$ is the initial concentration, $\mathrm{c}$ is the concentration at distance $\mathrm{x}$ measured from the origin at time $\mathrm{t}$, and $\mathrm{D}$ is the diffusion coefficient of the substance. The authors noted that, in the case of their experimental setup, the first term of the equation was negligible, and the equation could be written as

$$
x^{2}=-4 D t * \ln \left(\frac{c}{c^{0}}\right)
$$

where the variables are described above. The authors additionally noted that the migration of bacteria within gels matched the trends observed with glucose diffusion. This lead to an equation of squared displacement of bacteria

$$
x^{2}=-4 M t * \ln \left(\frac{c}{c^{0}}\right)
$$

where $\mathrm{M}$ is motility coefficient synonymous to a diffusion coefficient, $\mathrm{c}$ is the minimum number of detectable bacteria, and $c^{0}$ is the number of bacteria added to the assay.

Emonet et al. provide a variant of the equation established by Adler and Dahl (Equation 1) in their agent-based AgentCell model of bacterial motility ${ }^{67}$

$$
C(r, t)=\frac{N_{0}}{(4 \pi D t)^{\frac{3}{2}}} * \exp \left(-\frac{r^{2}}{4 D t}\right)
$$


where $\mathrm{C}$ is the concentration of cells at radial distance $\mathrm{r}$ from the origin at time $\mathrm{t}, N_{0}$ is the number of cells present in the model, and $\mathrm{D}$ is the random motility coefficient. This equation can be rearranged into the form

$$
\ln \left(\frac{C(r, t)}{N_{0}}\right)=-r^{2} * 4 D t-\frac{3}{2} \ln (4 \pi D t)
$$

which is almost identical to the equation determined by Adler and Dahl except for an additional term $(3 / 2)$ to account for the $3^{\text {rd }}$ dimensionality of the agent-based model. This equation simplifies to

$$
r^{2}=-4 D t * \ln \left(\frac{C(r, t)}{N_{0}}\right)
$$

assuming that the second term in Equation (5) is negligible, as stated by Adler and Dahl. This expression is equivalent to

$$
<r^{2}>=2 d D t
$$

where $\left\langle\mathrm{r}^{2}\right\rangle$ is ensemble mean squared displacement of all cells and $\mathrm{d}$ is the dimensionality of the system. This is clear from the explanation and analysis of the agent-based model by Emonet et al. This implies that a plot of the ensemble mean squared displacement of all the cells from the origin over time would yield the random motility coefficient $\mathrm{D}$, where $\left\langle\mathrm{r}^{2}\right\rangle$ is additionally defined as ${ }^{81}$

$$
<r^{2}>=\frac{1}{k} \sum_{i=1}^{k} m d_{i}^{2}(\Delta t) ; m d^{2}=\frac{1}{n} \sum_{i=1}^{n} r_{i}^{2}(\Delta t)
$$

where $m d_{i}^{2}(\Delta t)$ is the mean squared displacement at a particular time step $r_{i}^{2}(\Delta t)$ is the squared displacement of a cell from the origin at a particular time step. It should be noted that the squared displacement $r_{i}^{2}(\Delta t)$ is calculated as follows

$$
r_{i}^{2}(\Delta t)=\left(x_{t}-x_{0}\right)^{2}+\left(y_{t}-y_{0}\right)^{2}
$$

in order to normalize displacement of the cells at the zero coordinate in an $\mathrm{x}-\mathrm{y}$ grid, where $\mathrm{x}_{\mathrm{t}}$ is the $\mathrm{x}$ coordinate at time $\mathrm{t}, \mathrm{y}_{\mathrm{t}}$ is the $\mathrm{y}$ coordinate, $\mathrm{x}_{0}$ is the $\mathrm{x}$ coordinate at time zero, and $\mathrm{y}_{0}$ is the $\mathrm{y}$ coordinate at time zero.

An agent-based model of random E.coli cell motility was developed and compared to the results determined by Emonet et al. Cells were spawned in the center of a 32 by $32 \mathrm{~mm}$ coordinate system. For 
every step of the agent method, all cells faced toward a random coordinate within 360 degrees of the cell. All cells then moved forward at a constant velocity toward each associated random coordinate. This procedures was repeated for the desired timeframe. The mean squared displacement relative to time was then calculated (Equations 8-9) using the stored coordinate readings for all the cells at each step of the agent model. The motility coefficient was then calculated (Equation 7) and compared to the model output described by Emonet et al. The velocity of the cells was adjusted based on the relative error of the diffusion coefficient compared to the value stated by Emonet et al.

After confirming the validity of the agent model approach for modelling E.coli motility, the iterative method described above was applied to determine an agent-based model for the random motility of cancer cells in the absence of fluid flow and attractants, using the velocity of cancer cells determined by live imaging. The simulation included 1000 cells and a coordinate system of 100 by 100 um with each model step corresponding to one minute for 250 minute simulation.

\subsubsection{Statistical Analysis}

Statistical analyses were run using Graphpad Prism. Paired t-tests and two-way ANOVA were used for analysis of same subject groups. Unpaired t-tests and two-way ANOVA were used for analysis of independent experimental groups and computational data. . Multivariate ANOVA was used to compare generated curves for sensitivity analyses of the agent-based model. For all data, $* \mathrm{p}<0.05, * * \mathrm{p}<0.01$, and $* * * \mathrm{p}<0.001$, and graphs are given as mean $+/$ - standard error of the mean.

\subsection{Results}

\subsubsection{Simulated random motility is representative of experimental cellular motility in the absence of} stimulant

Comparison of the simple random bacterial ABM to the AgentCell model developed by Emonet et al. showed that at a simulated velocity of $0.058 \mathrm{~mm} / \mathrm{s}$, the random motility coefficient was determined to be $3.99 * 10^{-6} \mathrm{~cm}^{\wedge} 2 / \mathrm{s}$ (Figure $6 \mathrm{~A}$ ). This was compared to the value of $4.21 * 10^{-6} \mathrm{~cm}^{\wedge} 2 / \mathrm{s}$ reported by 
Emonet et al. It should be noted that 400 cells were used instead of the 1166 stated by Emonet et. al. due to size limitations of output excel files, and the difference in slope is accounted for by the dimensionality difference (2D vs 3D). The histogram of displacement $r$ was comparable to the normal trend observed in the AgentCell model (Figure 6B). The trend followed a normal distribution, with mean of $1.39 \mathrm{~mm}$, as confirmed by a normal probability plot.
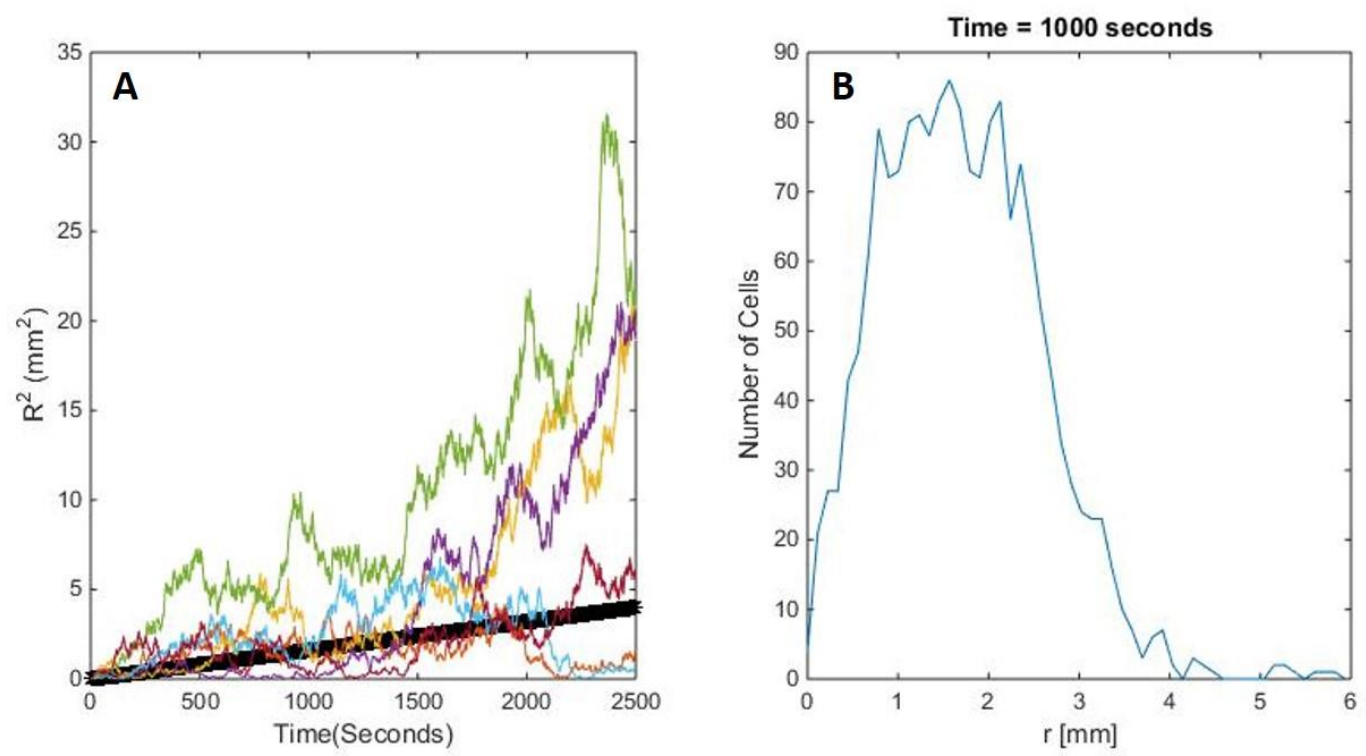

Figure 6| Replication of supplemental Figure 5 from Emonet et al. for validation of agent model. (A) plot of representative squared displacement of four cells from the origin relative to time (here six representative cells are displayed). The best fit line indicates average mean squared displacement for 400 cells with a slope related to the random motility coefficient $\mathrm{D}=3.99 * 10-6 \mathrm{~cm}^{\wedge} 2 / \mathrm{s}$. (B) A plot of number of cells at particular displacement $\mathrm{r}$ at 1000 seconds (1000 cells total, bin size $0.112 \mathrm{~mm}$ ).

After validation of the random motility with bacterial cells, the same method was used to determine the motility coefficient for glioma cells in the absence of fluid flow for both simulated displacements (Figure 7A) and experimental cell displacement (Figure 7C). The agent model motility coefficient obtained from calculating the mean squared displacement for all cells (Equations 7-9) at a cell velocity of $1.077 \mathrm{um} / \mathrm{s}$ was determined to be $2.39 * 10^{-9} \mathrm{~cm}^{2} / \mathrm{s}$. This was compared to the experimentally determined motility coefficient of $2.38 * 10^{-9} \mathrm{~cm}^{2} / \mathrm{s}$. The percentage of cells above 1.2 um in the positive y direction was also determined for the agent model (Figure 7B) and experimental conditions (Figure 7D). The trend was similar with a maximum between 0.35 and 0.45 for both cases. Any discrepancy was most 
likely due to the limited number cells in the experimental conditions (60 cells) compared to the agent model (1000 cells).
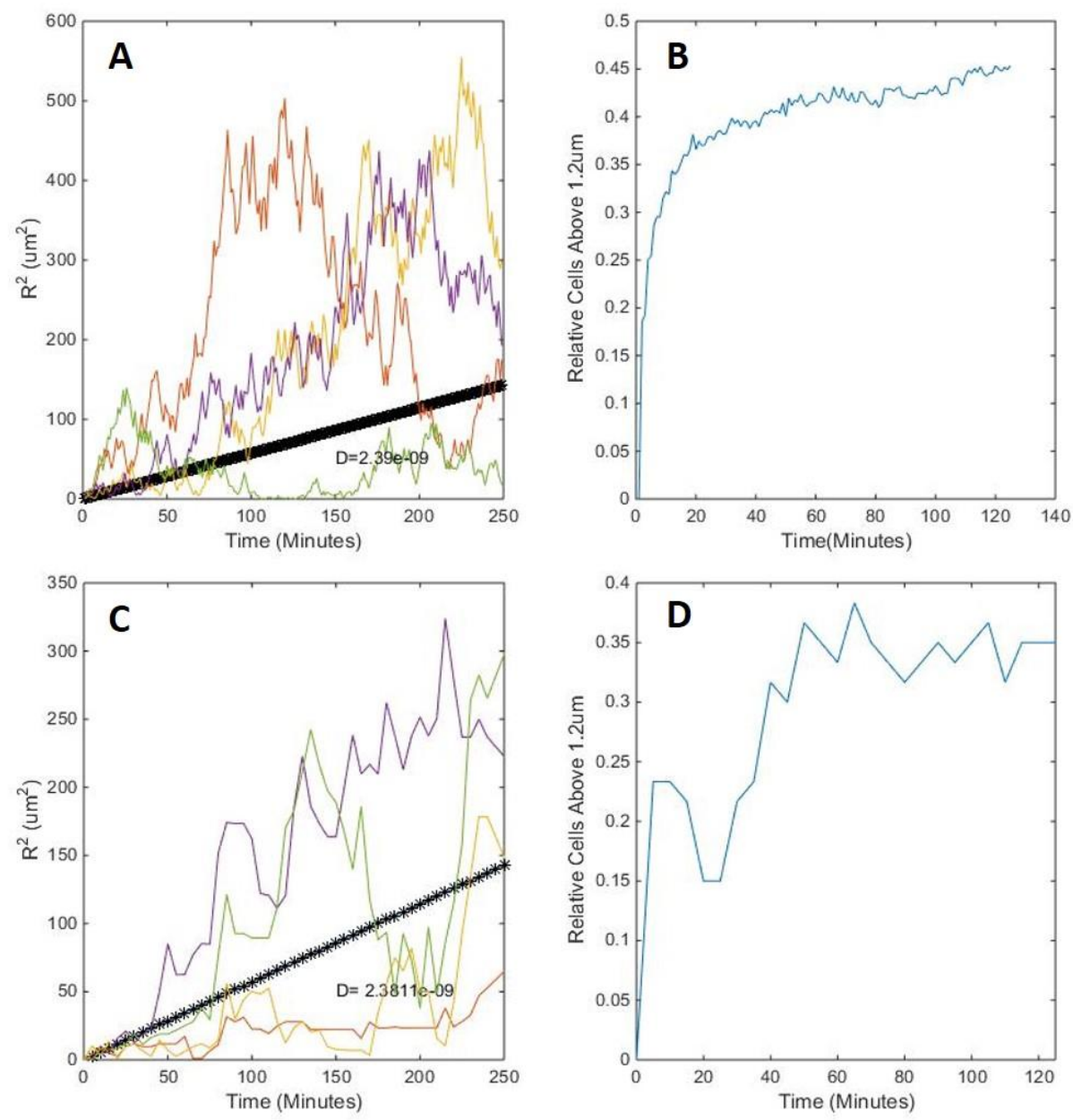

Figure 7| Random motility validation with experimental data.

Computational agent model output for random cancer cell motility (top panel) versus experimentally determined data for cancer cell motility in the absence of attractant (bottom panel). (A/C) Squared displacement from the origin relative to time for four representative cancer cells and average mean squared displacement (solid black line) for all cells (1000 model, 60 experimental). (B/D) The relative cells (cells/total cells) above $1.2 \mathrm{um}$ in the positive y direction.

\subsubsection{Mechanotransduction and autologous chemotaxis mediate flow responsive invasion}

After validation of random motility, blocking studies were performed both in vitro and in silico to simulate disruption of specific receptors (CD44/CXCR4) and the specific ligand (CXCR4) hypothesized to be important in flow stimulated invasion. Migration simulations were performed using the percentages 
of expected cell types based on flow cytometry data (Figure 8A). Simulated invasion and experimental invasion results were comparable (Figure 8B).

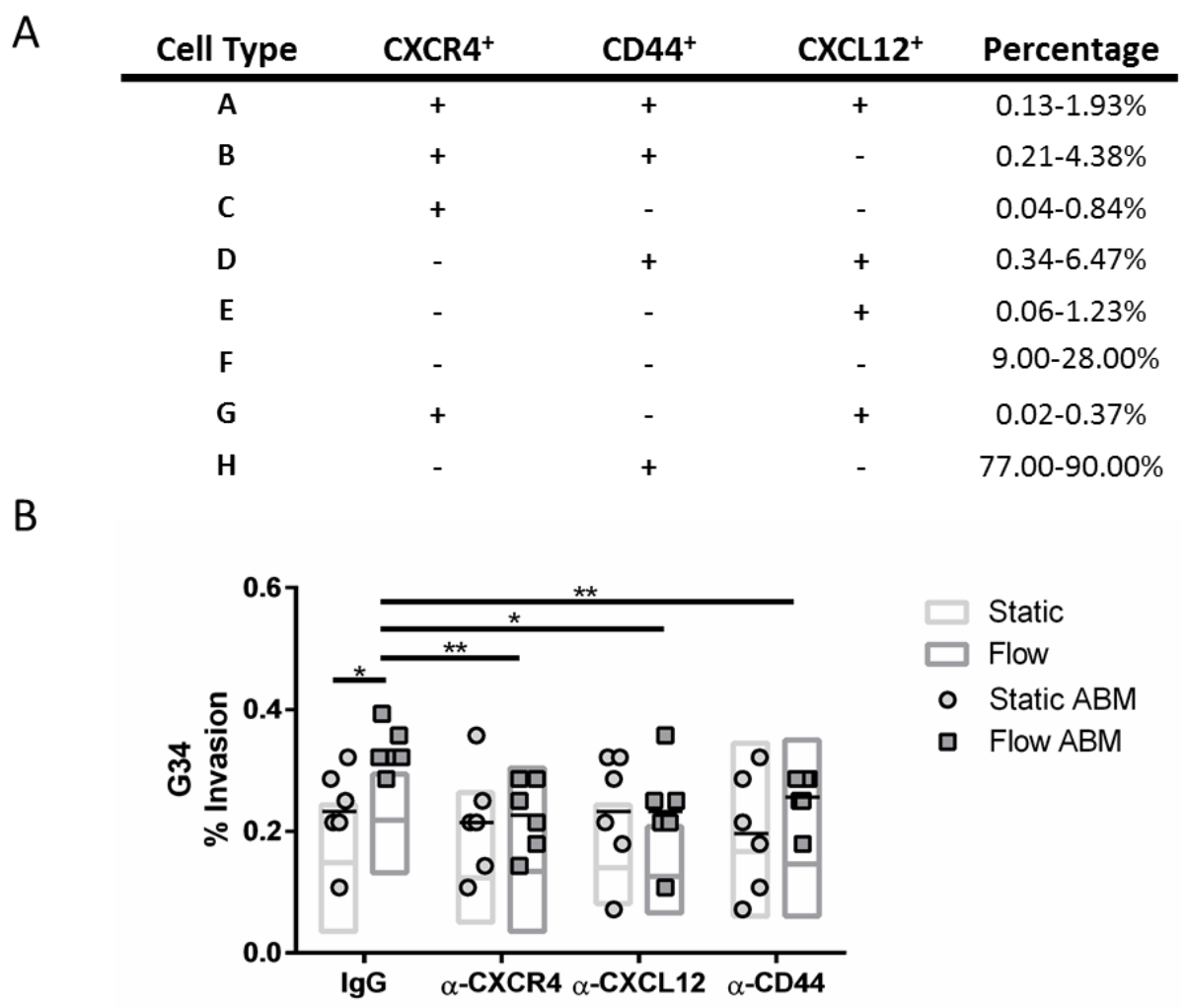

\section{Figure 8| Development of an agent-based model predicts flow-stimulated invasion response to blocking in G34.}

(A) Representative ranges for single and double positive populations of receptor and ligand positive cells in G34 as based on experimental flow cytometry data. (B) Agent-based model output for G34 compared with experimental data with blocking. Significance is for agent-based model output by unpaired t-tests with $\mathrm{n}=6$ independent simulation runs. ${ }^{*} \mathrm{p}<0.05,{ }^{*} \mathrm{p}<0.01$ [Figure Modified from Kingsmore et. al].

Notably, the G34 cell line was flow responsive, as the invasion of the cells increased with introduction of interstitial flow; however, blocking of either CD44, CXCL12, or CXCR4 reduced this response to insignificant levels as compared to the static control. Model simulations confirmed this result. This implies that flow responsive invasion in this particular cell line is likely mediated both by CD44 facilitated mechanotransduction and CXCR4-CXCL12 migratory signaling, as blocking of either of these mechanisms removed the flow response. Neither mechanism was sufficient to elicit flow responsive invasion alone, but taken together, the effect is observe. It was further hypothesized that autologous 
chemotaxis was the major effector of this increased invasion in relation to the CXCR4/CXCL12 receptorligand paired signaling.

\subsubsection{Two subpopulations account for increased motility in the presence of interstitial flow}

Since it was hypothesized that autologous chemotaxis and mechanotransduction were both necessary for flow responsive invasion, a sensitivity analysis of the $\mathrm{ABM}$ was performed to test the

importance of specific cell types on the overall invasion response. Specifically, the cell types of relevance to autologous chemotaxis (CXCR4+/CXCL12+, Figure 9A/B) and increased CD44 phosphorlyation due to receptor coupling (CD44+/CXCR4+, Figure 9C/D) were manipulated in silico (Figure 9 E/F). 

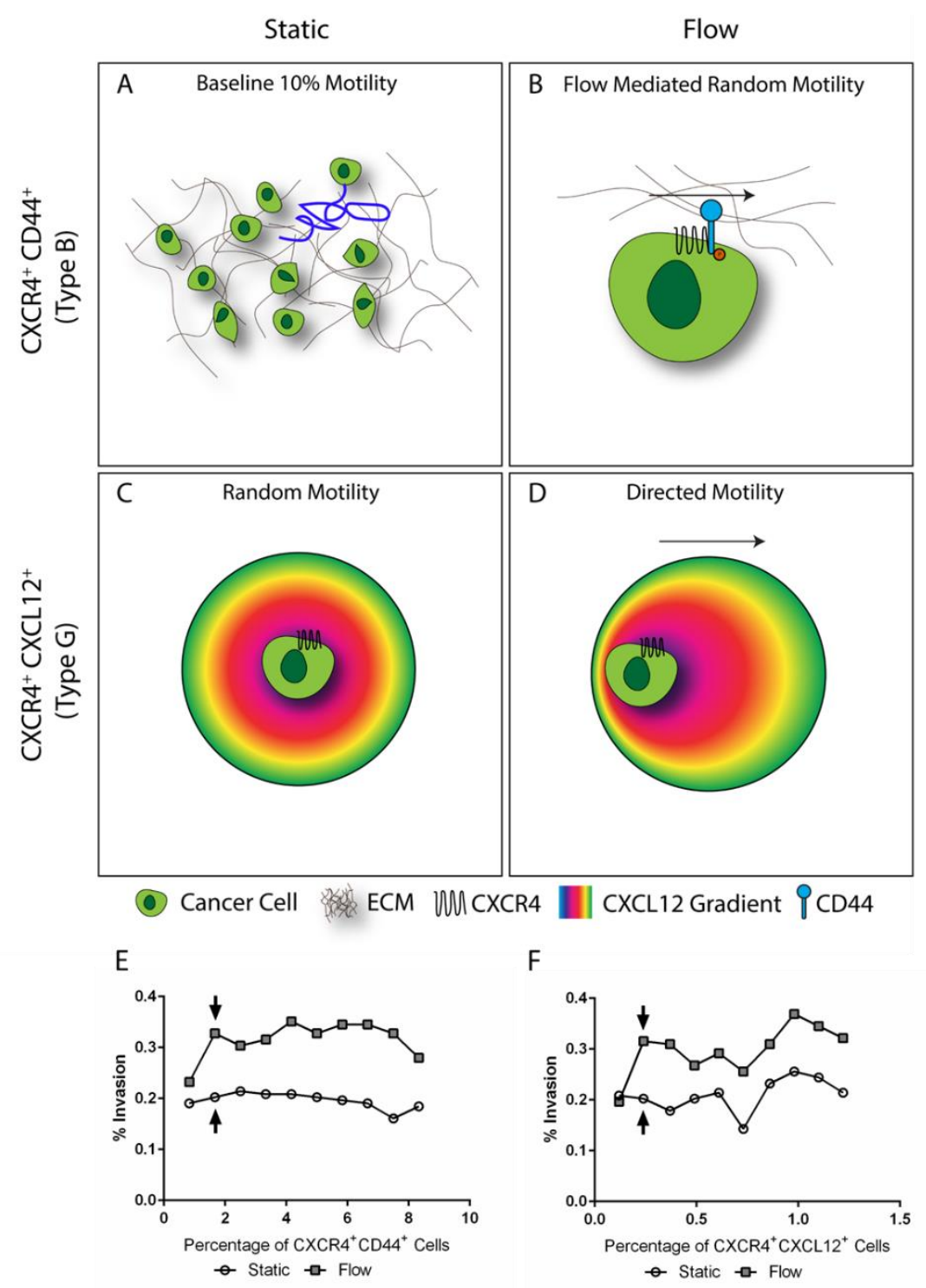

Figure 9| Sensitivity analysis of ABM indicates the importance of cell type subpopulations. Description of ABM motility mechanisms for CXCR4 ${ }^{+} \mathrm{CXCL} 12^{+}$with (A) and without (B) simulated interstitial flow and $\mathrm{CXCR} 4^{+} \mathrm{CD} 44^{+}$populations with $(\mathbf{C})$ and without (D) simulated interstitial flow. (E) Sensitivity analysis of CXCR $4^{+} \mathrm{CXCL} 12^{+}$populations ( $\mathrm{n}=6$ simulation runs). Arrows indicates baseline percentage $(0.24 \%)$ (F) Sensitivity analysis of CXCR4 ${ }^{+} \mathrm{CD} 44^{+}$ populations ( $\mathrm{n}=6$ simulation runs). Arrows indicates baseline percentage (1.67\%). Data shown are mean \pm SEM. static flow lines significant $p<0.001$ by MANOVA.

Baseline percent expression of Type $\mathrm{G}\left(\mathrm{CXCR} 4^{+} \mathrm{CXCL} 12^{+}\right)$cells was $0.15-0.34 \%$. Baseline percent expression of Type B $\left(\mathrm{CXCR} 4^{+} \mathrm{CD} 44^{+}\right)$cells was $1.30-2.03 \%$. Static and flow curves were significantly different $(\mathrm{p}<0.001)$. A decrease in the percentage of either cell type was sufficient to mitigate flow 
responsive invasion. A single percentage point increase in the population of CXCR $4^{+} \mathrm{CXCL} 12^{+}$cells causes larger increase in invasion than a single percent increase in the $\mathrm{CXCR} 4{ }^{+} \mathrm{CD} 44^{+}$population.

\subsection{Discussion and Conclusion}

\subsubsection{Subpopulation protein expression mediates flow responsive invasion}

Replication of blocking in vitro interstitial flow studies with a comparable agent-based model of cellular migration and invasion indicates that the increase in invasion under flow conditions is mediated by two separate mechanisms, autologous chemotaxis, previously described by Munson et al. ${ }^{55}$, and CD44 mechanotransduction. Blocking of either CD44 or CXCR4 in vitro was sufficient to remove increased invasion with flow, which indicated that both proteins mediate flow responsive invasion, however, the extent of flow responsive invasion is mediated by the stochasticity of the system.

The importance of autologous chemotaxis was confirmed by sensitivity analysis. Reduction of $\mathrm{CXCR}^{+} / \mathrm{CXCL} 12^{+}$cell populations removed increased flow responsive invasion. This indicates that autologous chemotaxis is a major effector of the invasion response. A comparable decrease in the $\mathrm{CD}_{4} 4^{+} / \mathrm{CXCR} 4^{+}$population also removed flow responsive invasion effects. This could indicated that phosphorylation of the CXCR4 receptor in response to ECM mediated mechanotransduction by CD44, as described by Rousseau ${ }^{57}$, could be responsible for the increased invasion with flow. Additionally, the sensitivity analysis indicates that autologous chemotaxis dominates this effect as compared to mechanotransduction, as a single percentage point increase in the population of CXCR $4^{+} \mathrm{CXCL} 12^{+}$cells causes larger increase in invasion than a single percent increase in the $\mathrm{CXCR} 4^{+} \mathrm{CD} 44^{+}$population.

\subsubsection{Conclusion}

An agent-based model of glioma cell invasion was developed using the methods described by others, and its utilization confirms the propriety of agent-based models utilizing motility algorithms. Random motility was validated using established equations of cellular motility. Incorporation of both directed motility and random motility rules into the agent-based model elucidated the importance of both 
mechanisms to increased glioma invasion in the presence of interstitial flow. Results indicate that two important subpopulations could be driving this response, and selection of either population could increase overall likelihood of metastatic spread. 


\section{CHAPTER 4: An Agent-Based Model of the Tumor Border Transitional Microenvironment}

\subsection{Introduction}

Chemotherapy is still a near-ubiquitous treatment approach for multiple forms of solid tumor cancers. Though it works in many cases, chemotherapy often fails against cancer, resulting in poor prognosis across multiple forms of the disease. Doxorubicin (DOX) is a commonly used chemotherapy against multiple cancers including breast, bladder, ovarian, and lung, alone or in concert with other treatments. ${ }^{82-84}$ Doxorubicin is a common chemotherapeutic delivered prior to, or after surgery in cases of the deadliest form of breast cancer, triple negative. In addition to its clinical relevance, as a drug with wellunderstood pharmacokinetics, doxorubicin provides an optimal model drug for probing dynamics of chemotherapeutic treatment.

Systemic chemotherapeutic delivery has been limited, first and foremost, by transport restrictions to and within tumors ${ }^{32}$. Specifically, the tumor microenvironment (TME), defined as the tumor cells, tumor-associated cells, extracellular matrix, and biomechanical forces ${ }^{1,2}$, that interact in and around the tumor, provides two distinct barriers to drug delivery. Firstly, delivery of chemotherapeutic agents to the tumor bulk from the circulation is attenuated by interstitial fluid pressure, which is elevated in tumors. ${ }^{85}$ This pressure can limit the transvascular movement of small molecule drugs into the interstitial spaces leading to retention of therapy, particularly larger molecules, near the tumor-associated blood vessels. Drug that does move into the interstitial tumor space is subject to a number of further constraints that limit the transport through the tissue. Limited transport of therapeutic agents results from matrix deposition and crosslinking by cancer associated fibroblasts, uptake by therapeutics by stromal cells, and reduced overall void space due to unrestricted cell growth ${ }^{1}$. This limited drug distribution is thought to particularly reduce therapeutic access to invading cancer cells at the tumor border. These cells are especially deadly as they are thought to be responsible for subsequent metastasis of cancers, which is a leading cause of death. Interestingly, at these border regions of the tumors, where cells are invading, there is increased interstitial 
fluid flow. The higher interstitial fluid pressure in the tumor bulk relative to the normal pressure in the surrounding stroma yields an efflux of fluid from the tumor to the surrounding tissue ${ }^{76}$. These forces are known to alter cellular invasion, promoting movement towards draining lymphatics. However, the interaction of these particular forces with chemotherapeutic transport and delivery is not explored in the context of this complex transitional microenvironment.

Secondly, intercellular interactions in the tumor microenvironment can contribute to reduced therapeutic response. Several TME-mediated factors have been implicated in the development of chemotherapy resistance within solid tumors ${ }^{86}$. These factors include hypoxia ${ }^{87}$, reduced $\mathrm{pH}^{88}$, nutrient deprivation $^{89}$, adhesion-mediated resistance ${ }^{90,91}$, and drug gradient formation ${ }^{37}$. However, cancer-stromal interactions, specifically between cancer cells and fibroblasts in breast cancer, appear to be a dominating factor in acquired chemotherapy resistance ${ }^{92}$. Cancer associated fibroblasts secrete pro-survival factors that limit cancer cell apoptosis and could act to protect cancer cells from chemotherapeutic effects ${ }^{20}$. In the context of neoadjuvant breast cancer therapy, the presence of cancer associated fibroblasts signatures has been directly implicated in poorer survival outcomes ${ }^{43}$, and targeted therapy against these stromal cells has been shown to improve intratumoral uptake of doxorubicin within in vivo murine models ${ }^{44}$.

In relation to these barriers to chemotherapy treatment, the tumor border is a unique environment, as stromal interactions, chemotherapy gradients, and interstitial flow are all present in this region. Interstitial fluid velocity and pressure are the greatest at the tumor border ${ }^{29}$, thus mediating convectiondriven chemotherapy transport through this region ${ }^{35}$. Additionally, gradients of doxorubicin have been observed at the breast tumor border in $v i v o^{37}$, providing a potential for adaptive chemotherapy resistance. Finally, cancer cell propinquity to stromal fibroblasts, coupled with zones of cellular transition ${ }^{93}$ from the tumor to the surrounding stroma, yields stromal heterogeneity. At the tumor border, the tissue transitions from regions of very few fibroblasts in the tumor bulk to regions of very few cancer cells relative to stromal cells; therefore, this transition region is defined by cellular gradients, which could mediate further cancer cell insensitivity to chemotherapy due to fibroblast interactions. Collectively, this distinct environment 
couples several TME-specific factors that could lead to chemotherapy resistance and decreased patient survival.

Due to the complexity of this region, robust in vitro and in silico models are necessary to probe the effects of therapy coupled with interstitial flow. 3D collagen hydrogels have been utilized in several cancer contexts as in vitro models of the tumor microenvironment ${ }^{76}$. These models can incorporate extracellular matrix proteins and pressure driven flow, thus replicating biophysical parameters inherent to in vivo tumors and providing a culture medium that is more replicative of real tissues than $2 \mathrm{D}$ culture systems ${ }^{94}$. In silico research methodologies provide a supplementary data analysis context that can enhance and predict biological phenomena. Agent-based models (ABMs) are particularly suited for biological applications, as they can predict and describe complex spatial and temporal biological interactions ${ }^{65,66}$.

Here, we establish in vitro and in silico models that elucidate the effects of fluid and solute transport, cellular heterogeneity, and fibroblast interactions on breast cancer viability following doxorubicin treatment. Our study utilizes a novel 3D in vitro tumor bulk to stroma transition model (TSTM), as well as concurrent 2D culture systems, to predict the regional variations in viability that occur within the microenvironment at the tumor border. Additionally, in silico methodologies predict the dominant fluid dynamic properties that influence doxorubicin treatment efficacy within the tumor border transitional region. Together our data provide evidence of a unique fibroblast protective effect that yields varied resistance to chemotherapy in a cancer to fibroblast ratiometric dependent manner, which is sustained in several experimental models. These findings illuminate the importance of regional TME heterogeneity in selecting for viable populations that could be impacting breast cancer progression.

\subsection{Materials and Methods}

\subsubsection{Cell Culture}

MDA-MB-231 human breast adenocarcinoma (Tumor cells: TCs) and human dermal fibroblasts (Fibroblasts: Fbs) were obtained from the American Type Culture Collection (ATCC). Both cell types were cultured in Dulbecco's modified Eagle's medium (DMEM, Gibco), supplemented with 10\% fetal bovine 
serum (FBS, Seradigm). Cells were passaged weekly and grown at $37^{\circ} \mathrm{C}$ in a sterile incubator $(5 \% \mathrm{CO} 2$ and $95 \%$ Oxygen) in gamma irradiated tissue culture treated flasks.

\subsubsection{Two-Dimensional (2D) Conditioned Media Assay}

Fb-conditioned media was created by incubating fibroblasts in cell culture flasks with full (DMEM+10\%FBS) media for 24 hours. Control media was collected from a second flask incubated simultaneously, in the absence of cells. MDA-MB-231 cells were seeded into a 48 well tissue culture treated plate at a density of 20,000 cells per well into either $\mathrm{Fb}$-conditioned or unconditioned media. After 24 hours, the media was replaced with $10 \mathrm{uM}$ of doxorubicin $\mathrm{HCl}$ (Fisher Scientific) in serum free media. TC percent live and doxorubicin accumulation were determined as described.

\subsubsection{Two-Dimensional (2D) Hanging Well Coculture Assay}

MDA-MB-231 cells were seeded into a 24 well tissue culture companion plate for hanging cell culture inserts (VWR International) at a density of 40,000 cells per well. HDFs were seeded into hanging culture inserts (VWR international), with 1.0 micron membrane pore size, at a density of 10,000 cells per insert. For the control condition, HDF seeding was neglected. Cells were grown in serum free DMEM for 24 hours. Prior to introduction of chemotherapy, the cell culture insert was removed for the conditioned experimental group. Doxorubicin was introduced, to bring the final concentration to $10 \mathrm{uM}$. TC percent live and doxorubicin accumulation were determined as described.

\subsubsection{Two-Dimensional (2D) Ratiometric Coculture Assay}

\section{Constant Total Cell Number Ratio Experiment}

For the first experiment, HDFs and Cell Tracker (ThermoFisher) deep red labelled MDA-MB-231 cells were introduced into the same well of a 48 well tissue culture treated cell culture plate at varied ratios of cancer cells to fibroblasts $(4: 1,2: 1,1: 1,1: 2,1: 4)$. Here, the total cell density was held constant at 30,000 cells, and cell numbers were varied internally. For example, a ratio of 4:1 had 24,000 cancer cells and 6,000 
fibroblasts, while a ratio of 2:1 had 20,000 cancer cells and 10,000 fibroblasts. Control conditions were seeded at cancer cell densities comparable to experimental conditions, however, fibroblasts were not introduced. The cells were incubated for 24 hours at $37^{\circ} \mathrm{C}$, followed by introduction of doxorubicin HCL diluted in serum free DMEM (10uM).

\section{Constant Total Tumor Cell Number Ratio Experiment}

For the second experiment, HDFs and Cell Tracker (ThermoFisher) deep red labelled MDA-MB231 cells were also introduced into the same well of a 48 well tissue culture treated cell culture plate at varied ratios of cancer cells to fibroblasts $(4: 1,2: 1,1: 1,1: 2,1: 4)$. Here, the cancer cells were kept constant at 10,000 cells while the fibroblast seeding varied. For example, a ratio of 4:1 had 10,000 cancer cells and 2,500 fibroblasts, while a ratio of 2:1 had 10,000 cancer cells and 5,000 fibroblasts. MDA-MB-231 cells were seeded at 10,000 cells per well without fibroblasts, for control comparison. The cells were incubated for 24 hours at $37^{\circ} \mathrm{C}$, followed by introduction of doxorubicin HCL diluted in serum free DMEM (10uM).

\subsubsection{Two-Dimensional (2D) Live/Dead Analysis}

Following 6 hours of doxorubicin treatment $(10 \mathrm{uM})$ and subsequent 24 hour incubation in serum free DMEM, cells were incubated with NucBlue live cell stain (Life Technologies) and NucGreen Dead 488 cell stain (Life Technologies) in serum free DMEM for 20 minutes at room temperature. Each experimental well was imaged using fluorescence microscopy (EVOS FL). Live and dead cells were counted using the ImageJ cell counter plugin (NIH). For co-culture assays, cancer cells were identified by the presence of the CellTracker Deep Red dye prior to assessment for live/dead. For all assays, five images were taken per experimental technical replicate ( $n=3$ per experiment) and averaged for statistical analysis.

\subsubsection{Doxorubicin Accumulation Analysis}

For doxorubicin accumulation experiments, the cells were trypsinized ( $0.25 \%$ trypsin, Gibco) at two hour time points (at 6 hours post doxorubicin introduction for the 2D hanging well experiments), 
centrifuged at $2000 \mathrm{rpm}$ for two minutes, and subsequently lysed with RIPA Buffer (Thermo Scientific) for 15 minutes on ice. Cellular lysate fluorescence intensity was determined using a fluorescent plate reader (Omega FLUOstar) at 495nm excitation and 590nm emission. Fluorescence intensity was compared to a doxorubicin standard curve for concentration determination.

\subsubsection{Three-Dimensional (3D) Homogenous In vitro Interstitial Flow Model}

50ul of rat tail collagen I (Corning) and basement membrane extract (Trevigen) $(1.8 \mathrm{mg} / \mathrm{ml}$ Collagen, $0.5 \mathrm{mg} / \mathrm{ml}$ BME), containing cell tracker deep red dye (ThermoFisher) labelled MDA-MB-231 cells and HDFs at varied ratios (TC alone, 4:1, 1:1, 1:4) of cancer cells to fibroblasts, was added into a 96well tissue culture insert (Corning). Total cell density in the gels was 100,000 total cells/mL. Hydrogels were crosslinked at $37^{\circ} \mathrm{C}$ for 30 minutes. Afterward, the gels were rehydrated with a drop of serum free media and placed in an incubator for 3 hours, to allow for cell adhesion within the matrix. After three hours, serum free DMEM was added to the bottom compartment of the insert and either serum free DMEM (control condition) or 10uM doxorubicin diluted in basal DMEM was added onto the top of the gel. Gravity driven flow ( 0.5 microns/s) was introduced for 18 hours, at which point the media was removed, and the gels were flushed with basal media for a comparable amount of time. The basal media was replaced with NucBlue live cell stain (Life Technologies) and NucGreen Dead 488 cell stain (Life Technologies) in serum free DMEM and incubated for one hour at $37^{\circ} \mathrm{C}$.

The gels were removed from the inserts and imaged using fluorescent microscopy (EVOS FL). Five images were taken per experimental technical replicate and averaged for statistical analysis. Dead cancer cells were determined by colocalization of blue, deep red, and green fluorescent markers.

\subsubsection{Three Dimensional (3D) In vitro Tumor to Stroma Transition Hydrogel Model}

Five separate solutions of rat tail collagen I (Corning) and basement membrane extract (Trevigen) $(1.8 \mathrm{mg} / \mathrm{mL}$ collagen, $0.5 \mathrm{mg} / \mathrm{ml} \mathrm{BME})$ containing cell tracker deep red dye (Thermo Fisher) labelled MDA-MB-231 cells and HDFs at varied ratios (4:1, 2:1, 1:1, 1:2, 1:4) of cancer cells to fibroblasts were 
created. Each solution had a total cell density of 100,000 total cells $/ \mathrm{ml}$. $50 \mathrm{uL}$ of successive ratios starting with 1:4 were introduced into a tissue culture insert (Corning) with 20 minutes of crosslinking at $37^{\circ} \mathrm{C}$ between the addition of each new layer. After the final layer was added, the entire gel was crosslinked at $37^{\circ} \mathrm{C}$ for 45 minutes. For the single culture layered gels, the same procedure was followed, however, cancer cells were seeded in the gels in the absence of HDFs at densities comparable to the experimental MDAMB-231 densities.

Basal media was introduced into the bottom of the cell culture insert. Either basal media or 10uM doxorubicin solution was added to the top of the hydrogel. Fluid flowed through the gel $(\sim 0.5$ microns/s) for 18 hours. The media was removed and the gels were flushed with basal media for 18 hours. Live/dead fixable green dye (Life Technologies) in PBS was flushed through the gel for one hour. Afterward, the gels were fixed with $4 \%$ paraformaldehyde for 18 hours at $4{ }^{\circ} \mathrm{C}$. The hydrogels were removed from the inserts and stained with DAPI diluted in PBS on a shaker for 1 hour. Gels were imaged using confocal fluorescent imaging (Zeiss 700). Z-stacks were developed with 25 slices through the gel. Dead cancer cells were determined by colocalization of green, deep red, and glue fluorescent markers. The dead cells in five successive slices were averaged for each technical replicate.

For imaging of the gel prior to introduction of flow, the cancer cells in the top, middle, and bottom layer were labelled with cell tracker deep red (Thermo Fisher), while all other cancer cells were labelled with cell tracker green (Thermo Fisher). The gels were immediately fixed with $4 \%$ paraformaldehyde at $4^{\circ} \mathrm{C}$ for 18 hours, following crosslinking. The gels were removed from the inserts and stained with DAPI diluted in PBS on a shaker for 1 hour. Gels were imaged using confocal fluorescent imaging (Zeiss LSM 700). Z-stacks were developed with 200 separate slices.

\subsubsection{Generation of IC50 Curves}

HDFs and MDA-MB-231 cells were seeded into separate 96 well tissue culture plates at a density of 10,000 cells per well. Cells were incubated for 24 hours at $37^{\circ} \mathrm{C}$. Afterward, doxorubicin solutions at 
varying concentrations diluted from $100 \mathrm{uM}$ were introduced into appropriate wells. Dead cells were determined as previously described. IC50 values were determined using Matlab curve fitting algorithms.

\subsubsection{Development of Concentration Gradients using Comsol}

Spatial concentration profiles were developed using a finite element based, partial differential boundary solver in Comsol Multiphysics. 2D Geometry of the hydrogel spatial grid (4.5 $\mathrm{mm}$ by $3.5 \mathrm{~mm})$ was determined by the volume of hydrogel introduced into the cell culture insert and the cell culture insert dimensions provided by the manufacturer.

Time dependent convection within the simulated hydrogel space was defined by Darcy's law of fluid dynamics, which has previously been applied to interstitial fluid flow in hydrogels and tumors ${ }^{95}$. Darcy's law describes volumetric flow rate $\left(Q_{m}\right)$, as

$$
Q_{m}=\frac{\partial}{\partial t}\left(\varepsilon_{p} \rho\right)+\nabla *(\rho u)
$$

where $\varepsilon_{p}$ (unitless) is the porosity coefficient of the matrix, $\rho\left(\frac{\mathrm{kg}}{\mathrm{m}^{3}}\right)$ is the fluid density and $u\left(\frac{m}{\mathrm{~s}}\right)$ is the average velocity within the simulated space. Porosity was determined from specific collagen content and the collagen concentration within the hydrogels as described by Ramanujan et. al. (Table 2). The velocity in the hydrogel is further described by

$$
u=\frac{-K}{\mu} \nabla p
$$

where $K\left(\mathrm{~m}^{2}\right)$ is the matrix permeability coefficent, $\mu(\mathrm{Pa} * s)$ is the dynamic viscosity of the fluid, and $p(P a)$ is the pressure. The permeability coefficient (Table 2) was determined experimentally using a similar relationship

$$
K=\frac{Q}{A} *\left(\frac{L}{\Delta p}\right) * \mu
$$

where $Q\left(\frac{m^{3}}{s}\right)$ is the volumetric flow rate through the gel, $A\left(m^{2}\right)$ is the cross-sectional area of the gel, $L(m)$ is the height of the gel, and $\Delta p(\mathrm{~Pa})$ is the difference in pressure between the top and the bottom of the gel. 
Concentration profiles at various times using the general diffusion convection differential equation

$$
\frac{\partial c}{\partial t}+\nabla *\left(-D_{i} \nabla c_{i}\right)+\mathrm{u} *\left(\nabla c_{i}\right)=R_{i}
$$

where $c\left(\frac{m o l}{m^{3}}\right)$ is the concentration of drug, $D_{i}\left(\frac{m^{2}}{s}\right)$ is the diffusion coefficient, $\mathrm{R}$ is the reaction term, assumed to be zero, and $u\left(\frac{m}{s}\right)$ is the Darcy velocity determined previously. The diffusion coefficient was determined from literature representation of diffusion within low collagen percentage hydrogels normalized with a hydrodynamic radius of $2 \mathrm{~nm}$ (Table 1 ).

Table 2| Comsol modeling parameters

\begin{tabular}{|c|c|c|c|}
\hline Parameter & Symbol & Value & Source \\
\hline Permeability & $K$ & $3.19 \mathrm{e}^{-14}\left[\mathrm{~m}^{\wedge} 2\right]$ & Experimental \\
\hline Porosity & $\varepsilon_{p}$ & 0.997 [Dimensionless] & {$[95]$} \\
\hline $\begin{array}{l}\text { Diffusion } \\
\text { Coefficient }\end{array}$ & $D$ & $6 \mathrm{e}^{-11} \quad\left[\mathrm{~m}^{\wedge} 2 / \mathrm{s}\right]$ & [95] \\
\hline $\begin{array}{l}\text { Time Dependent } \\
\text { Fluid Volume }\end{array}$ & $V$ & $V=\beta_{1}+\left(\frac{\beta_{2}-\beta_{1}}{1+\frac{\beta_{3}}{t}}\right)^{\beta_{4}}[\mu \mathrm{L}]$ & Experimental \\
\hline $\begin{array}{l}\text { Curve Fitting } \\
\text { Parameter }\end{array}$ & $\beta_{1}$ & $37.94[\mu \mathrm{L}]$ & Experimental \\
\hline $\begin{array}{l}\text { Curve Fitting } \\
\text { Parameter }\end{array}$ & $\beta_{2}$ & $84.56[\mu \mathrm{L}]$ & Experimental \\
\hline $\begin{array}{l}\text { Curve Fitting } \\
\text { Parameter }\end{array}$ & $\beta_{3}$ & $1.09[\mathrm{hr}]$ & Experimental \\
\hline $\begin{array}{l}\text { Curve Fitting } \\
\text { Parameter }\end{array}$ & $\beta_{4}$ & 4.00 [Dimensionless] & Experimental \\
\hline Fluid Density & $\rho$ & $998 \quad\left[\frac{\mathrm{kg}}{\mathrm{m}^{3}}\right]$ & Known \\
\hline Dynamic Viscosity & $\mu$ & $9.79 \mathrm{e}^{-4}[P a * s]$ & Known \\
\hline
\end{tabular}

\subsubsection{Agent-based Model Construction}

Comsol time-dependent convection diffusion concentration gradients (Comsol Multiphysics 5.1) were developed using Darcy's law of fluid flow coupled with simple diffusion dynamics, representative of 
doxorubicin transport within in vitro breast mimetic collagen hydrogel/BME hydrogels ${ }^{95}$ (Supplemental Methods). Physical model parameters were determined from experimental methods and literature values (Table 3). Concentration profiles were outputted as text files for use in agent-based model (ABM) simulations (Figure 10a).

Table 3| Agent-based model equations and parameters

\begin{tabular}{|c|c|c|c|c|}
\hline Parameter & Cell Type & Equation & Units & Bounds/Variables \\
\hline 2D IC50 Percent Live & MDAMB-231 & plive $=\beta_{M 1}+\left(\frac{\beta_{M 2}-\beta_{M 1}}{1+\frac{\beta_{M 3}}{D o s e}}\right)^{\beta_{M 4}}$ & $\%$ & $\begin{array}{c}\beta_{M 1}=78.11 \% \\
\beta_{M 2}=-0.48 \% \\
\beta_{M 3}=3.66 \mu \mathrm{M} \\
\beta_{M 4}=1.09[\text { Dimensionless }]\end{array}$ \\
\hline $\begin{array}{l}\text { 2D Cellular Density } \\
\text { Dependent Percent live }\end{array}$ & MDAMB-231 & $\begin{array}{c}\text { plive }=0.0003 * \text { Density }+34.484 \\
\text { plive }=41.68 \\
\text { plive }=36.28\end{array}$ & $\%$ & $\begin{array}{c}6000 \leq \text { Density } \leq 24000 \\
\text { Density }>24000 \\
\text { Density }<6000\end{array}$ \\
\hline $\begin{array}{c}\text { 2D 231/HDF Ratio } \\
\text { Dependent Percent Live }\end{array}$ & MDAMB-231 & $\begin{array}{c}\text { plive }=-2.50 * \text { ratio }^{2} \\
+18.77 * \text { ratio }+41.054 \\
\text { plive }=76.38 \\
\text { plive }=43.71\end{array}$ & $\%$ & $\begin{array}{c}0.25 \leq \text { ratio } \leq 4 \\
\text { ratio }>4 \\
\text { ratio }<0.25\end{array}$ \\
\hline 2D IC50 Percent Live & HDF & plive $=\beta_{H 1}+\left(\frac{\beta_{H 2}-\beta_{H 1}}{1+\frac{\beta_{H 3}}{\text { Dose }}}\right)^{\beta_{H 4}}$ & $\%$ & $\begin{array}{c}\beta_{H 1}=74.07 \% \\
\beta_{H 2}=0.04 \% \\
\beta_{H 3}=2.105 \mu \mathrm{M} \\
\beta_{H 4}=1.01[\text { Dimensionless }]\end{array}$ \\
\hline $\begin{array}{c}\text { 2D Cellular Density } \\
\text { Dependent Percent Live }\end{array}$ & HDF & $\begin{array}{c}\text { plive }=-0.0011 * \text { Density }+60.88 \\
\text { plive }=54.28 \\
\text { plive }=34.48\end{array}$ & $\%$ & $\begin{array}{c}6000 \leq \text { Density } \leq 24000 \\
\text { Density }>24000 \\
\text { Density }<6000\end{array}$ \\
\hline $\begin{array}{c}\text { 2D 231/HDF Ratio } \\
\text { Dependent Percent Live }\end{array}$ & HDF & $\begin{array}{c}\text { plive }=-4.30 * \text { ratio }^{2} \\
+29.75 * \text { ratio }+15.82 \\
\text { plive }=66.02 \\
\text { plive }=22.99\end{array}$ & $\%$ & $\begin{array}{c}0.25 \leq \text { ratio } \leq 4 \\
\text { ratio }>4 \\
\text { ratio }<0.25\end{array}$ \\
\hline
\end{tabular}

The agent-based model (ABM) was constructed using the Repast Java framework and the Relogo Java package. A 2D coordinate grid of $64 \times 64$ patches was initiated to represent a $4.0 \mathrm{~mm} \times 3.5 \mathrm{~mm}$ finite slice of experimental gel ${ }^{54}$. For homogenous gel simulations, the total number of cells was 2800 , and the fibroblasts and/or cancer cells were spawned randomly within the gel at the user specified ratios (Figure 10a). For the layered tumor transition model, the grid space was split into 5 equivalent segments (Figure 10c). Fibroblasts and cancer cells were spawned randomly within the sections at user specified ratios for a 
total cell number of 2800 . For the single culture tumor transition model, cancer cells were spawned within segments at densities comparable to experimental conditions. The total cell number was 1400 (Figure 9b).
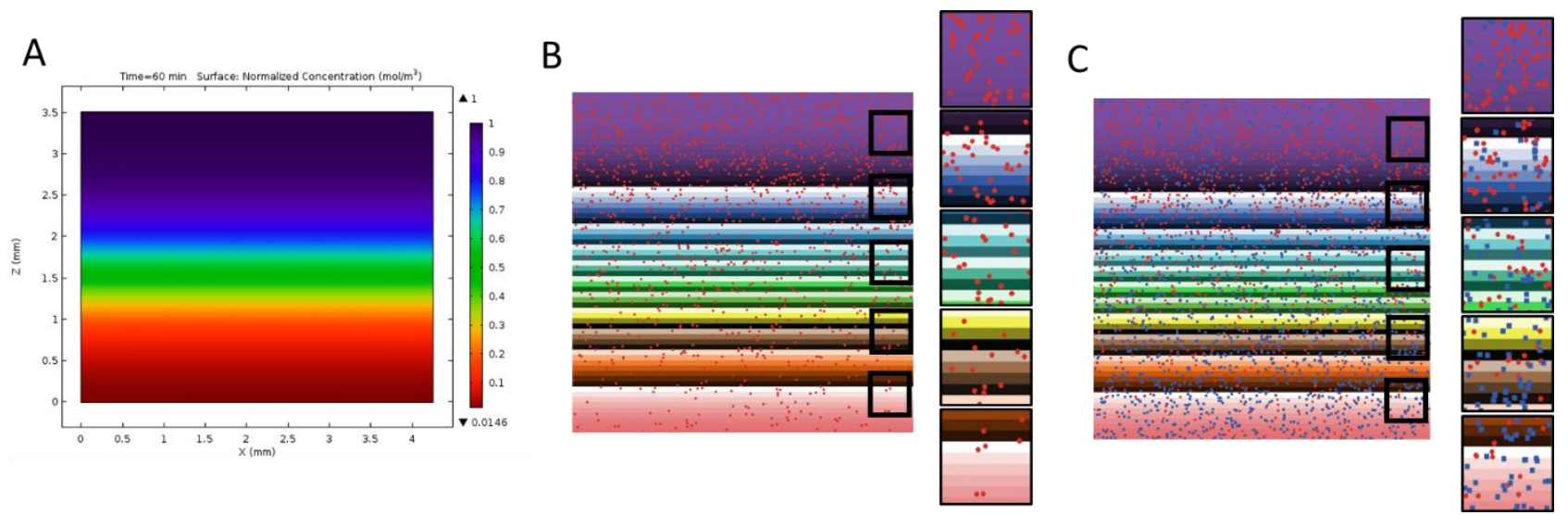

Figure 10| Development of an agent-based model to predict cancer cell response to doxorubicin in 3D tumor bulk to stroma transition environments.

(A) Diffusion-convection concentration gradient developed in Comsol within a simulated 3D hydrogel. (B) The agent-based model incorporates concentration profiles and cancer cells for time dependent cell viability determination. Single culture condition incorporates varied cancer cell numbers decreasing from the top to the bottom of the gel. Fibroblasts are neglected. (C) The agent-based model incorporates concentration profiles, fibroblasts, and cancer cells for time dependent cell viability determination. The coculture condition incorporates varied cancer cell numbers, decreasing from the top to the bottom of the gel and varied fibroblast number decreasing from the bottom to the top of the gel.

Each tic of the scheduling method corresponded to one minute. At each subsequent tic of the scheduling method (Figure 11a), a new concentration profile was parsed and concentration values were stored in appropriate patches. The patches passed these values to corresponding cell(s) located on the patches (Figure 11b). 
A

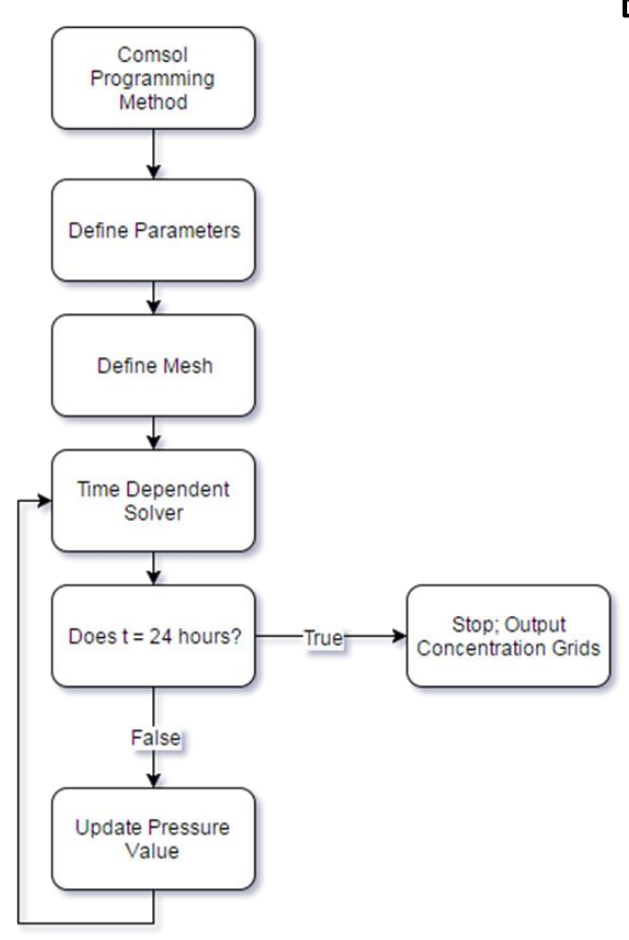

B

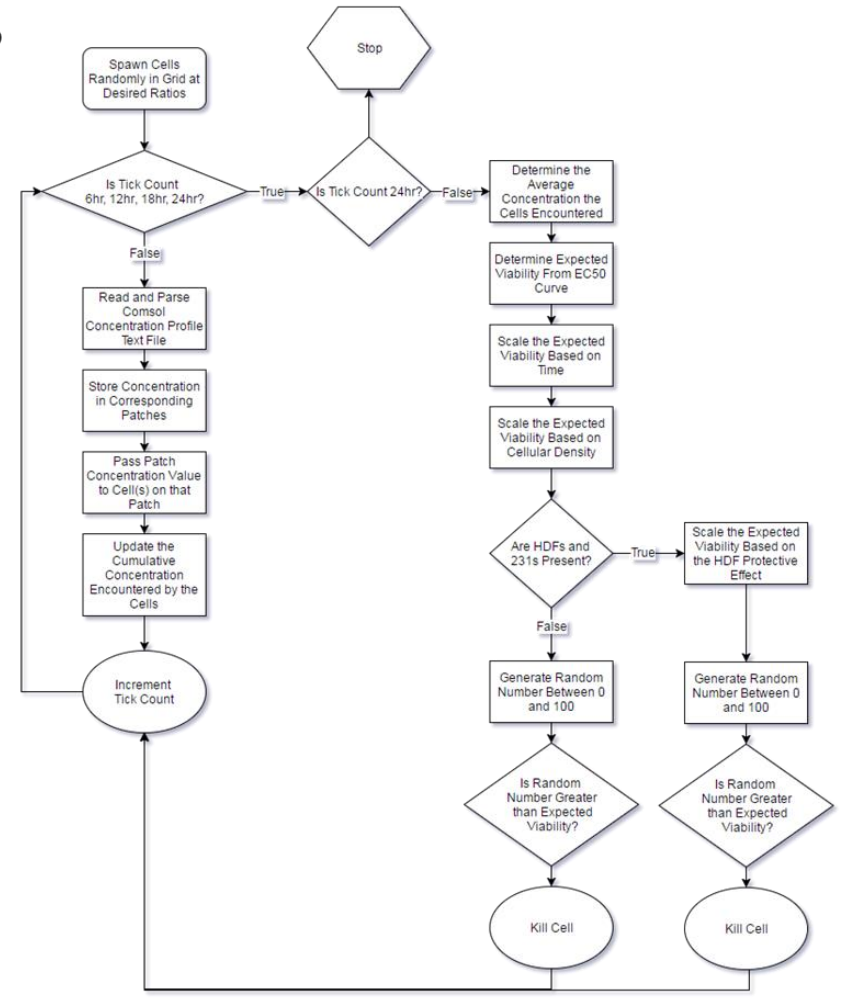

Figure 11| Logic flow charts for in silico modelling.

(A) Comsol programing method for development of spatial and time dependent concentration gradients for use in associated agent-based models. (B) Logic flowchart for agent-based models of doxorubicin treatment within collagen/bme hydrogels incorporating Comsol drug concentration profiles and experimental viability equations (Table 2 ).

The average concentration encountered by the cells was updated based on the stored value. At specified time intervals, the expected live percentage, based on IC50 curves and the average concentration encountered by the cells, was determined. This value was scaled to account for cellular density (Table 2). If the modeling condition accounted for both fibroblast and cancer cells, the expected live percentage was scaled to account for the fibroblast protective effect based on the ratio of cancer cells to fibroblasts. The cells were removed or kept in the simulation after comparing a randomly generated number to the expected live percentage. Percent live of remaining cells was then calculated. 


\subsubsection{Sample Selection}

Patient samples were accessed through the University of Virginia Biorepository and Tissue Research Facility. These samples were selected from patients with a definitive diagnosis of node-negative breast cancer and who received no treatment prior to tumor resection. Samples were de-identified before use. All procedures performed in studies involving human participants were in accordance with the ethical standards of the institutional review board of the University of Virginia and with the 1964 Helsinki declaration and its later amendments or comparable ethical standards.

\subsubsection{Immunohistochemistry}

Formalin-fixed, paraffin-embedded sections were deparaffinized with xylene and rehydrated in graded ethanols and citrate-based antigen retrieval was performed (Vector Labs, Burlingame, CA). Samples were permeabilized (0.01\% Triton) and blocked in goat serum. Based on markers previously established in the literature, breast cancer cells were identified by anti-pan-cytokeratin staining and cancer-associated fibroblasts were identified by anti-alpha-smooth muscle actin staining. Samples were incubated with pancytokeratin antibody (Thermoscientific) followed by secondary Cy5-goat anti-mouse (Thermoscientific). These steps were then repeated for the TRITC-conjugated alpha-smooth muscle actin antibody (ebioscience). The samples were incubated with DAPI (Sigma-Aldrich, St. Louis, MO) and mounted with Fluoromount-G (SouthernBiotech). All antibodies were used at dilutions recommended by the manufacturer for paraffin-embedded tissues. Stained slides were imaged with an EVOS fluorescent microscope (Thermoscientific). Random non-overlapping $856 \times 476 \mu \mathrm{m}(407,465 \mu \mathrm{m} 2)$ regions at the tumor-stroma border were selected for imaging. Images were processed using ImageJ and Photoshop.

Table 4| Antibodies used for immunohistochemical staining.

\begin{tabular}{ccccc}
\hline Antigen & $\begin{array}{c}\text { Antibody } \\
\text { Manufacturer }\end{array}$ & Cat No. & Host & $\begin{array}{c}\text { Concentration } \\
(\boldsymbol{\mu g} / \mathbf{m L})\end{array}$ \\
\hline Pan-Cytokeratin & $\begin{array}{c}\text { Thermo Scientific, } \\
\text { Waltham, MA } \\
\text { Invitrogen, } \\
\text { Waltham, MA }\end{array}$ & MS-343-P0 & $\begin{array}{c}\text { Mouse } \\
\text { Anonoclonal } \\
\text { Goat } \\
\text { polyclonal }\end{array}$ & 4.0 \\
& IgG & & 10.0
\end{tabular}


Alpha-Smooth Muscle

eBioscience,

Waltham, MA

41-9760-80

Mouse

monoclonal

1.0

\subsubsection{Statistical Analysis}

Statistical analyses were run using Graphpad Prism. Paired t-tests and two-way ANOVA were used for analysis of same subject groups. Unpaired t-tests and two-way ANOVA were used for analysis of independent experimental groups and computational data. MANOVA analysis using the SPSS software package was utilized for normalized distance comparisons within experimental gels for both computational and experimental conditions. All assays were performed with a minimum of three biological replicates. $\mathrm{P}<0.05$ was considered statistically significant for all statistical tests.

\subsection{Results}

\subsubsection{Fibroblasts reduce cancer cell death in response to doxorubicin and lowered levels of doxorubicin} accumulation

The effects of contact independent fibroblast signaling on cancer cell response to doxorubicin were first tested by treating MDA-MB-231 cells with either HDF conditioned media or comparable control media (Figure 12A) in 2D for 24 hours. Following doxorubicin treatment, tumor cells grown in conditioned media had a greater percentage of live cells compared to unconditioned controls (Figure 12B). Furthermore, tumor cells treated with either $\mathrm{Fb}$-conditioned or unconditioned media showed no difference in percent live tumor cells in the absence of doxorubicin. Notably, at 2 and 6 hours, the mass of doxorubicin present in cell lysate was significantly decreased following pre-incubation with fb-conditioned media prior to treatment compared to unconditioned media controls (Figure 12C).

To further test contact independent fibroblast signaling, fibroblasts were seeded on a hanging porous insert with tumor cells seeded onto the bottom of the well plate at a ratio of 4:1 tumor cells to fibroblasts (Figure 12D). While preconditioning with fibroblasts showed no effect, co-culturing with fibroblasts during treatment yielded a significant increase in the percentage of live tumor cells compared to 
controls (Figure 12E). The co-culture condition yielded a decrease in cancer cell-internalized mass of doxorubicin compared to the tumor cells alone control (Figure 12F).

A

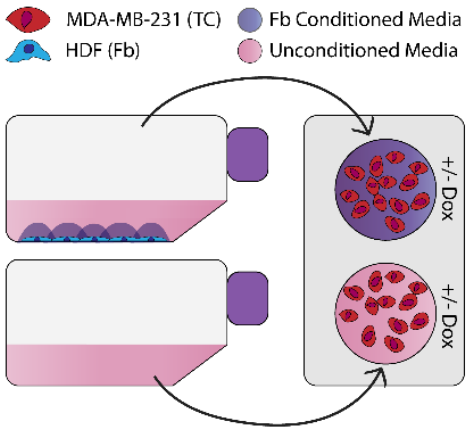

B

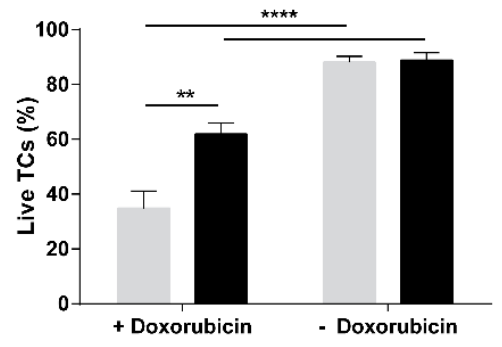

C

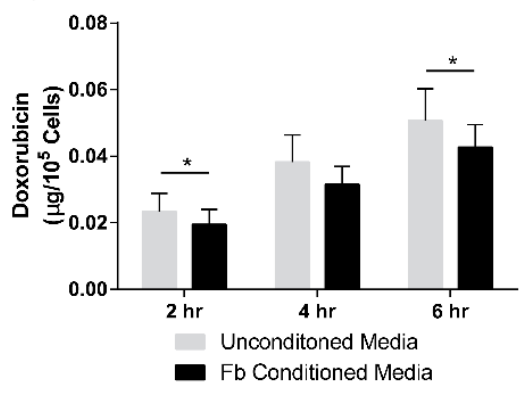

D
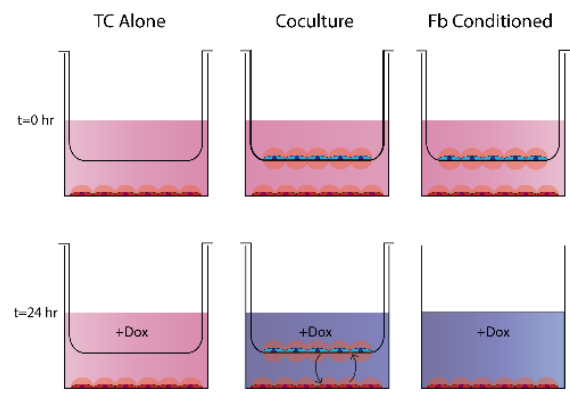

E

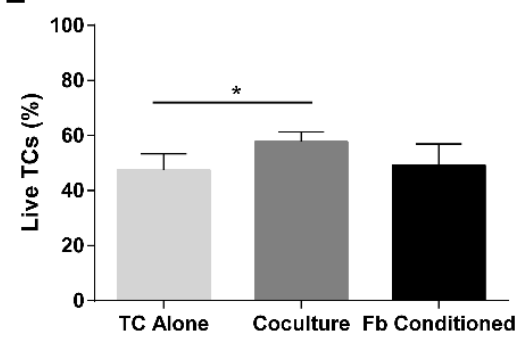

F

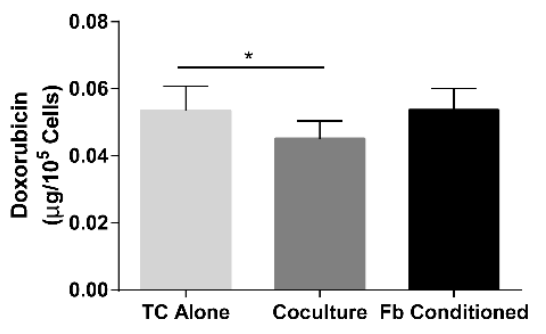

Figure 12| Doxorubicin is less cytotoxic to breast cancer cells cultured in fibroblast-conditioned media or co-culture.

(A) Schematic of conditioned media experiments: Conditioned media is harvested from incubated HDFs (Fbs) after 24 hours and applied to MDA-MB-231 breast tumor cells (TCs). (B) Live TCs assessed by nuclear dead stain $+/$ - doxorubicin $(10 \mu \mathrm{M})$ in $\mathrm{Fb}$-conditioned or control media after 24 hours as percent of total TCs. (n=5) (C) Cellular uptake of doxorubicin by TCs at successive time points after Doxorubicin $(10 \mu \mathrm{M})$ application as assessed by fluorescent signal of lysed cells $(n=6)$. (D) Schematic of insert coculture experiments: MDA-MB-231 cells (TCs) and HDFs (Fbs) are co-cultured independent of contact for 24 hours prior to doxorubicin treatment. In the $\mathrm{Fb}$-conditioned experimental group, the Fbs are removed prior to dosing chemotherapy. (E) Live TCs assessed by nuclear dead stain $+/$ - doxorubicin $(10 \mu \mathrm{M})$ in Fb-conditioned or control media after 24 hours as percent of total TCs. $(\mathrm{n}=5)$ (F) Cellular uptake of doxorubicin by TCs at successive time points after Doxorubicin $(10 \mu \mathrm{M})$ application as assessed by fluorescent signal of lysed cells $(n=6)$. Data are represented as mean \pm SEM. $* p<0.05, * * p<0.01$, $* * * * \mathrm{p}<0.0001$ by paired t-tests $(\mathrm{B}, \mathrm{E}, \mathrm{F})$ and two-way ANOVA followed by post-hoc paired t-tests $(\mathrm{C})$. 
However, there was no difference in doxorubicin accumulation in the conditioned group as compared to the tumor cells alone control. Examination of the fibroblasts also revealed that the co-culture condition not only led to changes in the tumor cell population, but also led to opposite effects in fibroblasts. Co-culture led to increased death of fibroblasts and increased uptake of doxorubicin by fibroblasts (Figure S1). This decrease in doxorubicin accumulation correlated with increased percentage of live cells in comparable conditions after doxorubicin treatment, indicating the potential for fibroblast-mediated alterations in tumor cell drug accumulation and reduced treatment response.

\subsubsection{Cancer cell response to doxorubicin is dependent on the ratio of tumor cells:fibroblasts}

The tumor border includes a transition from the tumor bulk, with relatively few fibroblasts, to the surrounding stroma, with very few tumor cells. Therefore, it was desirable to determine the extent of the fibroblast protective effect within the context of varied cancer cell to fibroblast ratios and varied total cell densities, of relevance to the tumor-stroma transition (TST) zone.

We selected a range of ratios of tumor cells:fibroblasts (TC:Fb) from 4:1 to 1:4 and created 2D cocultures in single wells prior to introduction of doxorubicin (Figure 13A). The total cell number was held constant, and ratios of cells were varied internally. For control comparison, tumor cells were seeded at the same number as the coculture condition in the absence of fibroblasts. No difference was observed in the viability of the single cultured tumor cells with regards to the seeding number (Figure 13B). Contrastingly, the percentage of live tumor cells increased linearly with an increase in the seeding number of tumor cells in coculture with fibroblasts (Figure 13B, S2). Cocultures at 1:4 TC:Fb showed comparable cell survival to single culture conditions, while a ratio of $4: 1$ yielded the greatest percent live. The fibroblast protective effect was, therefore, absent at lower ratios of tumor cells to fibroblasts. 
A

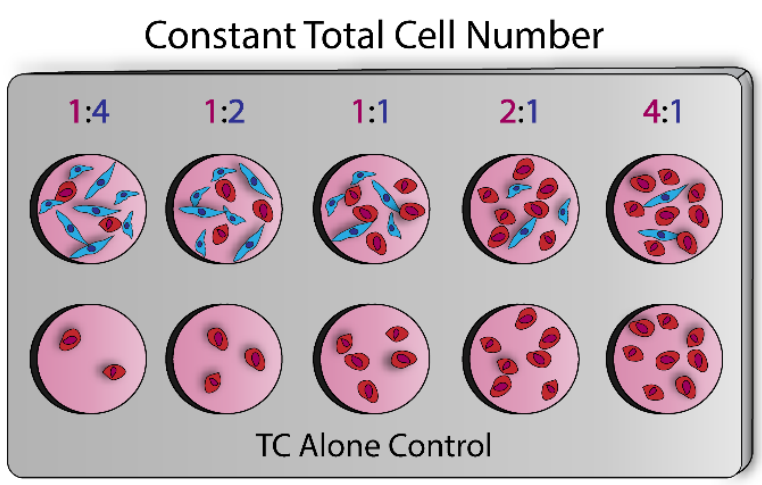

C

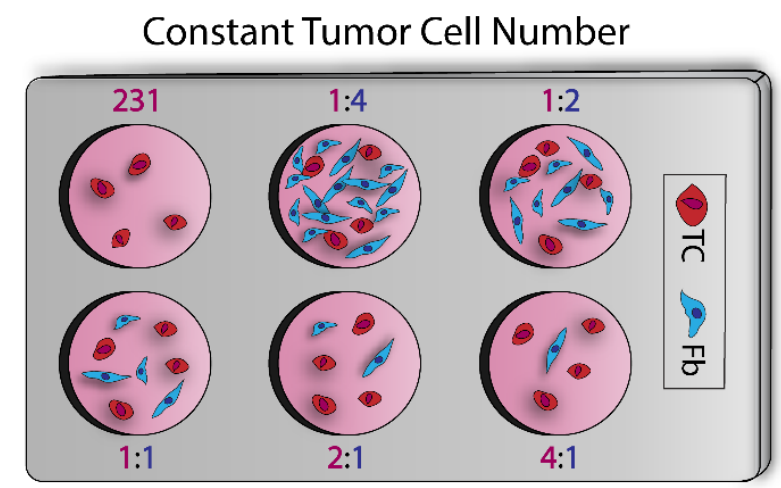

B

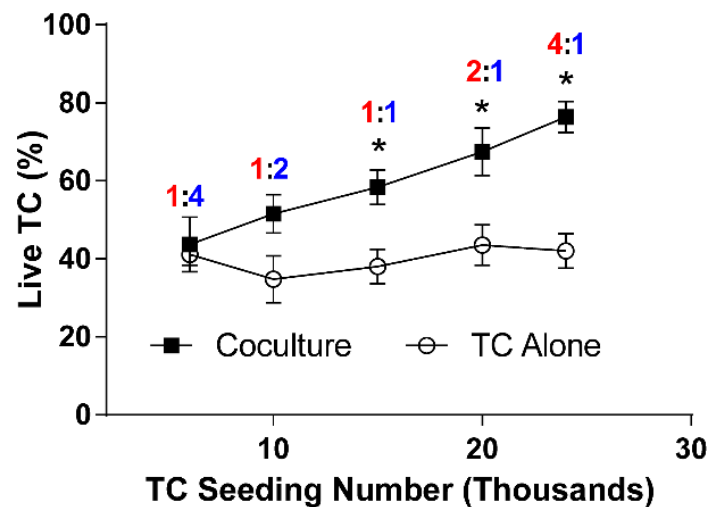

D

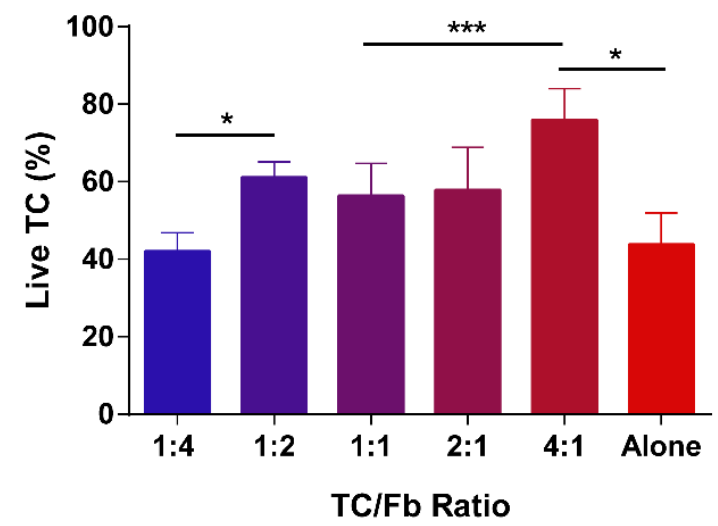

Figure 13| In co-culture, the ratio of tumor cells to fibroblasts alters the viability of tumor cells in response to doxorubicin treatment.

(A) Schematic of constant total cell seeding density experiment: TCs (red) and Fbs (blue) were seeded in a culture dish with the total number of cells (TCs+Fbs) held constant. Single cultured TCs were seeded at the same TC density as experimental conditions with no fibroblasts. (B) Live TCs assessed by nuclear dead stain $+/$ - doxorubicin $(10 \mu \mathrm{M})$ after Doxorubicin treatment for 6 hours with varied ratios of TC:Fb in 2D (n=5) (C) Schematic of constant total cancer cell seeding density: Cells were seeded in a culture dish with the total number of cancer cells held constant. Fibroblast cell numbers were adjusted relative to the cancer cells. Fibroblasts are represented in blue, cancer cells in red. (D) Live TCs assessed by nuclear dead stain $+/$ - doxorubicin $(10 \mu \mathrm{M})$ after doxorubicin application for 6 hours with varied ratios of $\mathrm{TC}: \mathrm{Fb}$ in $2 \mathrm{D}(\mathrm{n}=3)$. Data are represented as mean \pm SEM. $* \mathrm{p}<0.05$, $* * * \mathrm{p}<0.0001$ by post-hoc unpaired t-tests following two-way ANOVA.

To further probe this phenomenon, the number of tumor cells was held constant, and the cell ratio was altered by adjusting only the total number of fibroblasts in coculture (Figure 13C), prior to doxorubicin treatment. A control group was also made by seeding tumor cells at the same number as all other experimental conditions without the addition of fibroblasts. A comparable viability effect was observed as 
seen in the hanging well cultures: A ratio of 4:1 tumor cells to fibroblasts yielded the greatest viability of tumor cells and the greatest difference in viability as compared to the control condition (Figure 13D). These data indicate that the fibroblast protective effect is not dependent on the total number of tumor cells; rather, it depends on the ratio of tumor cells to fibroblasts. Additionally, while fibroblast-derived factors need to be present to observe the effect, the ratio of tumor cells to fibroblasts and not the total number of fibroblasts is the determining factor.

\subsubsection{Ratiometric effects are conserved when cells are cultured in a breast-mimetic 3D microenvironment}

Since the in vivo microenvironment is a 3-Dimensional space with which the cells can readily interact, and it has been shown that dimensionality can affect therapeutic outcomes in cancer, comparable in vitro hydrogel doxorubicin treatment experiments were developed to extend the coculture experimental findings. Collagen-basement membrane hydrogels were developed with varying ratios of tumor cells and fibroblasts found to be relevant $(4: 1,1: 1,1: 4)$ in 2D (Figure 14A,B). Control gels were seeded with tumor cells, alone, at the same total cell number as the coculture hydrogels (Figure 4B). Media with and without doxorubicin was applied to the gels for 18 hours. Total tumor cell viability in the absence of doxorubicin was approximately $90 \%$ for all conditions (Figure S3, 13C). Analysis of the total tumor cell viability within the gels after treatment indicated that a ratio of 4:1 tumor cells to fibroblasts yielded a significantly higher viability than the comparable single culture condition. 
A
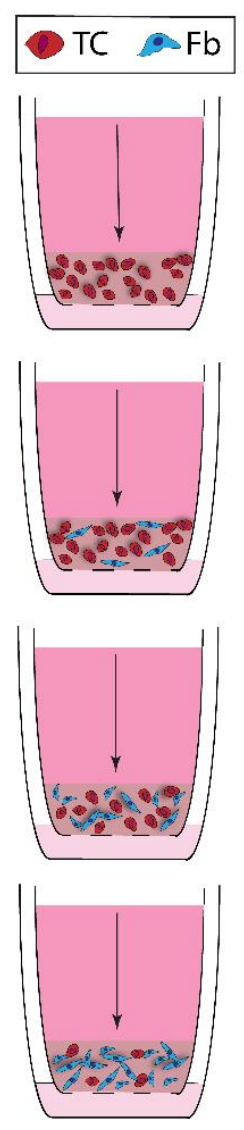

B
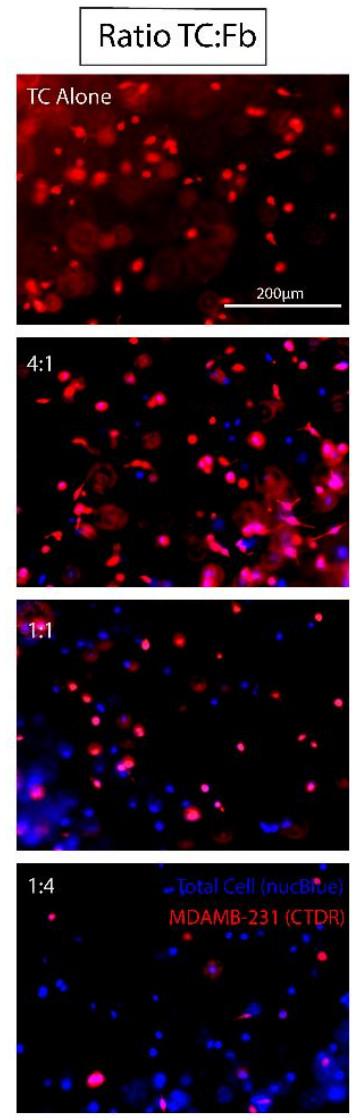

C

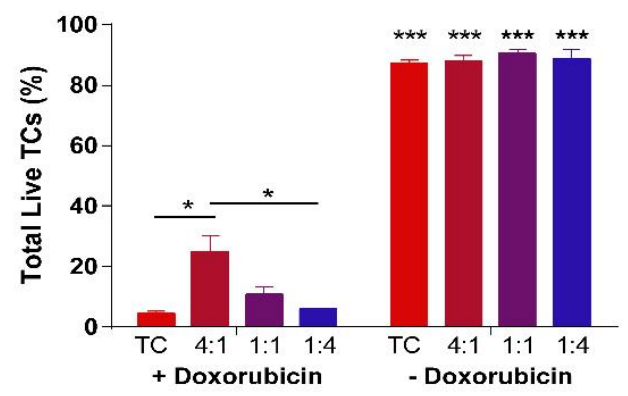

Figure 14| Ratiometric response of tumor cells occurs when cells are co-cultured in a breastmimetic collagen I matrix

(A) Schematic of independent tissue culture insert set-ups for increasing ratios of TCs:Fbs in a collagen I matrix. Arrow indicates direction of application of doxorubicin for 18 hours. (B) Fluorescent images of Celltracker deep red-labeled MDA-MB-231(red, TCs) and total cell nuclei (blue, NucBlue) in 3D collagen hydrogels prior to doxorubicin treatment. Cells were seeded at varied ratios with overall cellular concentration held constant (1E6 cells/mL). Blue label without red indicates a fibroblast. (C) Live TCs assessed by nuclear dead stain within collagen gels $+/$ - doxorubicin $(10 \mu \mathrm{M})$ after $24 \mathrm{~h}$ application via interstitial flow $(n=3)$. Data are represented as mean \pm SEM. $* p<0.01$, $* * * p<0.001$ by post-hoc unpaired t-tests following two-way ANOVA analysis. $* * *$ Signifies statistical significance to matched +doxorubicin condition. 


\subsubsection{Gradients of chemotherapy form across invading edges of tumors}

In addition to the cellular heterogeneity at the tumor edge, increased fluid pressure at the tumor border drives interstitial fluid flow into the adjacent stroma generating a gradient of chemotherapeutic ${ }^{37}$. Collagen hydrogels have been utilized in the past to mimic this region. Here, we developed in silico theoretical concentration profiles within collagen hydrogel mimetics based on the geometry of the hydrogels, Darcy's law of fluid transport, and the general diffusion equation (Table 1). Because the fluid velocity is very low $(\sim 0.5 \mathrm{um} / \mathrm{s})$, concentration gradients develop between the top and the bottom of the gel (Figure 15A). Notably, the concentration of the drug was greatest at the top of the gel relative to the bottom. However, it should be noted that the concentration became homogenous in the hydrogel after 3 hours of simulated flow.

\subsubsection{High tumor cells to fibroblasts ratios reduce cancer cell response in an Agent-Based Model of the tumor microenvironment}

An agent-based in silico model (ABM) was developed using the concentration profiles determined in COMSOL, to represent flow mediated doxorubicin treatment through homogenously seeded hydrogels at varied cancer cell to fibroblast ratios (Figure 15A/B). Predictive equations for the percentage of live tumor cells were determined from the $2 \mathrm{D}$ results (Table 2, S4). Simulations of 18-hour doxorubicin treatment delivered via top-to-bottom interstitial flow indicated an increase in overall percentage of live tumor cells in coculture conditions relative to the tumor cells alone condition (Figure 15C) and this percentage was greater with increasing ratios of tumor cells to fibroblasts.

The ABM was further utilized to probe spatial variations in cancer cell survival through homogenously seeded hydrogels with different tumor cell:fibroblast ratios. At each depth in the gel, greater ratios of tumor cells to fibroblasts yielded a greater percentage of live tumor cells relative to successively lower ratios (Figure 15D). All conditions yielded significantly greater viability than the tumor cell alone control condition. Interestingly, for samples with higher ratios, tumor cells at the top of the gel had a significantly lower percent survival as compared to the bottom of the gel due to drug concentration profiles. 
Our ABM predicted more significant results than our 3D hydrogel experiments, however, the viability trend was comparable between in vitro and in silico models. Viability of tumor cells in each cocultured condition was approximately half of the ABM predicted viability percentage. This is likely due to a reduction in the fibroblast protective effect within $3 \mathrm{D}$ systems, as compared to $2 \mathrm{D}$ culture system, as a result of the extracellular matrix components and greater dispersal of cells.
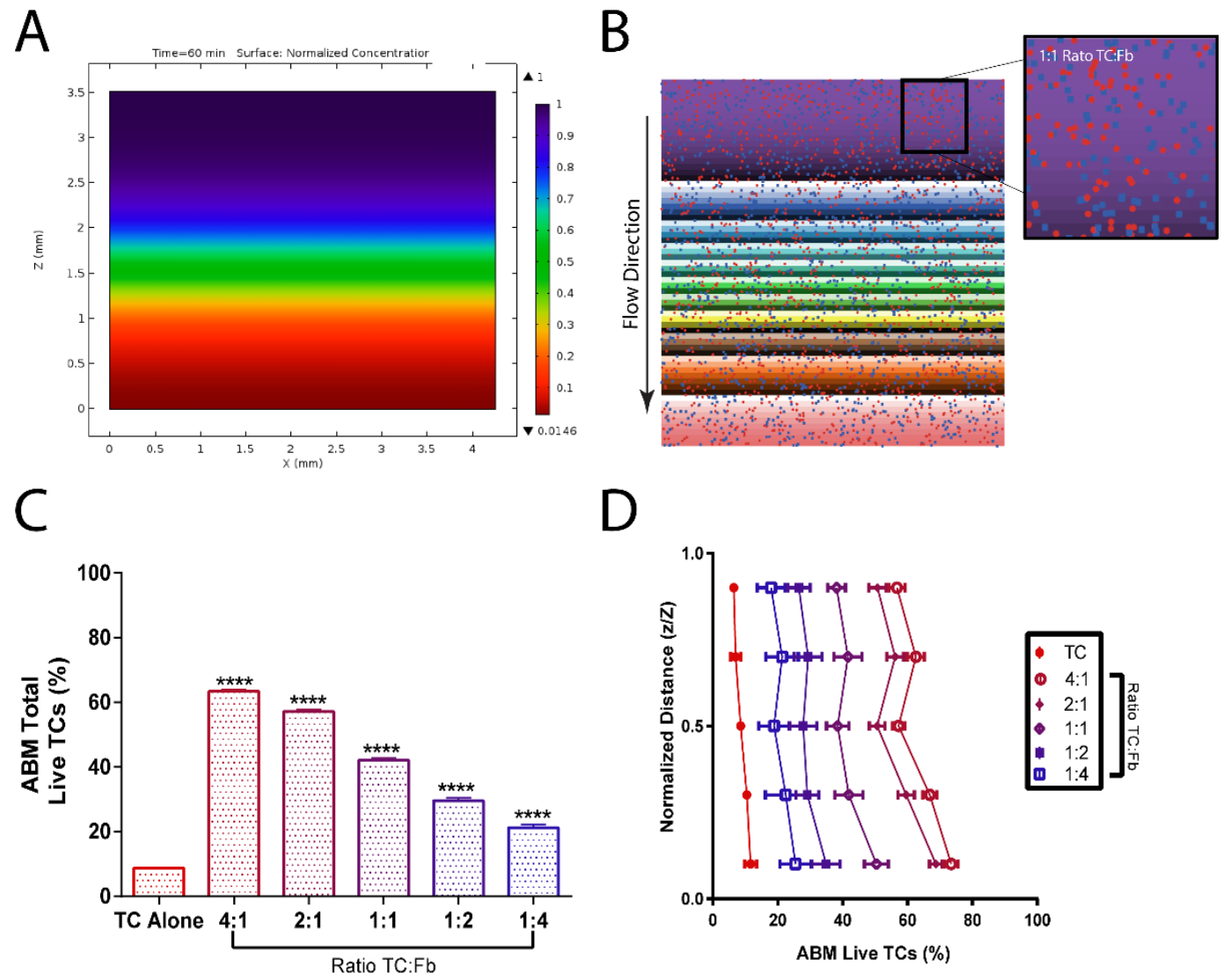

Figure 15| Agent-based model (ABM) predicts overall and distance-dependent response of tumor cells to doxorubicin in the 3D collagen hydrogel system

(A) Diffusion-convection concentration gradient developed in Comsol within a simulated 3D hydrogel. Scale gives normalized concentration $\left(\mathrm{C} / \mathrm{C}_{\max }\right)(\mathrm{B})$ The agent-based model incorporates concentration profiles, cancer cells, and fibroblasts for in silico drug screening. (C) ABM-predicted overall viability for homogenous gels of varied tumor cell:fibroblast ratios $(n=20)(D)$ Location dependent viability of tumor cells in homogenous gels seeded at varied tumor cell:fibroblast ratios $(n=20)$. Data are represented as mean \pm SEM. $* * * * p<0.0001$ compared to 231 control by post-hoc unpaired t-tests following two-way ANOVA analysis. (D) post-hoc t-tests after MANOVA analysis which shows significant effects $(\mathrm{p}<0.001)$ for ratios and distance. 
the invasive edge of tumors

Following confirmation of the fibroblast protective effect in 3D, an in silico ABM tumor to stroma transition (TST) model (Figure 11), representing the in vivo tumor bulk to stroma transition area (Figure 16A), was utilized to predict the region specific effects of doxorubicin treatment within a heterogeneous 3D-hydrogel breast mimetic system. For tumor cell alone simulations, the tumor cells in each successive layer within the simulated hydrogel correspond to the number of tumor cells in the comparable layer in the coculture simulation (Figure 10). 

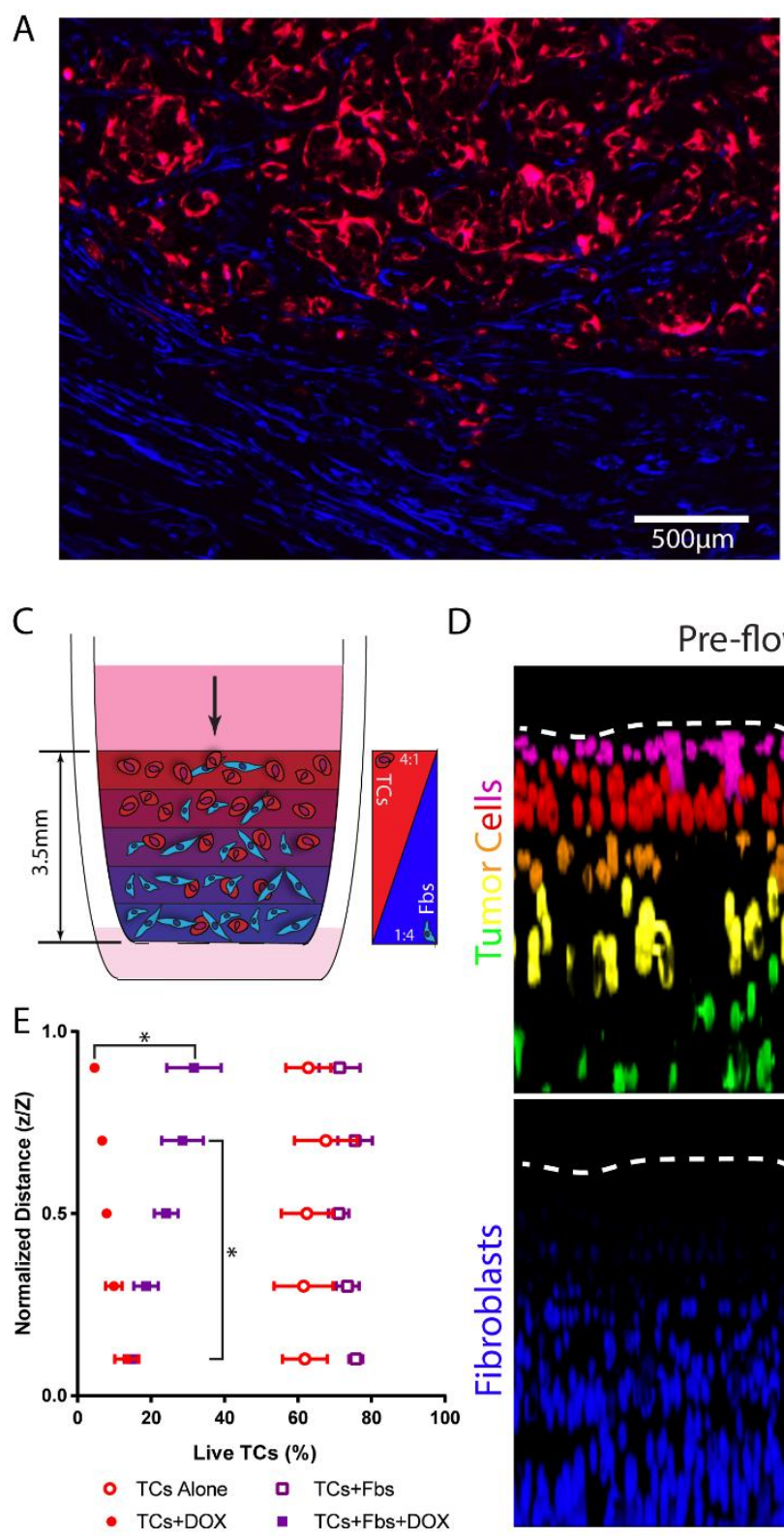

$\mathrm{D}$
B
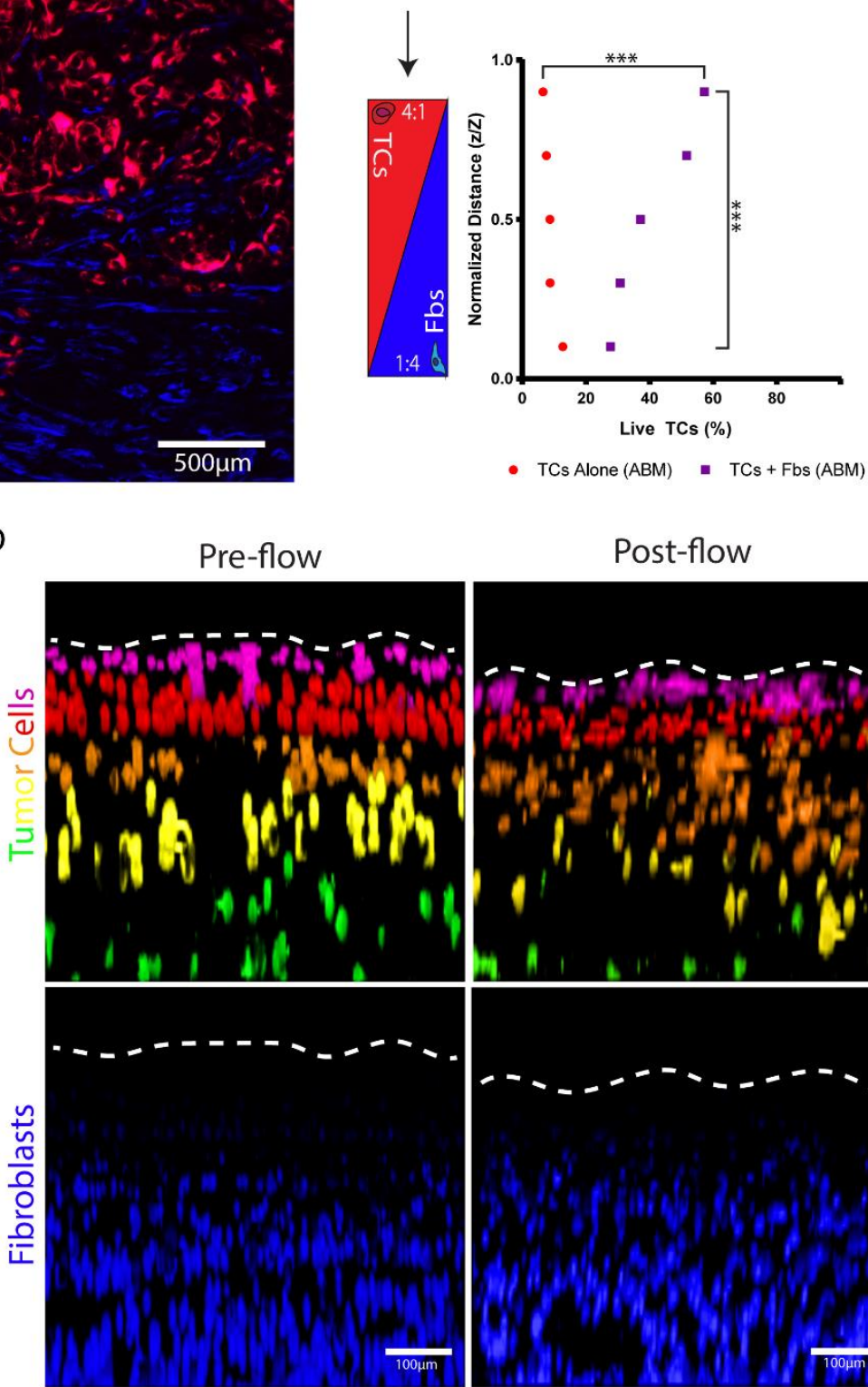

Post-flow
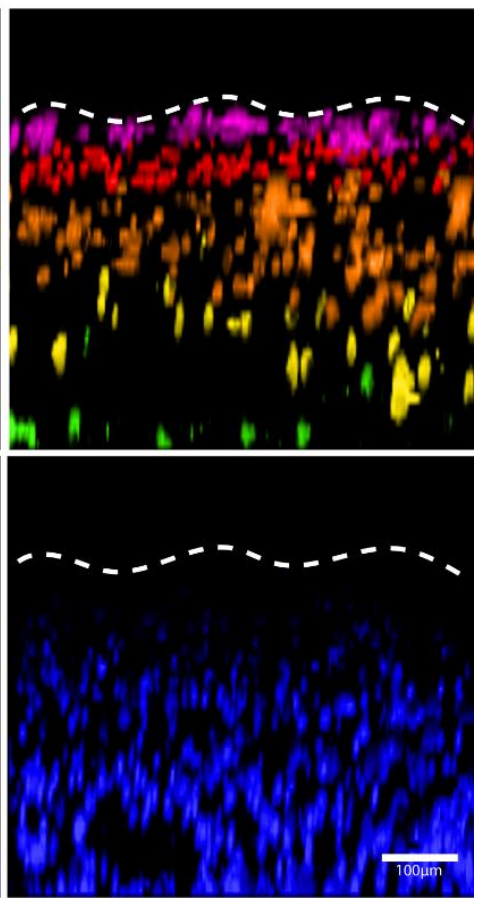

Figure 16 Layered in silico and in vitro models of the tumor to stroma transition zone indicate that tumor cells in the bulk are more viable after doxorubicin than those in the stroma.

(A) Tumor-stroma interface from a resected patient breast carcinoma showing tumor cells (pancytokeratins, red) and fibroblasts (alpha-smooth muscle actin, blue). (B) ABM predicted percent viability in varied depths within a layered hydrogel with counter-correlated gradients of TCs and Fbs after 18 hours of simulated drug treatment. $(n=20)$. (C) Schematic of a penta-layered collagen hydrogel setup in a tissue culture insert with countercorrelated gradients of TCs and Fbs to model the tumor to stroma transition zone (TST model). (D) Confocal images of the TST model under control (no treatment) condition prior to (left) and after (right flow) with tumor cells (false colored -top) and fibroblasts (bottom). The tumor cells are labelled with alternating cell tracker dyes (green and deep red) to distinguish each layer. Dotted line indicates top of gel. (F) Live TCs assessed by nuclear dead stain within collagen gels $+/$ - doxorubicin $(10 \mu \mathrm{M})$ and $+/$ - fibroblasts at varied depths within the hydrogel for 18 hours of treatment. $(\mathrm{n}=3)$ Data are represented as mean \pm SEM. $* \mathrm{p}<0.05, * * * \mathrm{p}<0.001$ by t-test after MANOVA analysis showing significance for distance and group. 
Results predicted that tumor cells alone, region specific, cell viability followed the same trend as homogenously seeded tumor cell alone simulations (Figure 16B), where cancer cell populations that encountered a higher concentration of drug for the longest period of time (top of the gel) had significantly lower viability compared to cell populations at the bottom of the hydrogel, that encountered a lower average concentration of drug. Interestingly, this trend was reversed for coculture simulations. Tumor cells at the top of the hydrogel, representative of the tumor bulk region, had a greater percentage of live tumor cells as compared to the cancer cell populations at the bottom of the gel, representative of the tumor stromal region. The simulations indicated that tumor cells in the tumor bulk are protected from doxorubicin chemotherapy to a greater extent than tumor cells in the simulated stroma, indicating the potential for a resistant subpopulation within the tumor microenvironment.

\subsubsection{A corresponding in vitro model of the invasive edge indicates similar region specific cancer cell} response

A penta-layered hydrogel in vitro TST model was created by successively depositing hydrogel solutions with increasing cancer cell to fibroblast ratios from bottom to top of a cell culture insert (Figure 16C). Tumor cells in alternating layers were labelled with different cell tracker dyes (Figure 16D). Distinct regions were confirmed, via confocal imaging, prior to introduction of flow. These regions were confirmed following flow application (Figure 16D).

The region-specific viability within the in vitro TST model confirmed the trends predicted by the comparable ABM (Figure 16E), with the addition of fibroblasts increasing viability overall. However, fibroblasts did not confer significantly increased viability in the lowest layer of the TST model as was predicted by the ABM. Tumor cells alone with flow-applied doxorubicin treatment yielded regions at the top of the gel with significantly decreased percentages of live tumor cells as compared to the bottom the hydrogel. By contrast, coculture (TCs+Fbs) hydrogels yielded significantly greater cancer cell viability at 
the top of the gel as compared to the bottom of the gel. In the absence of doxorubicin, there was no difference in region specific viability for cocultured and tumor cell alone TST models.

\subsubsection{The interaction of transport properties with cellular interactions in the ABM shows the importance}

of tissue- level changes in cancer cell response to chemotherapy

Sensitivity analyses of COMSOL parameters emphasize the effects of transport properties on region specific and average cancer cell viability (Figure 15A). Diffusion alone, in the absence of convection, was not a major mediator of cancer cell death, as drug does not penetrate into the gel after 18 hours (Figure 16). Transport from the bottom of the gel to the top, simulating flow from the stroma to the tumor, yielded an even greater difference in the viability of the cells at the top relative to the bottom of the gel, which yielded a corresponding overall increase in cancer cell viability as compared to the normal flow control (Figure 15B). 

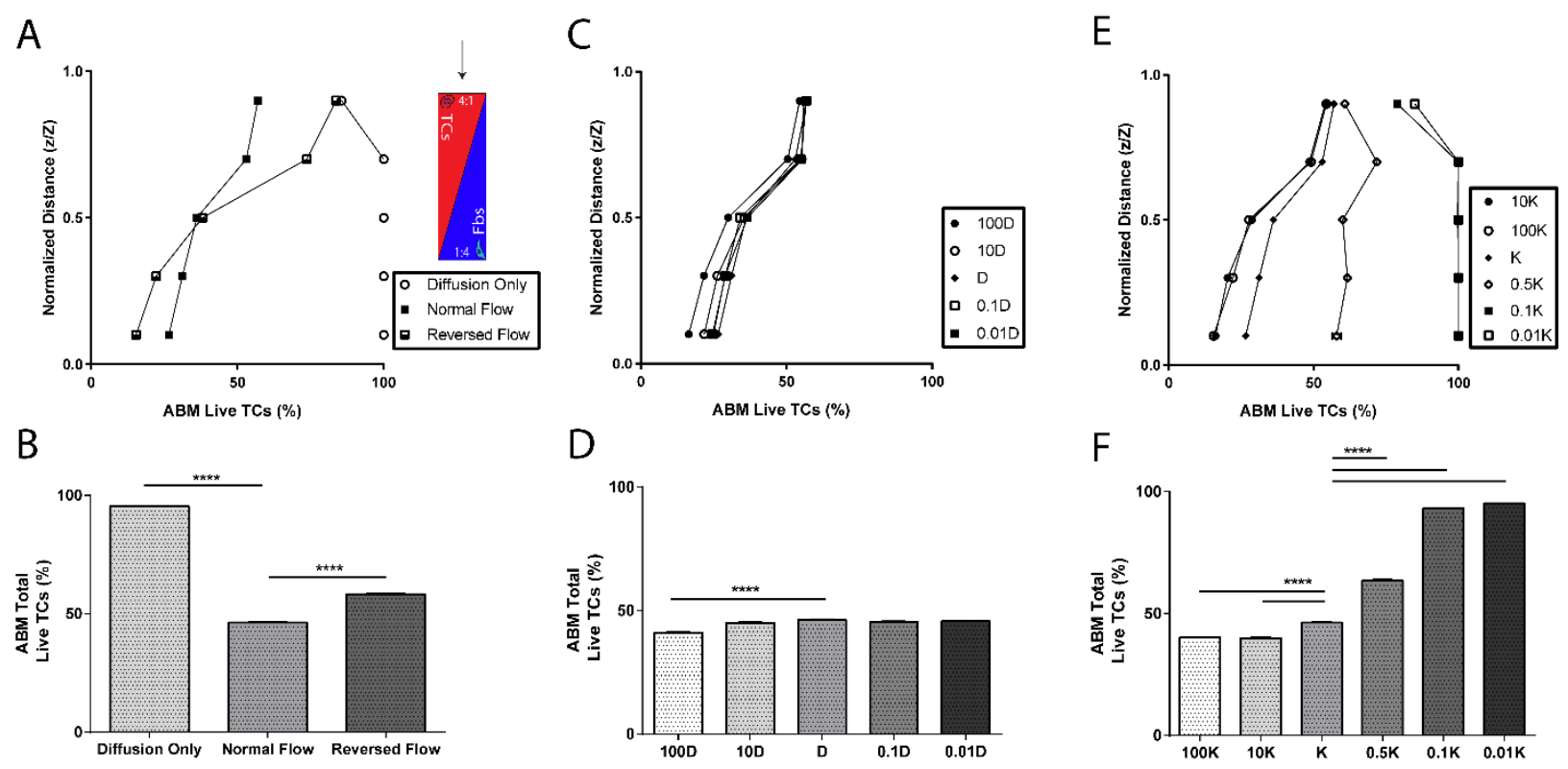

Figure 17| The agent-based model reveals complex interactions between transport properties and cellular response to chemotherapy across the TST Zone.

Cancer cell viability at increasing depth through the agent-based TST model for varied (A) transport conditions including normal flow (top to bottom), reversed flow (bottom to top) and no flow (diffusion only) (C) diffusion coefficients, and (E) permeability coefficients, after 18 hours of simulated treatment with $10 \mu \mathrm{M}$ doxorubicin. $(\mathrm{n}=20)$ Total cancer cell viability within the agent-based TST model for varied (B) transport conditions, (D) diffusion coefficients, and (F) permeability coefficients, after 18 hours of simulated treatment with $10 \mu \mathrm{M}$ doxorubicin. D indicates baseline diffusion coefficient value $\left(6 \mathrm{e}^{-11}\right.$ $\left.\left[\mathrm{m}^{\wedge} 2 / \mathrm{s}\right]\right)$, and $\mathrm{K}$ represents baseline permeability coefficient value $\left(3.19 \mathrm{e}^{-14}\left[\mathrm{~m}^{\wedge} 2\right]\right)(\mathrm{n}=20)$. Data are represented as mean \pm SEM. $* * * * \mathrm{p}<0.0001$ by post-hoc unpaired t-test following MANOVA (A,C,E) and by post-hoc unpaired t-tests following two-way ANOVA analysis (B,D,F). All main effects were significant by MANOVA or ANOVA.

Altering the diffusion coefficient, analogous to changing the size of the drug, while maintaining convective parameters, yielded no significant difference in the general region specific viability of tumor cells (Figure 17C), except for a 100-fold increase in the diffusion coefficient. Similarly, overall percent live tumor cells within the hydrogels was only significantly altered from the control condition in the case of 100-fold increase in the diffusion coefficient (Figure 17D). Analysis of the concentration profiles indicated that convection was dominating diffusion in this context (Figure 18). 

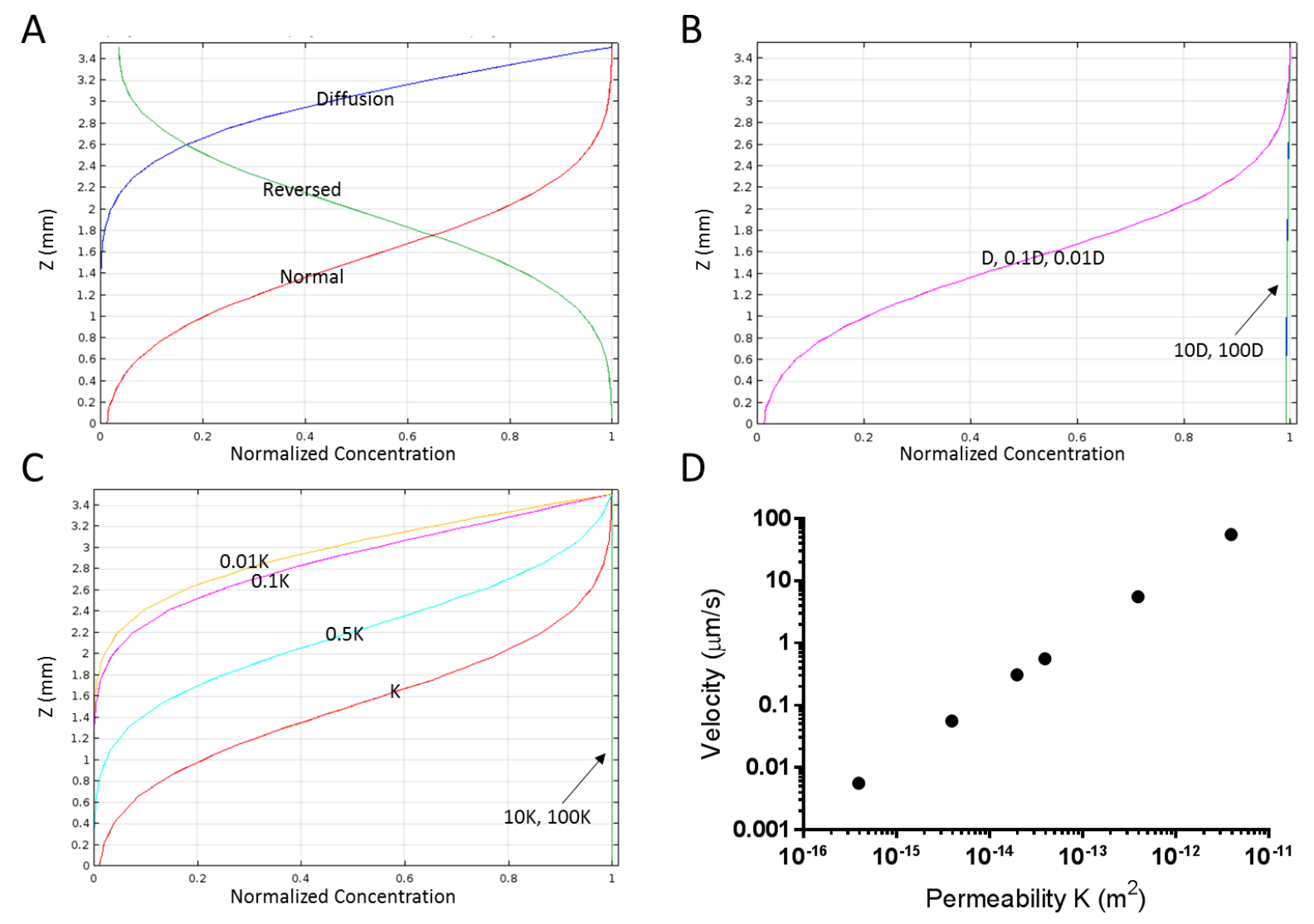

Figure 18| The agent-based model reveals complex interactions between transport properties and concentration profiles.

Normalized drug concentration within a simulated cancer cell and fibroblast coculture layered gel at varied depths for varied (A) types of transport, (B) diffusion coefficients, and (C) permeability coefficients. Data is represented at 60 minutes. D indicates baseline diffusion coefficient value $\left(6 \mathrm{e}^{-11}\right.$ $\left.\left[\mathrm{m}^{\wedge} 2 / \mathrm{s}\right]\right)$, and K represents baseline permeability coefficient value (3.19 $\left.\mathrm{e}^{-14}\left[\mathrm{~m}^{\wedge} 2\right]\right)$. (D) Simulated fold changes in permeability relate to comparable fold changes in fluid velocity through the simulated hydrogel. The arrow indicates the baseline permeability $\left(3.19 \mathrm{e}^{-14}\left[\mathrm{~m}^{\wedge} 2\right]\right)$

Alterations of the permeability coefficient, analogous to altering the matrix density, and associated alterations in velocity (Figure 18D), while maintaining the diffusion parameters, yielded varied alterations in region specific and overall cancer cell viability (Figure 17E). While the viability at the top of the gels was always greater than the bottom of the gel, for comparable conditions, increasing the permeability decreased the overall viability (Figure $17 \mathrm{~F})$. At low values of permeability $(0.01 \mathrm{~K}$ and $0.1 \mathrm{~K})$ there was no difference in region specific or overall viability, however, the viability was not zero. This was due to the fact that the gel becomes saturated with drug almost instantly at these values; therefore, the viability effects 
become dominated by the drug dosage and the fibroblast protective effect. At very low values of permeability, the region specific and overall viability was comparable to diffusion only conditions, indicating that diffusion was dominating convection in these conditions.

\subsection{Discussion and Conclusion}

\subsubsection{Mechanisms behind fibroblast induced chemotherapy resistance}

Multidrug resistance in cancers is a documented phenomenon, and can be meditated by several cellular mechanisms; however, anthracycline resistance is dominated primarily by classical multidrug resistance ${ }^{40}$. Classical multidrug resistance is described by acquired resistance to chemotherapy by lowered intracellular drug concentration; this can be mediated by increased drug efflux or decreased drug uptake. For anthracyclines, such as doxorubicin, intracellular drug accumulation is decreased by increased drug efflux via ATP-dependent transporters ${ }^{96}$. Shen and colleagues ${ }^{97}$ describe the uptake and efflux of doxorubicin within wildtype and multidrug resistant MDA-MB-435 cells and indicate that multidrug resistance in breast cancer cell lines is dominated by increased drug efflux. Therefore, it is predicted that the decrease in cancer cell doxorubicin concentration and comparable increase in the percentage of live tumor cells may be due to classical drug resistance, mediated by drug efflux. Additionally, fibroblast doxorubicin accumulation in the coculture condition was not negligible, however, the concentration of extracellular doxorubicin after 6 hours $(\sim 8 \mu \mathrm{M})$ was still above the determined IC50 (3.7 $\mu \mathrm{M})$ for MDAMB-231 cells. Two studies have found that both the cytoplasm and the nucleus of MDA breast tumor cells become saturated prior to 6 hours at varied doses of doxorubicin ${ }^{97,98}$, and this saturation occurs even at a dosed doxorubicin concentration as low as $5 \mu \mathrm{M}^{97}$. Therefore, it is not likely that the reduced viability in coculture conditions is simply due to uptake by stromal fibroblasts. That being said, localized effects of this accumulation could have further reaching effects on total tumor treatment response based on observations of gradient formation of doxorubicin in patients and resistance to treatment ${ }^{99}$.

One of our more perplexing findings is that there is a protective effect at greater ratios of tumor cells to fibroblasts, yet mechanisms underlying this response are more challenging to parse. From our data, 
we interpret that a concentration-dependent, fibroblast-derived signaling molecule is necessary for the protective effect to occur, and this effect is observed at higher ratios of tumor cells to fibroblasts, regardless of the overall cell numbers. In other words, increasing the number of fibroblasts relative to tumor cells decreases the protective effect. Fibroblast activation, a major hallmark of cancer-associated fibroblasts, has been attributed to TGF $\beta$, reactive oxygen species, and modifications to the extracellular matrix, among other mechanisms. Activation of fibroblasts leads to further secretion of TGF $\beta$, as well as increased secretion of other cytokines such as IL-6 and extracellular matrix molecules such as Tenascin $\mathrm{C}^{100}$. TGF $\beta$ and IL-6 both increase resistance to anthracycline chemotherapy in breast cancer ${ }^{101,102}$, and Tenascin C is associated with chemoresistance in lung cancer ${ }^{103}$. It's possible that in our system, the ratiometric effect is due to a unique balance between signaling molecules from cancer cells to fibroblasts on a per cell basis needing to be higher than signaling molecules from fibroblasts to cancer cells, but the effect is amplified regardless when cells are touching or sharing an extracellular matrix as in our co-culture systems. Fibroblasts have also been associated with creating niches in which cancer stem cells, a particularly drug resistant population of cancer cells, reside and proliferate ${ }^{104}$. The ratio effect may be due to finding a particular sweet spot for creation of such a niche for these chemoresistant cells.

Regardless, the fibroblast protective effect is a dominating factor for increased cancer cell viability in our 2D and 3D in vitro systems. In silico homogenous gel models predict that region specific cancer cell viability is affected by chemotherapy gradients; however, our in vitro and in silico models predict that the overall viability is dominated by the dependent fibroblast protective effect. This indicates that viability trends within homogenous tumor regions are predicted by concentration gradients, yet the magnitude of the viability percentage may be dominated by the relative abundance of fibroblasts.

However, within regions of cellular heterogeneity, the region-specific viability response to doxorubicin treatment is not solely predicted by the average doxorubicin concentration that the tumor cells experience (Figure 5). While tumor cells at the top of the hydrogels, representative of the tumor bulk region, experience a greater average concentration of drug as compared to the bottom of the gel, representative of 
normal stroma, the viability in this region is greater. This indicates a potentially important subpopulation for cancer progression and recurrence.

\subsubsection{Drug transport in the tumor microenvironment}

Fluid dynamics can alter drug concentration gradients and affect region specific and overall viability trends in the tumor border region ${ }^{95,34}$. Reversed flow enhances the cancer cell viability in the bulk region and simultaneously decreases the viability in the stromal region, while additionally increasing the overall viability. This condition is of import, as the direction of fluid velocity in vivo is not known, and regional tumor heterogeneity could yield solute influx into the tumor from the surrounding stroma ${ }^{34}$. Diffusion, in the absence of convection, does not alter viability at 18 hours of simulation due to lack of solute penetration into the hydrogel. These results indicate that convection is a dominating factor for concentration profile development and corresponding cancer viability response ${ }^{37}$. This is further confirmed by alterations of the diffusion coefficient, while simultaneously keeping convection constant. In these simulations, the diffusion coefficient only affects the viability at very large values. This confirms that convection is the driving force for doxorubicin concentration development, and diffusion dominates only when molecules are very small.

These results are comparable to those reported by Jain and colleagues ${ }^{29,30,34,35}$; the authors highlight the importance of convection and diffusion for solute transport in neoplastic tissues. Specifically, convection is dominant in these tissues when the molecular weight of the solute is significantly large, while diffusion dominates with very small particles ${ }^{32}$. This rationale was further used to explore the transport effects of large molecules within the tumor interstitium and corresponding implications for antibody-based drug delivery ${ }^{30}$. The results indicate that high molecular weight drug efficacy, in whole tumors, is attenuated by extravasation from the tumor bulk. While doxorubicin would not be considered a large molecule drug, it is well within the size range for convection driven flow in neoplastic tissues ${ }^{34}$.

However, convection dominance is also mediated by the permeability of the tissue of interest. As breast tumor heterogeneity affects the permeability and corresponding fluid velocity ${ }^{105}$ in vivo, 
characterizations of alterations in permeability were explored to determine the effect of tumor permeability heterogeneity on cancer cell viability. Physiologic breast tumor interstitial fluid velocities are predicted to be between $0-10 \mu \mathrm{m} / \mathrm{s}$ as compared to normal interstitial velocities between $0.1-1 \mu \mathrm{m} / \mathrm{s}^{53}$. The permeability sensitivity analysis accounted for velocity alterations within these ranges. Diffusion dominates convection when interstitial velocities are decreased to normal tissue values $(0.1 \mathrm{~K}$ and $0.01 \mathrm{~K})$, as is apparent from comparison to the diffusion alone concentration profile. Similarly, regional viability was comparable between the low permeability and diffusion only conditions. As permeability is increased, viability decreases due to rapid homogenization of concentration gradients. Therefore, decreasing the permeability would decrease the extravasation rate from the tumor bulk and subsequently increase the efficacy of overall drug penetration into the tumor, previously reported as a method to increase whole tumor therapeutic efficacy $^{106}$. However, regional drug efficacy at the tumor border could be potentially attenuated with decreased permeability, as dispersed concentration profiles would develop in this region.

\subsubsection{Implications of our models to therapeutic efficacy and clinical response}

Together our data indicate that several TME-specific factors affect cancer cell viability within the tumor stoma transition region. Interestingly, we see that it is the cells nearest the modeled invading stroma that are more susceptible to therapy than those in the tumor bulk. There is a great deal of research focused on targeting or preventing development of invading cells within these stromal zones ${ }^{55,107-109}$ as a means for preventing metastasis. Examination of tumor cells live in vivo indicates heterogeneity in the subsets of cells that invade through tissues ${ }^{110}$. Though it is intuitive to think that invaded cancer cells will not be accessed by therapies and thus will continue to invade and lead to systemic metastases, our data here may indicate that cells closer to the bulk may resist treatment better and potentially become invading cells posttherapeutic administration. Further, our results are limited by only examining one breast cancer cell line, MDA-MB-231 and one fibroblast type. Due to the ubiquitous presence of fibroblasts in carcinoma develop in multiple organs, our findings may translate in part, though broader interpretation requires further investigation to understand the interaction of intercellular tumor-stroma signaling and transport. This is 
particularly true for highly desmoplastic cancers, such as pancreatic carcinoma, which are known to be highly transport limited with extreme ratios of stroma to cancer compared to other solid tumors ${ }^{111,112}$.

\subsubsection{Conclusion}

Here we find that there is a distinctive interaction between the contributions of fibroblasts and transport to the activity of a common chemotherapeutic, doxorubicin, in a simulated breast cancer microenvironment. Our findings indicate that specific ratios of tumor cells to fibroblasts result in increased drug resistance and that these forces are dominant over transport limitations within our particularly in vitro and in silico models. These results indicate that it is important to study transport and cellular interactions simultaneously when examining cancer-related therapy as both factors play an important role in the in vivo response. 


\section{CHAPTER 5: Conclusion}

The current work describes in vitro and in silico models utilized to probe the effects of the TME on cancer progression and metastasis. Together, these models characterize TME-specific effectors of cancer cell response and motivate further explorative research. Two distinct agent-based models were developed to analyze and predict the factors that correlate with cancer development and progression. The first model provides evidence for two major mechanisms of increased invasion in the brain, in the presence of interstitial flow. The second model predicts a specific cellular population that could be resistant to chemotherapy at the tumor border due to unique TME factors.

Additionally, in vitro 2D and 3D models elucidate the effects of fibroblast on cancer cell resistance to doxorubicin therapy. Novel in vitro layered transition hydrogel models were utilized to probe the region specific effects of fibroblast resistance. Due to therapeutic transport factors and the protective effect, regional variations in viability in response to flow-delivered therapy were observed, as predicted by agent-based models. Together these data indicate the importance of TME factors in altering cancer cell behavior, and predictive models further confirm these effects in a replicable manner.

Our in vitro layered model replicates the morphology of the tumor border transition and could be utilized for further probing of the population specific phenomenon observed through interactions with fibroblast. The specific model could be tuned to incorporate varied cell densities, cancer subtypes, and chemotherapy drugs. The layered hydrogel model can further be utilized to probe the effects of transport on therapeutic efficacy by modulating local ECM composition.

Most importantly, characterization of two separate in silico models with distinct cancer types indicates the transferability and utility for agent-based model use in multiple tumor microenvironments. Components of these model can now be utilized to predict cancer response in more complex contexts. While migration was not incorporated into the ABM of the breast cancer TME due to the minimal cancer cell velocity observed with live imaging ${ }^{53}$, research has shown that overall cancer cell migration is increased with fibroblast coculture ${ }^{113}$. This indicates a potential for probing the mechanism of increased 
invasion within breast cancer coculture conditions using the methodologies established for brain cancer migration. Further probing these migration characteristics in the presence of interstitial flow delivered chemotherapy will provide a broader understanding of invasive subpopulations within the breast cancer microenvironment.

Alternatively, little is known about the effects of chemotherapy on the invasive characteristics of glioma cells. Standard of care treatment for glioma patients includes adjuvant temozolomide treatment ${ }^{114}$, therefore, interstitial flow delivered chemotherapy is also of importance in the brain. Incorporating chemotherapy treatment into the established agent-based model of invasion mechanisms could provide insight into resistant subpopulations within the diffuse invasive edge, characteristic of brain cancers following surgical resection. Furthermore, it has been established that tumor associated cells in brain cancer alter the migration characteristics of glioma cells, and the presence of astrocytes within the brain TME limits patient survival in vivo ${ }^{64}$. Thus, it is possible that a similar cancer protective effect could be observed in brain tumors as in breast tumors, and the tumor border transition area could, likewise, be an important niche for protected cancer subpopulations.

Altogether, our research provides support for the pertinence of agent-based models for TME applications. Results obtained from in silico models demonstrate the usefulness of agent-based models for predicting the behavior of cancer cells in the TME, meditated by complex environmental factors. The versatility of this modeling strategy is confirmed through the predictive simulations that represent in vitro findings in two varied TME contexts. Thus, agent-based models are an optimal modeling platform for synthesizing complex interactions into tunable, robust representations of physical processes, and the techniques developed in this work could be utilized to predict similar effects in comparable settings. 


\section{Supplemental Figures}

\section{Appendix}

A

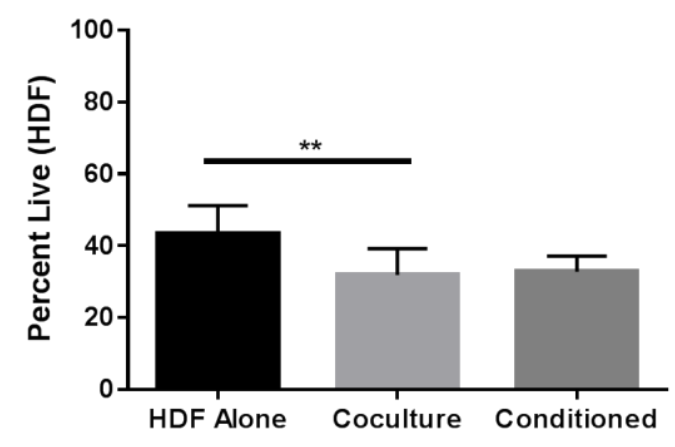

B

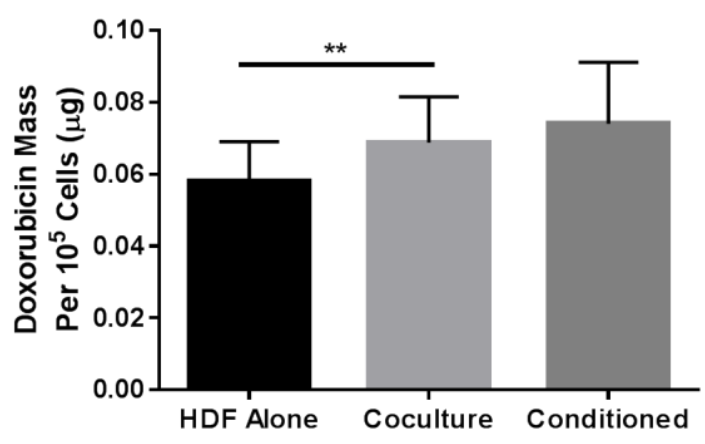

Figure S1| Fibroblast viability is lessened in the presence of cancer cells within hanging-well 2D culture.

MDA-MB-231 cells and HDFs are cocultured independent of contact for 24 hours prior to $10 \mathrm{uM}$ doxorubicin treatment. In the conditioned experimental group, the $231 \mathrm{~s}$ are removed prior to dosing chemotherapy. (A) Percentage of live HDFs after 6 hours of doxorubicin chemotherapy treatment $(n=5)$. (B) Uptake of doxorubicin following 6 hours of treatment, here the total mass of uptake accounts for $\sim 10 \%$ of the total available extracellular doxorubicin $(n=6)$. Data are represented as mean \pm SEM. ** $\mathrm{p}<0.01$, by post-hoc paired t-test following two-way ANOVA. 

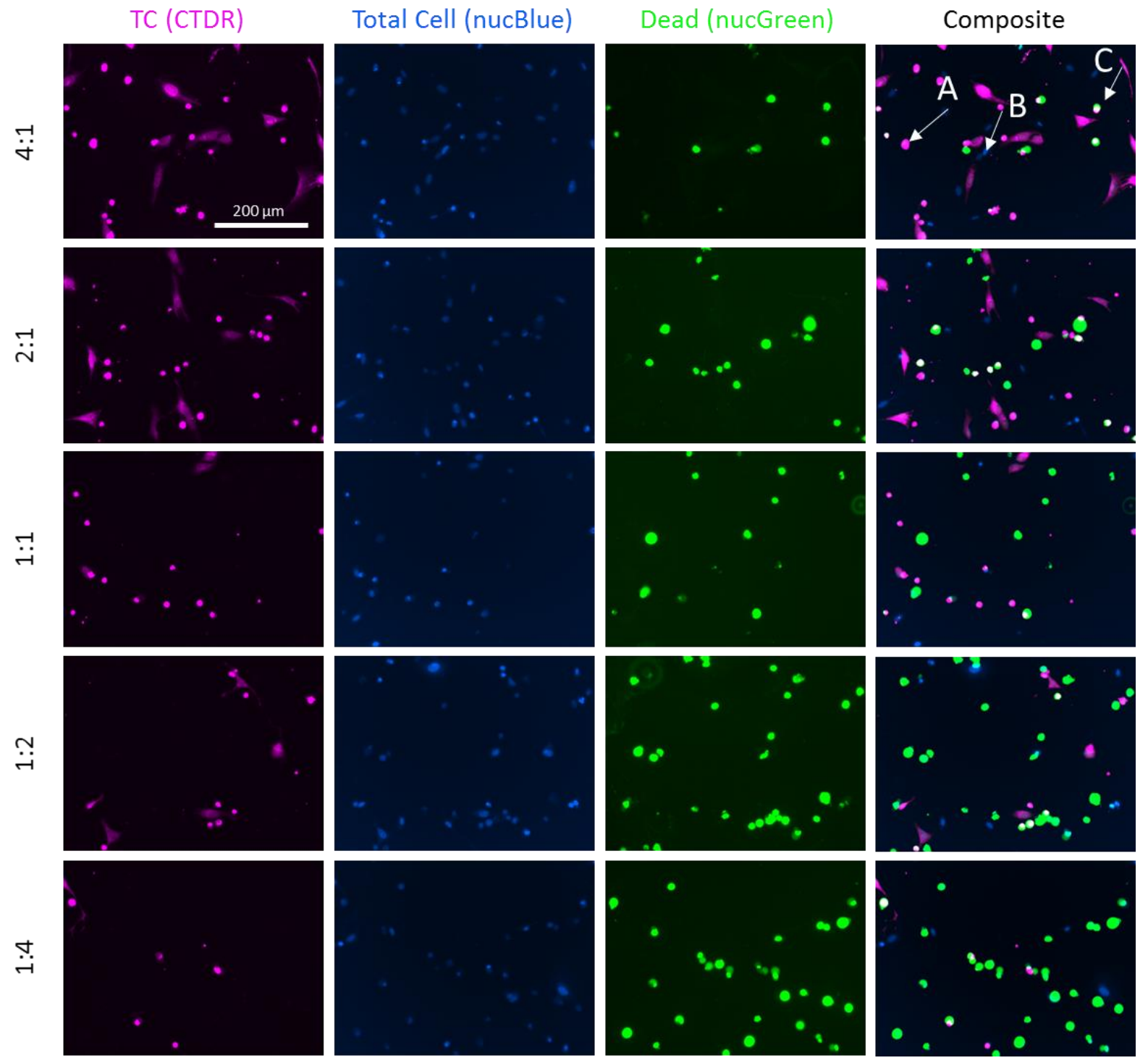

Figure S2| Sample panel for image analysis in 2D coculture experiments.

Sample images for live/dead analysis within 2D coculture systems (See Figure 12a). Here the experimental conditions are portrayed. The first column indicates the total cancer cells stained with deep red cell tracker. The second column indicates the total nuclei of all cells stained with a nucBlue indicator. The third column indicates dead cells labelled with nucGreen. The fourth column shows the composite image. Dead cancer cells were determined by localization of all three channels (C). (B) Indicates a fibroblast cell, and (A) indicates a cancer cell. 

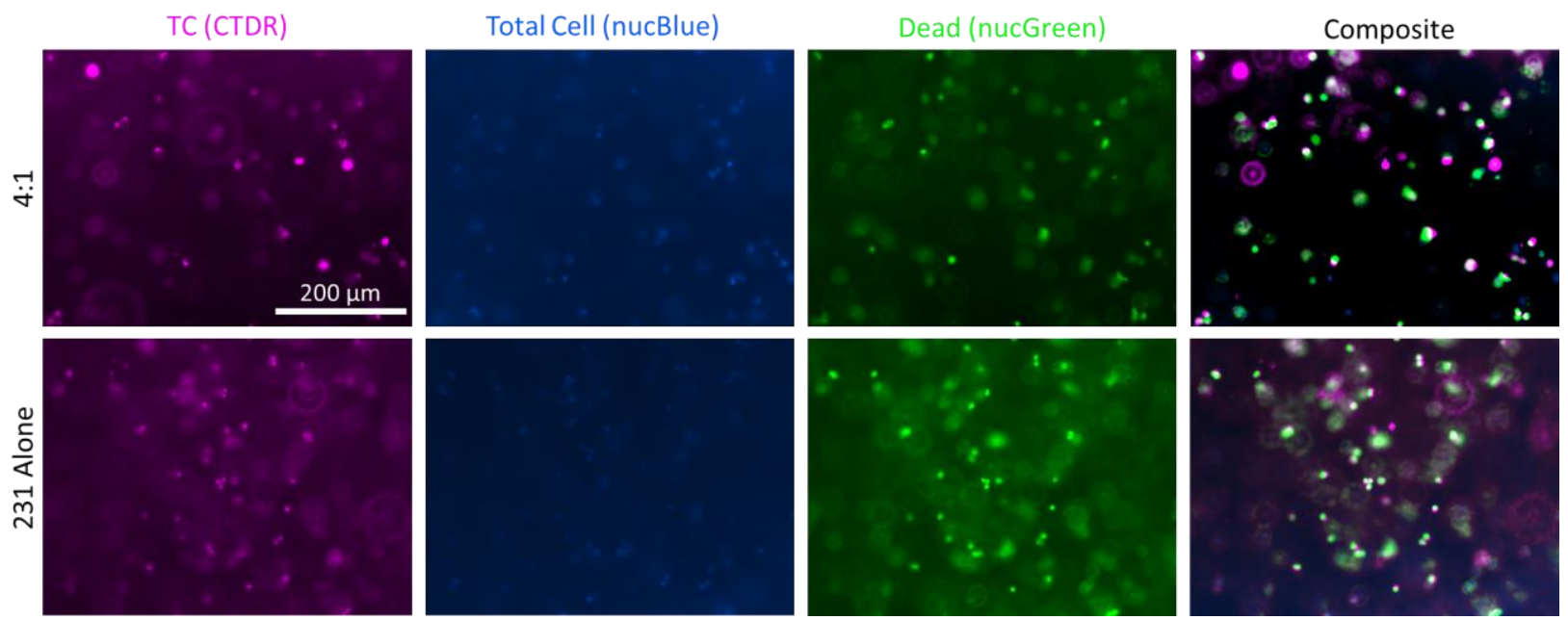

Figure S3| Sample panel for image analysis in 3D coculture experiments.

Sample images for live/dead analysis within 3D homogenous hydrogels. Here the 4:1 and single culture control condition are shown. The first column indicates the total cancer cells stained with deep red cell tracker. The second panel indicates the total nuclei of all cells stained with a nucBlue indicator. The third column indicates dead cells labelled with nucGreen. The fourth column shows the composite image. Dead cancer cells were determined by localization of all three channels.
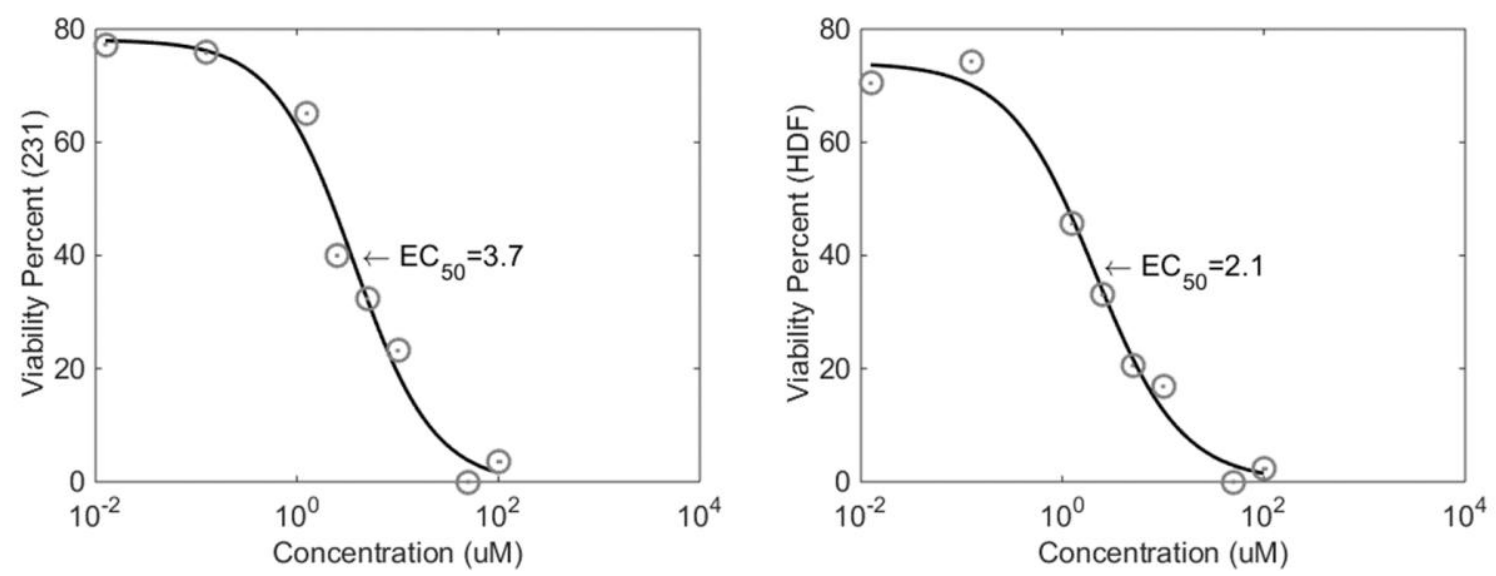

Figure S4| 2D doxorubicin EC50 curves for HDFs and MDA-MB-231 incorporated into the agentbased models.

(A) MDA-MB-231 EC50 curve following 24 hours of treatment at varied concentrations of doxorubicin $(n=3)$. (B) HDF EC50 curve following 24 hours of treatment at varied concentrations of doxorubicin $(n=3)$. Data are represented as the mean of 3 independent trials. Curve fits were determined using an EC50 curve fitting Matlab algorithm. 


\section{Acknowledgements}

The researchers would like to acknowledge Lynette Sequeira for her technical laboratory assistance and Charles Calderwood for statistical assistance. We thank RC Cornelison, RP Pompano, SM PeirceCottler, and SS Blemker for helpful discussion. We also thank the rest of the Munson lab for helpful discussion. 


\section{References}

1. Shieh AC. Biomechanical forces shape the tumor microenvironment. Ann Biomed Eng.

2011;39(5):1379-1389. doi:10.1007/s10439-011-0252-2.

2. Whiteside TL. The tumor microenvironment and its role in promoting tumor growth. Oncogene. 2008;27(45):5904-5912. doi:10.1038/onc.2008.271.

3. Hanahan D, Weinberg RA. The Hallmarks of Cancer. Cell. 2000;100(1):57-70. doi:http://dx.doi.org/10.1016/S0092-8674(00)81683-9.

4. Hanahan D, Weinberg RA. Hallmarks of cancer: The next generation. Cell. 2011;144(5):646-674. doi:10.1016/j.cell.2011.02.013.

5. Ling D, Park W, Park SJ, et al. Multifunctional tumor $\mathrm{pH}$-sensitive self-assembled nanoparticles for bimodal imaging and treatment of resistant heterogeneous tumors. J Am Chem Soc. 2014;136(15):5647-5655. doi:10.1021/ja4108287.

6. Lopci E, Grassi I, Chiti A, et al. PET radiopharmaceuticals for imaging of tumor hypoxia: a review of the evidence. Am J Nucl Med Mol Imaging. 2014;4(4):365-384.

http://www.ncbi.nlm.nih.gov/pubmed/24982822.

7. Gatenby RA, Gillies RJ. Why do cancers have high aerobic glycolysis? Nat Rev Cancer. 2004;4(11):891-899. doi:10.1038/nrc1478.

8. Duncan C, Dougall H, Johnston P, Green S. () 1995 Nature Publishing Group http://www.nature.com/naturemedicine. Nat Med. 1995;1(6):546-551. doi:10.1038/nm0495-365.

9. Iyer AK, Khaled G, Fang J, Maeda H. Exploiting the enhanced permeability and retention effect for tumor targeting. Drug Discov Today. 2006;11(17-18):812-818. doi:10.1016/j.drudis.2006.07.005.

10. Weidner N. New paradigm for vessel intravasation by tumor cells. Am J Pathol. 2002;160(6):1937-1939. doi:10.1016/S0002-9440(10)61141-8.

11. Balkwill FR, Capasso M, Hagemann T. The tumor microenvironment at a glance. J Cell Sci. 2012;125(23):5591-5596. doi:10.1242/jcs.116392. 
12. Milne K, Köbel M, Kalloger SE, et al. Systematic analysis of immune infiltrates in high-grade serous ovarian cancer reveals $\mathrm{CD} 20$, FoxP3 and TIA-1 as positive prognostic factors. PLoS One. 2009;4(7). doi:10.1371/journal.pone.0006412.

13. Fridman WH, Pagès F, Sautès-Fridman C, Galon J. The immune contexture in human tumours: impact on clinical outcome. Nat Rev Cancer. 2012;12(4):298-306. doi:10.1038/nrc3245.

14. Condeelis J, Pollard JW. Macrophages: Obligate partners for tumor cell migration, invasion, and metastasis. Cell. 2006;124(2):263-266. doi:10.1016/j.cell.2006.01.007.

15. Nieman KM, Kenny HA, Penicka C V, et al. Adipocytes promote ovarian cancer metastasis and provide energy for rapid tumor growth. Nat Med. 2011;17(11):1498-1503. doi:10.1038/nm.2492.

16. Diegelmann RF, Evans MC. Wound healing: an overview of acute, fibrotic and delayed healing. Front Biosci. 2004;9(4):283-289. doi:10.2741/1184.

17. Hussain SP, Hofseth LJ, Harris CC. Radical causes of cancer. Nat Rev Cancer. 2003;3(4):276-285. doi:10.1038/nrc1046.

18. Schäfer M, Werner S. Cancer as an overhealing wound: an old hypothesis revisited. Nat Rev Mol Cell Biol. 2008;9(8):628-638. doi:10.1038/nrm2455.

19. Erez N, Truitt M, Olson P, Hanahan D. Cancer-Associated Fibroblasts Are Activated in Incipient Neoplasia to Orchestrate Tumor-Promoting Inflammation in an NF-??B-Dependent Manner. Cancer Cell. 2010;17(2):135-147. doi:10.1016/j.ccr.2009.12.041.

20. LeBedis C, Chen K, Fallavollita L, Boutros T, Brodt P. Peripheral lymph node stromal cells can promote growth and tumorigenicity of breast carcinoma cells through the release of IGF-I and EGF. Int J Cancer. 2002;100(1):2-8. doi:10.1002/ijc.10481.

21. Lu P, Weaver VM, Werb Z. The extracellular matrix: A dynamic niche in cancer progression. $J$ Cell Biol. 2012;196(4):395-406. doi:10.1083/jcb.201102147.

22. Lu P, Takai K. Extracellular matrix degradation and remodeling in development and disease. Cold Spring Harb .... 2011:1-24. doi:10.1101/cshperspect.a005058.

23. Hynes RO. The Extracellular Matrix : Science (80- ). 2012;1216(2009). 
doi:10.1126/science.1176009.

24. Delpech B, Maingonnat C, Girard N, et al. Hyaluronan and hyaluronectin in the extracellular matrix of human brain tumour stroma. Eur J Cancer. 1993;29A(7):1012-1017.

25. Aitken KJ, Bägli DJ. The bladder extracellular matrix. Part II: regenerative applications. Nat Rev Urol. 2009;6(11):612-621. doi:10.1038/nrurol.2009.202.

26. Frantz C, Stewart KM, Weaver VM. The extracellular matrix at a glance. J Cell Sci. 2010;123:4195-4200. doi:10.1242/jcs.023820.

27. Kessenbrock K, Plaks V, Werb Z. Matrix Metalloproteinases: Regulators of the Tumor Microenvironment. Cell. 2010;141(1):52-67. doi:10.1016/j.cell.2010.03.015.

28. Yu Q, Toole B, Stamenkovic I. Induction of apoptosis of metastatic mammary carcinoma cells in vivo by disruption of tumor cell surface CD44 function. J Exp Med. 1997;186(12):1985-1996. doi:10.1084/jem.186.12.1985.

29. Jain RK, Tong RT, Munn LL. Effect of vascular normalization by antiangiogenic therapy on interstitial hypertension, peritumor edema, and lymphatic metastasis: Insights from a mathematical model. Cancer Res. 2007;67(6):2729-2735. doi:10.1158/0008-5472.CAN-06-4102.

30. Jain RK, Baxter LT. Mechanisms of heterogeneous distribution of monoclonal antibodies and other macromolecules in tumors: Significance of elevated interstitial pressure. Cancer Res. 1988;48(18):7022-7032.

31. Minchinton AI, Tannock IF. Drug penetration in solid tumours. Nat Rev Cancer. 2006;6(8):583592. doi:10.1038/nrc1893.

32. Jang SH, Wientjes MG, Lu D, Au JLS. Drug delivery and transport to solid tumors. Pharm Res. 2003;20(9):1337-1350. doi:10.1023/A:1025785505977.

33. Welter M, Rieger H. Interstitial Fluid Flow and Drug Delivery in Vascularized Tumors: A Computational Model. PLoS One. 2013;8(8). doi:10.1371/journal.pone.0070395.

34. Jain RK. Transport of Molecules in the Tumor Interstitium : A Review. Cancer Res. 1987;47(17):3039-3051. doi:10.1007/BF00047468. 
35. Jain RK. Transport of Molecules, Particles, and Cells in Solid Tumors. Annu Rev Biomed Eng. 1999;1(96):241-263. doi:10.1146/annurev.bioeng.1.1.241.

36. Pickup MW, Mouw JK, Weaver VM. The extracellular matrix modulates the hallmarks of cancer. EMBO Rep. 2014;15(12):1243-1253. doi:10.15252/embr.201439246.

37. Lankelma J, Dekker H, Luque RF, Luykx S, Hoekman K, Valk P Van Der. Doxorubicin Gradients in Human Breast Cancer Doxorubicin Gradients in Human Breast Cancer. 1999;5(July):17031707.

38. Gottesman MM. M c d r. 2002.

39. Austin Doyle L, Ross DD. Multidrug resistance mediated by the breast cancer resistance protein BCRP (ABCG2). Oncogene. 2003;22(47):7340-7358. doi:10.1038/sj.onc.1206938.

40. Gottesman MM, Fojo T, Bates SE. Multidrug Resistance in Cancer: Role of Atp-Dependent Transporters. Nat Rev Cancer. 2002;2(1):48-58. doi:10.1038/nrc706.

41. Shen DW, Goldenberg S, Pastan I, Gottesman MM. Decreased accumulation of [C-14]carboplatin in human cisplatin-resistant cells results from reduced energy-dependent uptake. J Cell Physiol. 2000;183(1):108-116. doi:10.1002/(SICI)1097-4652(200004)183:1<108::AID-JCP13>3.0.CO;2-4.

42. Marusyk A, Tabassum DP, Janiszewska M, et al. Spatial proximity to fibroblasts impacts molecular features and therapeutic sensitivity of breast cancer cells influencing clinical outcomes. Cancer Res. 2016;76(22):6495-6506. doi:10.1158/0008-5472.CAN-16-1457.

43. Farmer P, Bonnefoi $\mathrm{H}$, Anderle $\mathrm{P}$, et al. A stroma-related gene signature predicts resistance to neoadjuvant chemotherapy in breast cancer. Nat Med. 2009;15(1):68-74. http://dx.doi.org/10.1038/nm.1908.

44. Loeffler M, Krüger JA, Niethammer AG, Reisfeld RA. Targeting tumor-associated fibroblasts improves cancer chemotherapy by increasing intratumoral drug uptake. J Clin Invest. 2006;116(7):1955-1962. doi:10.1172/JCI26532.

45. Yilmaz M, Christofori G. Mechanisms of motility in metastasizing cells. Mol Cancer Res. 2010;8(5):629-642. doi:10.1158/1541-7786.MCR-10-0139. 
46. Chalovich JM, Eisenberg E. NIH Public Access. Biophys Chem. 2005;257(5):2432-2437. doi:10.1016/j.immuni.2010.12.017.Two-stage.

47. Balkwill F. Chemokine biology in cancer. Semin Immunol. 2003;15(1):49-55. doi:10.1016/S10445323(02)00127-6.

48. $\mathrm{Hu} \mathrm{L}$, Lau SH, Tzang C-H, et al. Association of Vimentin overexpression and hepatocellular carcinoma metastasis. Oncogene. 2004;23(1):298-302. doi:10.1038/sj.onc.1206483.

49. Nabeshima K, Inoue T, Shimao Y, Sameshima T. Matrix metalloproteinases in tumor invasion: Role for cell migration. Pathol Int. 2002;52(4):255-264. doi:10.1046/j.1440-1827.2002.01343.x.

50. Brakebusch C, Bouvard D, Stanchi F, Sakai T, Fässler R. Integrins in invasive growth. J Clin Invest. 2002;109(8):999-1006. doi:10.1172/JCI200215468.

51. Ponta H, Sherman L, Herrlich P a. CD44: from adhesion molecules to signalling regulators. Nat Rev Mol Cell Biol. 2003;4(1):33-45. doi:10.1038/nrm1004.

52. Sahai E, Marshall CJ. Rho-Gtpases and Cancer. Nat Rev Cancer. 2002;2(2):133-142. doi:10.1038/nrc725.

53. Haessler U, Teo JCM, Foretay D, Renaud P, Swartz M a. Migration dynamics of breast cancer cells in a tunable 3D interstitial flow chamber. Integr Biol. 2012;4(4):401. doi:10.1039/c1ib00128k.

54. Kingsmore KM, Logsdon DK, Floyd DH, et al. Interstitial flow differentially increases patientderived glioblastoma stem cell invasion via CXCR4, CXCL12, and CD44-mediated mechanisms. Integr Biol. 2016;4:127ra36. doi:10.1039/C6IB00167J.

55. Munson JM, Bellamkonda R V., Swartz MA. Interstitial flow in a 3d microenvironment increases glioma invasion by a cxcr4-dependent mechanism. Cancer Res. 2013;73(5):1536-1546. doi:10.1158/0008-5472.CAN-12-2838.

56. Qazi H, Palomino R, Shi Z-D, Munn LL, Tarbell JM. Cancer Cell Glycocalyx Mediates Mechanostransduction and Flow-Regulated Invasion. Integr Biol. 2013;5(11):1334-1343. doi:10.1039/c3ib40057c. 
57. Orian-Rousseau V. CD44 Acts as a Signaling Platform Controlling Tumor Progression and Metastasis. Front Immunol. 2015;6(April):154. doi:10.3389/fimmu.2015.00154.

58. Sung KE, Su X, Berthier E, Pehlke C, Friedl A, Beebe DJ. Understanding the Impact of 2D and 3D Fibroblast Cultures on In Vitro Breast Cancer Models. PLoS One. 2013;8(10):1-14. doi:10.1371/journal.pone.0076373.

59. Hsiao AY, Torisawa Y suke, Tung YC, et al. Microfluidic system for formation of PC-3 prostate cancer co-culture spheroids. Biomaterials. 2009;30(16):3020-3027. doi:10.1016/j.biomaterials.2009.02.047.

60. Zanoni M, Piccinini F, Arienti C, et al. 3D tumor spheroid models for in vitro therapeutic screening: a systematic approach to enhance the biological relevance of data obtained. Sci Rep. 2016;6(August 2015):19103. doi:10.1038/srep19103.

61. Mehta G, Hsiao AY, Ingram M, Luker GD, Takayama S. Opportunities and challenges for use of tumor spheroids as models to test drug delivery and efficacy. J Control Release. 2012;164(2):192204. doi:10.1016/j.jconrel.2012.04.045.

62. Nyga A, Cheema U, Loizidou M. 3D tumour models: Novel in vitro approaches to cancer studies. J Cell Commun Signal. 2011;5(3):239-248. doi:10.1007/s12079-011-0132-4.

63. Gurski LA, Jha AK, Zhang C, Jia X, Farach-Carson MC. Hyaluronic acid-based hydrogels as 3D matrices for in vitro evaluation of chemotherapeutic drugs using poorly adherent prostate cancer cells. Biomaterials. 2009;30(30):6076-6085. doi:10.1016/j.biomaterials.2009.07.054.

64. Yuan JX, Bafakih FF, Mandell JW, Horton BJ, Munson JM. Quantitative Analysis of the Cellular Microenvironment of Glioblastoma to Develop Predictive Statistical Models of Overall Survival. J Neuropathol Exp Neurol. 2016;75(12):1110-1123. doi:10.1093/jnen/nlw090.

65. Walpole J, Chappell JC, Cluceru JG, Mac Gabhann F, Bautch VL, Peirce SM. Agent-based model of angiogenesis simulates capillary sprout initiation in multicellular networks. Integr Biol (Camb). 2015;7(9):987-997. doi:10.1039/c5ib00024f.

66. Martin KS, Blemker SS, Peirce SM. Agent-based computational model investigates muscle- 
specific responses to disuse-induced atrophy. J Appl Physiol. 2015;118(10):1299-1309. doi:10.1152/japplphysiol.01150.2014.

67. Emonet T, Macal CM, North MJ, Wickersham CE, Cluzel P. AgentCell: A digital single-cell assay for bacterial chemotaxis. Bioinformatics. 2005;21(11):2714-2721. doi:10.1093/bioinformatics/bti391.

68. Enderling H, Hlatky L, Hahnfeldt P. Migration rules: tumours are conglomerates of selfmetastases. Br J Cancer. 2009;100(12):1917-1925. doi:10.1038/sj.bjc.6605071.

69. Enderling H, Hlatky L, Hahnfeldt P. Tumor morphological evolution: directed migration and gain and loss of the self-metastatic phenotype. Biol Direct. 2010;5:23. doi:10.1186/1745-6150-5-23.

70. Cosgrove J, Butler J, Alden K, et al. Agent-based modeling in systems pharmacology. CPT Pharmacometrics Syst Pharmacol. 2015;4(11):615-629. doi:10.1002/psp4.12018.

71. Barros de Andrade e Sousa LC, Kühn C, Tyc KM, Klipp E. Dosage and dose schedule screening of drug combinations in agent-based models reveals hidden synergies. Front Physiol. 2016;6(JAN):1-12. doi:10.3389/fphys.2015.00398.

72. Kazmi N, Hossain MA, Phillips RM. A hybrid cellular automaton model of solid tumor growth and bioreductive drug transport. IEEE/ACM Trans Comput Biol Bioinform. 2012;9(6):1595-1606. doi:10.1109/TCBB.2012.118.

73. Giese A, Westphal M. Glioma invasion in the central nervous system. Neurosurgery. 1996;39(2):235-50-2.

74. Bellail AC, Hunter SB, Brat DJ, Tan C, Van Meir EG. Microregional extracellular matrix heterogeneity in brain modulates glioma cell invasion. Int J Biochem Cell Biol. 2004;36(6):10461069. doi:10.1016/j.biocel.2004.01.013.

75. Boucher Y, Salehil H, Witwerl B, Harsh G, Jain R. Interstitial fluid pressure in intracranial tumours in patients and in rodents. Br Joumal Cancer. 1997;75(6):829-836.

76. Munson JM, Shieh AC. Interstitial fluid flow in cancer: implications for disease progression and treatment. Cancer Manag Res. 2014;6:317-328. doi:10.2147/CMAR.S65444. 
77. Lee J, Kotliarova S, Kotliarov Y, et al. Tumor stem cells derived from glioblastomas cultured in bFGF and EGF more closely mirror the phenotype and genotype of primary tumors than do serumcultured cell lines. Cancer Cell. 2006;9(5):391-403. doi:10.1016/j.ccr.2006.03.030.

78. Geer CP, Grossman SA. Interstitial fluid flow along white matter tracts: a potentially important mechanism for the dissemination of primary brain tumors. J Neurooncol. 1997;32(3):193-201.

79. Shah AD, Bouchard MJ, Shieh AC. Interstitial Fluid Flow Increases Hepatocellular Carcinoma Cell Invasion through CXCR4/CXCL12 and MEK/ERK Signaling. PLoS One. 2015;10(11):1-17. doi:10.1371/journal.pone.0142337.

80. Adler J, Dahl MM. A method for measuring the motility of bacteria and for comparing random and non-random motility. J Gen Microbiol. 1967;46(2):161-173. doi:10.1099/00221287-46-2-161.

81. Radenovic A. Brownian Motion and Single Particle Tracking. LbenEpflCh. 2005:1-23. http://lben.epfl.ch/files/content/sites/lben/files/users/179705/Brownian Motion Handout.pdf.

82. Sikov WM, Berry DA, Perou CM, et al. Impact of the addition of carboplatin and/or bevacizumab to neoadjuvant once-per-week paclitaxel followed by dose-dense doxorubicin and cyclophosphamide on pathologic complete response rates in stage II to III triple-negative breast cancer: CALGB 40603 (A. J Clin Oncol. 2015;33(1):13-21. doi:10.1200/JCO.2014.57.0572.

83. Kim H-J, Im S-A, Keam B, et al. ABCB1 polymorphism as prognostic factor in breast cancer patients treated with docetaxel and doxorubicin neoadjuvant chemotherapy. Cancer Sci. 2015;106(1):86-93. doi:10.1111/cas.12560.

84. Carey LA, Dees EC, Sawyer L, et al. The triple negative paradox: Primary tumor chemosensitivity of breast cancer subtypes. Clin Cancer Res. 2007;13(8):2329-2334. doi:10.1158/1078-0432.CCR06-1109.

85. Heldin C-H, Rubin K, Pietras K, Ostman A. High interstitial fluid pressure - an obstacle in cancer therapy. Nat Rev Cancer. 2004;4(10):806-813. doi:10.1038/nrc1456.

86. Trédan O, Galmarini CM, Patel K, Tannock IF. Drug Resistance and the Solid Tumor Microenvironment. JNCI J Natl Cancer Inst. 2007;99(19):1441-1454. 
http://dx.doi.org/10.1093/jnci/djm135.

87. Shannon AM, Bouchier-Hayes DJ, Condron CM, Toomey D. Tumour hypoxia, chemotherapeutic resistance and hypoxia-related therapies. Cancer Treat Rev. 2003;29(4):297-307. doi:10.1016/S0305-7372(03)00003-3.

88. Raghunand N, He X, van Sluis R, et al. Enhancement of chemotherapy by manipulation of tumour pH. Br J Cancer. 1999;80:1005-1011. doi:10.1038/sj.bjc.6690455.

89. Ljungkvist ASE, Bussink J, Rijken PFJW, Kaanders JHAM, Van der Kogel AJ, Denekamp J. Vascular architecture, hypoxia, and proliferation in first-generation xenografts of human headand-neck squamous cell carcinomas. Int J Radiat Oncol Biol Phys. 2002;54(1):215-228. doi:10.1016/S0360-3016(02)02938-3.

90. Shain KH, Dalton WS. Cell adhesion is a key determinant in de novo multidrug resistance (MDR): new targets for the prevention of acquired MDR. Mol Cancer Ther. 2001;1(1):69-78. http://www.ncbi.nlm.nih.gov/pubmed/12467240.

91. Hazlehurst L a, Damiano JS, Buyuksal I, Pledger WJ, Dalton WS. Adhesion to fibronectin via beta1 integrins regulates p27kip1 levels and contributes to cell adhesion mediated drug resistance (CAM-DR). Oncogene. 2000;19:4319-4327. doi:10.1038/sj.onc.1203782.

92. Pietras K, Östman A. Hallmarks of cancer: Interactions with the tumor stroma. Exp Cell Res. 2010;316(8):1324-1331. doi:10.1016/j.yexcr.2010.02.045.

93. Gao M-Q, Kim BG, Kang S, et al. Stromal fibroblasts from the interface zone of human breast carcinomas induce an epithelial-mesenchymal transition-like state in breast cancer cells in vitro. $J$ Cell Sci. 2010;123(Pt 20):3507-3514. doi:10.1242/jcs.072900.

94. Griffith LG, Swartz MA. Capturing complex 3D tissue physiology in vitro. Nat Rev Mol cell Biol. 2006;7(3):211-224. doi:10.1038/nrm1858.

95. Ramanujan S, Pluen A, McKee TD, Brown EB, Boucher Y, Jain RK. Diffusion and convection in collagen gels: implications for transport in the tumor interstitium. Biophys J. 2002;83(3):16501660. doi:10.1016/S0006-3495(02)73933-7. 
96. Ueda K, Cardarelli C, Gottesman MM, Pastan I. Expression of a full-length cDNA for the human "MDR1" gene confers resistance to colchicine, doxorubicin, and vinblastine. Proc Natl Acad Sci U S A. 1987;84(9):3004-3008. doi:10.1073/pnas.84.9.3004.

97. Shen F, Chu S, Bence AK, et al. Quantitation of Doxorubicin Uptake, Efflux , and Modulation of Multidrug Resistance ( MDR ) in MDR Human Cancer Cells. 2008;324(1):95-102. doi:10.1124/jpet.107.127704.with.

98. Wong HL, Bendayan R, Rauth AM, Xue HY, Babakhanian K. A Mechanistic Study of Enhanced Doxorubicin Uptake and Retention in Multidrug Resistant Breast Cancer Cells Using a PolymerLipid Hybrid Nanoparticle System. 2006;317(3):1372-1381. doi:10.1124/jpet.106.101154.drug.

99. Lankelma J, Dekker H, Luykx S, et al. Doxorubicin Gradients in Human Breast Cancer. Clin Cancer Res. 1999;5(July):1703-1707.

100. Kalluri R, Zeisberg M. Fibroblasts in cancer. Nat Rev Cancer. 2006;6(5):392-401. doi:10.1038/nrc1877.

101. Cells C, Conze D, Weiss L, et al. Autocrine Production of Interleukin 6 Causes Multidrug Resistance in Breast. 2001:8851-8858.

102. Bandyopadhyay A, Wang L, Agyin J, et al. Doxorubicin in Combination with a Small TGF b Inhibitor: A Potential Novel Therapy for Metastatic Breast Cancer in Mouse Models. 2010;5(4). doi:10.1371/journal.pone.0010365.

103. Sethi T, Rintoul RC, Moore SM, et al. Extracellular matrix proteins protect small cell lung cancer cells against apoptosis: a mechanism for small cell lung cancer growth and drug resistance in vivo. Nat Med. 1999;5(6):662-668. doi:10.1038/9511.

104. Fang W Bin, Yao M, Cheng N. Priming cancer cells for drug resistance: role of the fibroblast niche. Front Biolofy. 2014;9(2):114-126. doi:10.1007/s11515-014-1300-8.Priming.

105. Huber S, Delorme S, Knopp M V, et al. Breast tumors: computer-assisted quantitative assessment with color Doppler US. Radiology. 1994;192(3):797-801. doi:10.1148/radiology.192.3.8058950.

106. Navalitloha Y, Schwartz ES, Groothuis EN, Allen C V, Levy RM, Groothuis DR. Therapeutic 
implications of tumor interstitial fluid pressure in subcutaneous RG-2 tumors. Neuro Oncol. 2006;8(3):227-233. doi:10.1215/15228517-2006-007.

107. Munson JM, Fried L, Rowson S a, et al. Anti-invasive adjuvant therapy with imipramine blue enhances chemotherapeutic efficacy against glioma. Sci Transl Med. 2012;4(127):127ra36. doi:10.1126/scitranslmed.3003016.

108. Gritsenko PG, Ilina O, Friedl P. Interstitial guidance of cancer invasion. J Pathol. 2012;226(Table 1):185-199. doi:10.1002/path.3031.

109. Tchou J, Conejo-Garcia J. Targeting the Tumor Stroma as a Novel Treatment Strategy for Breast Cancer: Shifting from the Neoplastic Cell-Centric to a Stroma-Centric Paradigm. Vol 65. Elsevier Inc.; 2012. doi:10.1016/B978-0-12-397927-8.00003-8.

110. Friedl P, Alexander S. Cancer invasion and the microenvironment: plasticity and reciprocity. Cell. 2011;147(5):992-1009. doi:10.1016/j.cell.2011.11.016.

111. Provenzano PP, Cuevas C, Chang AE, Goel VK, Von Hoff DD, Hingorani SR. Enzymatic targeting of the stroma ablates physical barriers to treatment of pancreatic ductal adenocarcinoma. Cancer Cell. 2012;21(3):418-429. doi:10.1016/j.ccr.2012.01.007.

112. Provenzano PP, Hingorani SR. Hyaluronan, fluid pressure, and stromal resistance in pancreas cancer. Br J Cancer. 2013;108(1):1-8. doi:10.1038/bjc.2012.569.

113. Shieh AC, Rozansky HA, Hinz B, Swartz MA. Tumor cell invasion is promoted by interstitial flow-induced matrix priming by stromal fibroblasts. Cancer Res. 2011;71(3):790-800. doi:10.1158/0008-5472.CAN-10-1513.

114. Stupp R, Hegi ME, Mason WP, et al. Effects of radiotherapy with concomitant and adjuvant temozolomide versus radiotherapy alone on survival in glioblastoma in a randomised phase III study: 5-year analysis of the EORTC-NCIC trial. Lancet Oncol. 2009;10(5):459-466. doi:10.1016/S1470-2045(09)70025-7. 
